\title{
Dataset of noncovalent intermolecular interaction energy curves for 24 small high-spin open-shell dimers
}

Katarzyna Madajczyk, ${ }^{1}$ Piotr S. Żuchowski, ${ }^{1,}$ a) Filip Brzęk, ${ }^{1}$ Łukasz Rajchel, ${ }^{2}$ Dariusz Kędziera, ${ }^{3}$ Marcin Modrzejewski, ${ }^{4}$ and Michał Hapka ${ }^{4,5, b)}$

1) Institute of Physics, Faculty of Physics, Astronomy and Informatics, Nicolaus Copernicus University, Grudziadzka 5, 87-100, Toruń,

Poland

2) Institute of Physics, Kazimierz Wielki University, al. Powstańców Wielkopolskich 2, 85-090 Bydgoszcz, Poland

3) Faculty of Chemistry, Nicolaus Copernicus University, Toruń, Poland

${ }^{4)}$ Faculty of Chemistry, University of Warsaw, ul. L. Pasteura 1, 02-093 Warsaw, Poland

5) Department of Chemistry, University of Michigan, Ann Arbor, Michigan 48109, $U S A$ 
We introduce a dataset of 24 interaction energy curves of open-shell noncovalent dimers, referred to as the $024 \times 5$ dataset. The dataset consists of high-spin dimers up to eleven atoms selected to assure diversity with respect to interactions types: dispersion, electrostatics and induction. The benchmark interaction energies are obtained at the restricted open-shell $\operatorname{CCSD}(\mathrm{T})$ level of theory with complete basis set extrapolation (aug-cc-pVQZ $\longrightarrow$ aug-cc-pV5Z). We have analyzed the performance of selected wave function methods: MP2, CCSD, CCSD(T), as well as the F12a and F12b variants of coupled-cluster theory. In addition, we have tested dispersioncorrected DFT methods based on the PBE exchange-correlation model. The O24x5 dataset is a challenge to approximate methods due to the wide range of the interaction energy strengths it spans. For the dispersion-dominated and mixed-type subsets any tested method that does not include the triples contribution yields errors on the order of tens of percent. The electrostatic subset is less demanding with errors that are typically an order of magnitude smaller than the mixed and dispersion-dominated subsets.

a) Electronic mail: pzuch@fizyka.umk.pl

b) Electronic mail: hapka@tiger.chem.uw.edu.pl 


\section{INTRODUCTION}

Noncovalent interactions involving open-shell atoms, molecules and ions are of great importance in chemistry. ${ }^{1}$ One of the most prominent examples include chemical reactions in which molecules experience the bond breaking and fragmentation into radicals. The spectroscopy of radicals is a basic tool of astrophysics. ${ }^{2}$ Similarly, the information about interactions of open-shell molecules in the atmosphere, in particular of the molecular oxygen, is needed for modelling spectral line shapes or collision-induced absorption that are

used for remote sensing of atmospheric gases. ${ }^{3}$ Open-shell species are also relevant for the cold matter community, as it is now possible to accurately measure the collision properties, such as resonance positions. ${ }^{4,5}$ Ab initio calculations of these properties are used to design experiments in which molecules are cooled by collisions with ultracold atomic gas. ${ }^{6-8}$ Finally, there is a considerable interest in interactions of large open-shell organic compounds ${ }^{9}$ which might be useful for materials science. ${ }^{10,11}$

While there exists demand for low-cost methods applicable to open-shell noncovalent complexes, no systematic dataset has been established to test these methods. Such dataset is necessary to benchmark low-cost approaches before their use in large systems, where applying high-level wave-function approximations in the basis set limit is no longer feasible.

The protocol of testing the performance of quantum chemistry methods for noncovalent systems has changed quite dramatically over the past fifteen years. In particular, a detailed statistical analysis of big, properly balanced, and diverse benchmark data sets of noncovalently bound complexes has been established as a standard tool. ${ }^{12,13}$ In addition to the equilibrium geometries corresponding to van der Waals minima, these sets now typically include geometries at large intermonomer separation and at distances shorter than the van der Waals minimum. Examples of datasets featuring radial diversity are the NBC10 ${ }^{14,15}$ database of Sherrill an co-workers, an extension of the S22 ${ }^{16-18}$ dataset known as S22x5, ${ }^{19}$ or the dataset of Ref. 20. There exist sets which also probe the angular dependence, widelyknown examples being the S66x8 dataset of ŘRezác et al. ${ }^{21}$ and the database of Smith et al. ${ }^{22}$ which also featured reparametrization of the DFT-D3 model.

There exists no open-shell equivalent of the well-established closed-shell noncovalent datasets. ${ }^{13}$ However, several specific-purpose datasets have been reported. Steinmann and Corminboeuf $^{9}$ designed one of the first such sets, Orel26rad, for organic radicals and radical 
cations. Another open-shell set was introduced by Tentscher and Arey. ${ }^{23,24}$ Alday et al. ${ }^{25}$ composed a small set containing $\mathrm{H}_{2} \mathrm{~S}-\mathrm{X}(\mathrm{X}=\mathrm{F}, \mathrm{Cl}, \mathrm{Br}, \mathrm{OH})$ complexes. To test the performance of open-shell MP2 and MP3 methods, Soydas and Bozkaya ${ }^{26}$ composed a set of 23 complexes which included systems from the set of Tentscher and Arey ${ }^{23}$ and dimers studied with open-shell variants of the symmetry-adapted perturbation theory (SAPT). ${ }^{27,28}$

In this work we introduce a new open-shell database of 24 dimers, referred to as $\mathrm{O} 24 \mathrm{x} 5$. The dimers were chosen to cover a wide range of interaction types and are relevant to atmospheric science, astrochemistry and cold chemistry. The selected dimers contain up to 11 atoms. Each complex is represented by a 5-point radial curve, so that the database consists of 120 data points. We restrict ourselves to high-spin systems to exclude the possibility of covalent bond formation and allow for a single-reference description. We also avoid both spin and spatial degeneracy. Limiting the scope to small single-reference systems warrants the use of restricted open-shell, partially spin-adapted coupled-cluster singles-and-doubles with perturbative triples $[\mathrm{RCCSD}(\mathrm{T})]^{29,30}$ results extrapolated to the complete basis set (CBS) limit as the high-quality benchmark.

In addition to providing benchmark interaction energies, we test the accuracy of several approximate approaches which can be efficiently applied to larger open-shell systems: $\operatorname{CCSD}(\mathrm{T})-\mathrm{F} 12 \mathrm{a}$ and $\operatorname{CCSD}(\mathrm{T})-\mathrm{F} 12 \mathrm{~b}$ applied with a triple- $\zeta$ basis set, CCSD, MP2, and dispersion-corrected density functional theory (DFT).

\section{O24x5 DATABASE COMPOSITION}

The $\mathrm{O} 24 \times 5$ dataset includes small molecular systems consisting of light atoms. We have reviewed and selected systems relevant in both experimental and theoretical research:

- dimers containing $\mathrm{O}_{2}, \mathrm{OH}, \mathrm{O}_{2} \mathrm{H}$, which are important in atmospheric research and appear in the transmission molecular absorption database HITRAN ${ }^{31}: \mathrm{H}_{2} \mathrm{O}-\mathrm{OH}$, $\mathrm{H}_{2} \mathrm{O}-\mathrm{O}_{2} \mathrm{H}, \mathrm{H}_{2} \mathrm{O}-\mathrm{O}_{2}, \mathrm{CO}_{2}-\mathrm{O}_{2}, \mathrm{O}_{2}-\mathrm{H}_{2}, \mathrm{O}_{2}-\mathrm{N}_{2}$, and $\mathrm{O}_{2}-\mathrm{O}_{2}$;

- systems which were studied due to their importance in astrochemistry: $\mathrm{CN}-\mathrm{He}$, $\mathrm{NH}-\mathrm{He}, \mathrm{CN}-\mathrm{H}_{2}$ and $\mathrm{O}_{2}-\mathrm{H}_{2}$ from the BASECOL ${ }^{32}$ database and $\mathrm{H}_{2} \mathrm{CO}-\mathrm{NH}_{2}$ studied recently by Barone et al. ${ }^{33}$ as a van der Waals precursor of formamide formation;

- systems important in cold chemistry: $\mathrm{Na}-\mathrm{Li}, \mathrm{He}^{*}-\mathrm{He}^{*}$ in the quintet state, high- 
spin $\mathrm{NH}-\mathrm{NH}, \mathrm{Li}-\mathrm{NH}_{3}, \mathrm{Li}-\mathrm{O}_{2}$ and $\mathrm{Na}-\mathrm{H}_{2} \mathrm{O}$; these dimers include highly polarizable atoms interacting with paramagnetic molecules or molecules with lone electronic pairs;

- open-shell noncovalent systems selected as test cases in several method-development papers: $\mathrm{O}_{2}-\mathrm{O}_{2}, \mathrm{CN}-\mathrm{Ar}$ and $\mathrm{NH}-\mathrm{Ar} ;{ }^{26-28,34,35}$

- two dimers which include the vinyl radical $\left(\mathrm{C}_{2} \mathrm{H}_{3}-\mathrm{C}_{2} \mathrm{H}_{4}, \mathrm{C}_{2} \mathrm{H}_{3}-\mathrm{CO}_{2}\right)$ that serve as a prototype of $\pi-\pi$ interactions;

- to represent charged systems we use cation-radical $\mathrm{CO}^{+}$interacting with the $\mathrm{HF}$ molecule, studied by Soydas and Bozkaya. ${ }^{26}$

Except for $\mathrm{Li}-\mathrm{O}_{2}$ and $\mathrm{C}_{2} \mathrm{H}_{4}-\mathrm{C}_{2} \mathrm{H}_{3}$, we use equilibrium geometries from the literature (see Tables I-III). The $\mathrm{Li}-\mathrm{NH}_{3}$ and $\mathrm{H}_{2} \mathrm{O}-\mathrm{O}_{2}$ complexes are represented by two different geometries each. In $\mathrm{Li}-\mathrm{NH}_{3}$ the lithium atom is located on the $C_{3 v}$ axis either on the nitrogen side (deep global minimum), or on the hydrogen side (shallow secondary minimum). For the water- $\mathrm{O}_{2}$ dimer we include both the global minimum and the saddle point, the latter corresponding to the $C_{2 v}$ geometry. For $\mathrm{Li}-\mathrm{O}_{2}$ the global minimum (T-shape geometry) was found using the RCCSD(T) method and the aug-cc-pVQZ basis set with midbond functions. For the $\mathrm{C}_{2} \mathrm{H}_{4}-\mathrm{C}_{2} \mathrm{H}_{3}$ dimer we selected the geometry in which the symmetry planes $\left(C_{s}\right)$ of both molecules are parallel and double bonds are aligned. The minimum energy geometry for this configuration was optimized at the RCCSD $(T)$ level of theory with the aug-cc-pVTZ basis set with midbond functions. Note that the vinyl radical in $\mathrm{C}_{2} \mathrm{H}_{4}-\mathrm{C}_{2} \mathrm{H}_{3}$ is not in the equilibrium geometry. Finally, it should be mentioned that the $\mathrm{H}_{2} \mathrm{O}-\mathrm{OH}$ dimer is kept in the $C_{s}$ symmetry ( $\mathrm{OH}$ lies in the symmetry plane of the water molecule, see Table II). In this configuration the orbital degeneracy of the $\mathrm{OH}$ molecule is lifted and the corresponding states of the dimer are decoupled, as they can be assigned either to the $A^{\prime}$ or to $A^{\prime \prime}$ symmetry (the latter is lower in energy).

For each complex we consider the separations of $0.9,1.0,1.2,1.5$ and 2.0 times the equilibrium distance $\left(R_{\text {eq }}\right)$ between the centers of masses of the monomers.

The molecule-molecule systems at $R=2 R_{\text {eq }}$ can be classified according to the dominant multipole-multipole contributions to the electrostatic interaction energy:

- strong ion-dipole attraction: $\mathrm{HF}-\mathrm{CO}^{+}$; 
- dipole-dipole attraction: $\mathrm{NH}-\mathrm{NH}, \mathrm{H}_{2} \mathrm{O}-\mathrm{OH}, \mathrm{H}_{2} \mathrm{O}-\mathrm{O}_{2} \mathrm{H}, \mathrm{CH}_{2} \mathrm{O}-\mathrm{NH}_{2}$;

- dipole-quadrupole or quadrupole-quadrupole repulsion: $\mathrm{CN}-\mathrm{H}_{2}, \mathrm{O}_{2}-\mathrm{N}_{2}, \mathrm{O}_{2}-\mathrm{O}_{2}$, $\mathrm{C}_{2} \mathrm{H}_{3}-\mathrm{C}_{2} \mathrm{H}_{4}$, and saddle-point $\mathrm{H}_{2} \mathrm{O}-\mathrm{O}_{2}$; in these cases strong cancellation between first- and second-order interaction energy components occurs; in particular, for both $\mathrm{H}_{2} \mathrm{O}-\mathrm{O}_{2}$ and $\mathrm{C}_{2} \mathrm{H}_{3}-\mathrm{C}_{2} \mathrm{H}_{4}$ the net interaction energy is sensitive to the intramonomer correlation;

- dipole-quadrupole or quadrupole-quadrupole attraction: $\mathrm{C}_{2} \mathrm{H}_{3}-\mathrm{CO}_{2}, \mathrm{H}_{2} \mathrm{O}-\mathrm{O}_{2}$ in the global minimum and $\mathrm{O}_{2}-\mathrm{H}_{2}$.

The systems of the $024 \times 5$ database were chosen to cover the ternary diagram ${ }^{36,37}$ of the interaction energy components as evenly as possible. The classification of systems into dispersion-bound, electrostatic-bound, and mixed dimers was done according to the SAPT interaction energy decomposition. Calculations were performed at equilibrium geometries with the spin-unrestricted SAPT0 method. ${ }^{27,38}$ Tables I-III contain the O24x5 systems grouped into dispersion, electrostatic, and mixed subsets.

In Figure 1 we show the data points of the $024 \times 5$ database in a ternary diagram of intermolecular interactions. The data points other than van der Waals minima cover the dispersion- and electrostatic-dominated types of interaction. Note that it is practically impossible to cover pure induction-dominated systems having no dispersion, because both of those intermolecular forces depend on the polarizability. (Large induction with no accompanying dispersion is however possible, e.g., in complexes with $\mathrm{H}^{+}$ions.) The data points of the $\mathrm{O} 24 \mathrm{x} 5$ dataset probe the ternary diagram to a similar extent as in the well-established closed-shell datasets of a similar size, e.g., the S22+ database ${ }^{60}$ analyzed in Ref. 22.

\section{TECHNICAL DETAILS}

The following wave function calculations were performed in the MOLPRO ${ }^{61}$ package: (i) spin-restricted, partially spin-adapted coupled cluster [referred to as $\operatorname{RCCSD}(\mathrm{T})$ ], as formulated by Knowles et al. ${ }^{29,30}$ (which served as benchmark); (ii) UCCSD/UCCSD(T) ${ }^{62}$ [based on ROHF reference, see Section IVA] (iii) explicitly correlated variants of both RCCSD and UCCSD in the F12a and F12b approximations; ${ }^{63}$ (iv) both spin-restricted and spin-unrestricted MP2. For three dimers $\left(\mathrm{NH}-\mathrm{NH}, \mathrm{Li}-\mathrm{O}_{2}\right.$, and $\left.\mathrm{O}_{2}-\mathrm{O}_{2}\right)$ interaction 
TABLE I. Dispersion subset of the O24x5 dataset. $S_{\mathrm{A}}$ and $S_{\mathrm{B}}$ denote spin multiplicity of monomers $\mathrm{A}$ and $\mathrm{B}$, respectively.

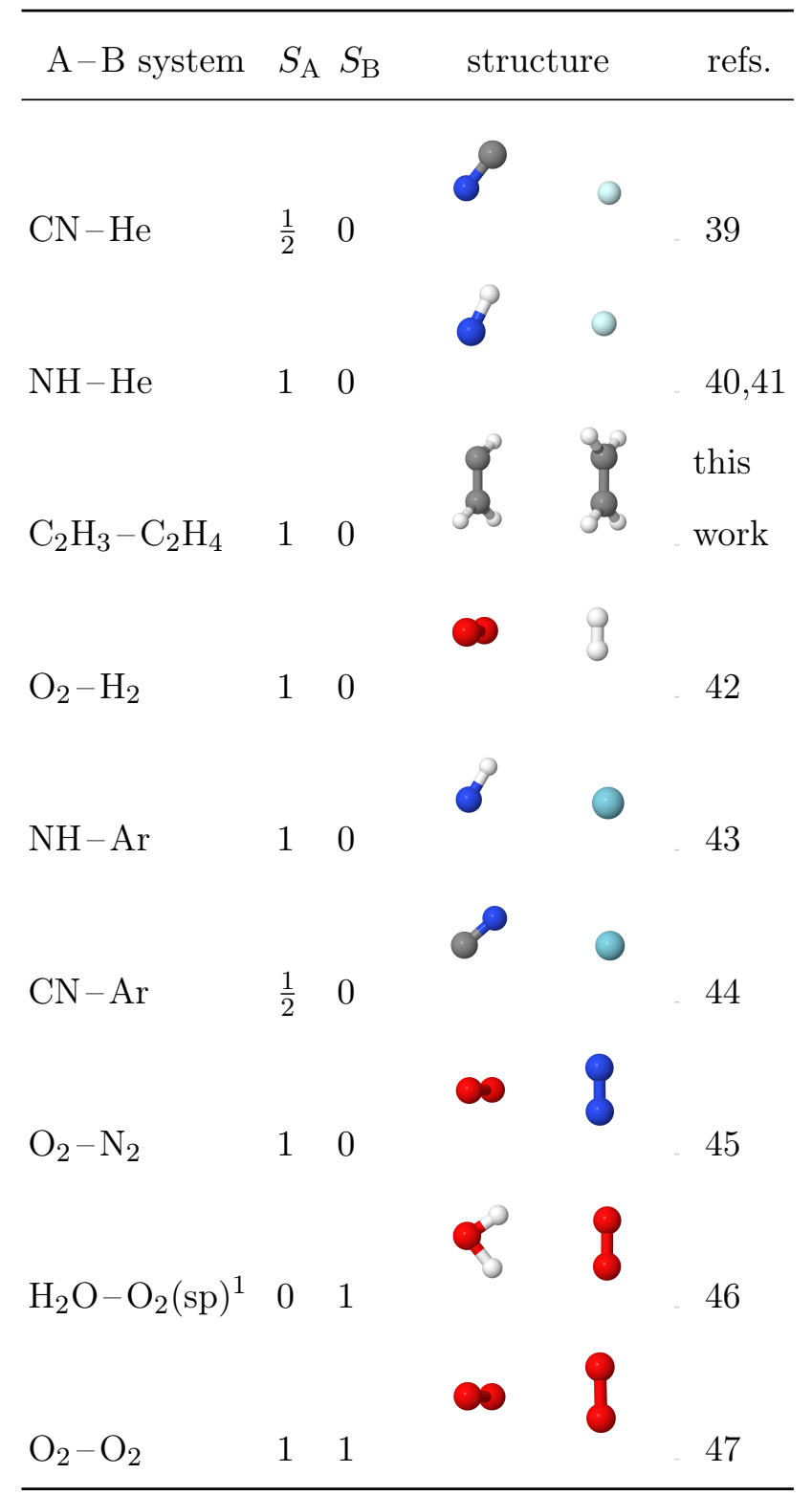

${ }^{1} \mathrm{sp}$ - saddle point

energies from the partially spin-adapted coupled-cluster methods (including F12 variants) were corrected for size-consistency (see Section IV A).

The perturbative triples correction in F12 calculations was scaled by the ratio of MP2 correlation energies ${ }^{63,64}$ according to the formula:

$$
E_{\text {corr }}^{\left(\mathrm{T}^{* *}\right)}=E_{\text {corr }}^{(\mathrm{T})} \times \frac{E_{\text {corr }}^{\mathrm{MP} 2-\mathrm{F} 12}}{E_{\text {corr }}^{\mathrm{MP} 2}}
$$

To warrant size-consistency, the MP2 scaling factor is computed for the dimer and used in 
TABLE II. Electrostatic subset of the O24x5 dataset. $S_{\mathrm{A}}$ and $S_{\mathrm{B}}$ denote spin multiplicity of monomers $\mathrm{A}$ and $\mathrm{B}$, respectively.

\begin{tabular}{|c|c|c|c|c|}
\hline A-B system & $S_{\mathrm{A}}$ & $S_{\mathrm{B}}$ & structure & refs. \\
\hline $\mathrm{NH}-\mathrm{NH}$ & 1 & 1 & & 48 \\
\hline $\mathrm{CH}_{2} \mathrm{O}-\mathrm{NH}_{2}$ & 0 & $\frac{1}{2}$ & & 33 \\
\hline $\mathrm{H}_{2} \mathrm{O}-\mathrm{Na}$ & 0 & $\frac{1}{2}$ & & 49 \\
\hline $\mathrm{H}_{2} \mathrm{O}-\mathrm{OH}$ & 0 & $\frac{1}{2}$ & & 50 \\
\hline $\mathrm{H}_{2} \mathrm{O}-\mathrm{O}_{2} \mathrm{H}$ & 0 & $\frac{1}{2}$ & & 51 \\
\hline $\mathrm{Li}-\mathrm{NH}_{3}(\mathrm{gm})^{1}$ & $\frac{1}{2}$ & 0 & & 7 \\
\hline
\end{tabular}

${ }^{1} \mathrm{gm}$ - global minimum

monomer calculations, which is referred to as the $\mathrm{T}^{* *}$ approach. ${ }^{63,64}$

The spin-unrestricted DFT calculations based on the PBE ${ }^{65} \mathrm{PBE} 0,{ }^{66}$ and LC-VV10 67 exchange-correlation functionals were carried out with the Q-CHEM ${ }^{68}$ package. The QCHEM calculations employed a molecular grid composed of 250 radial points and 1454 angular points. Both LC- $\omega \mathrm{PBE}^{69}$ and LC- $\omega \mathrm{PBE}-\mathrm{D} 3^{70}$ results were obtained with the Gaussian ${ }^{71}$ program with the UltraFine grid settings. All DFT-D3 calculations employed the default dispersion coefficients for a given exchange-correlation functional. The XDM dispersion correction was calculated with the postg ${ }^{72,73}$ program. Spin-unrestricted SAPT0 ${ }^{28,38}$ results were obtained with the PSI4 ${ }^{74}$ package, using the aug-cc-pVTZ basis set with midbond functions. Midbond functions in both SAPT0 calculations and geometry optimization were placed at the dimer's geometric center and used the exponents from the hydrogen basis.

All orbital MP2, UMP2, RCCSD and $\operatorname{RCCSD}(\mathrm{T})$ wave function calculations used the 
TABLE III. Mixed subset of the $\mathrm{O} 24 \times 5$ dataset. $S_{\mathrm{A}}$ and $S_{\mathrm{B}}$ denote spin multiplicity of monomers $\mathrm{A}$ and $\mathrm{B}$, respectively.

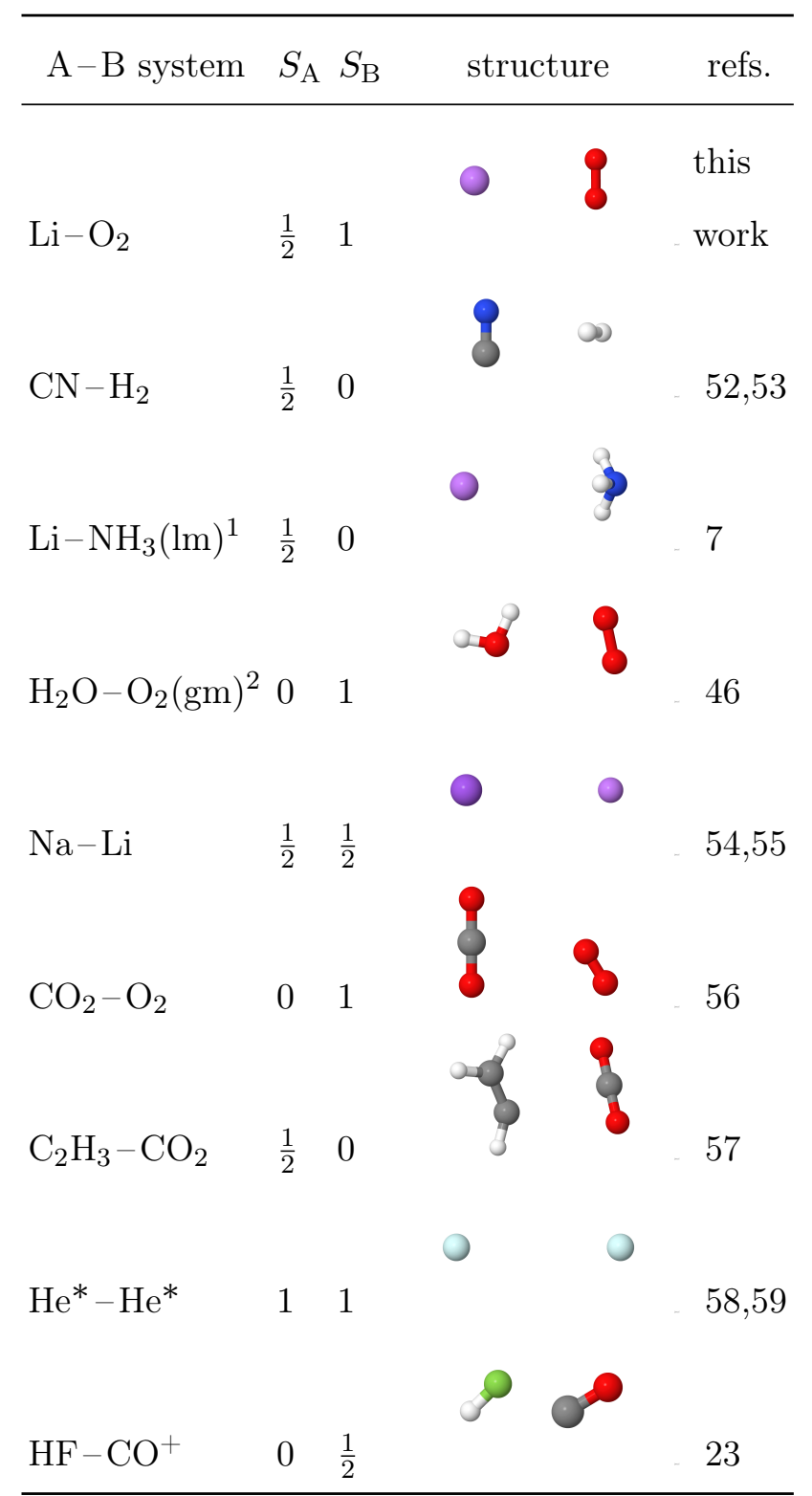

${ }^{1} \mathrm{~lm}$ - local minimum

2 gm - global minimum

complete basis set (CBS) extrapolation formula ${ }^{75,76}$

$$
E_{\mathrm{corr}, \mathrm{CBS}}=\frac{X^{3} E_{\mathrm{corr}, \mathrm{X}}-(X-1)^{3} E_{\mathrm{corr}, \mathrm{X}-1}}{X^{3}-(X-1)^{3}}
$$

where $X$ denotes the cardinal number of the basis set. The extrapolation formula was applied with the correlation consistent aug-cc-pVQZ and aug-cc-pV5Z basis sets ${ }^{77,78}$ in all cases, except for the $\mathrm{He}^{*}-\mathrm{He}^{*}$ dimer where the doubly augmented d-aug-cc-pVQZ and d- 

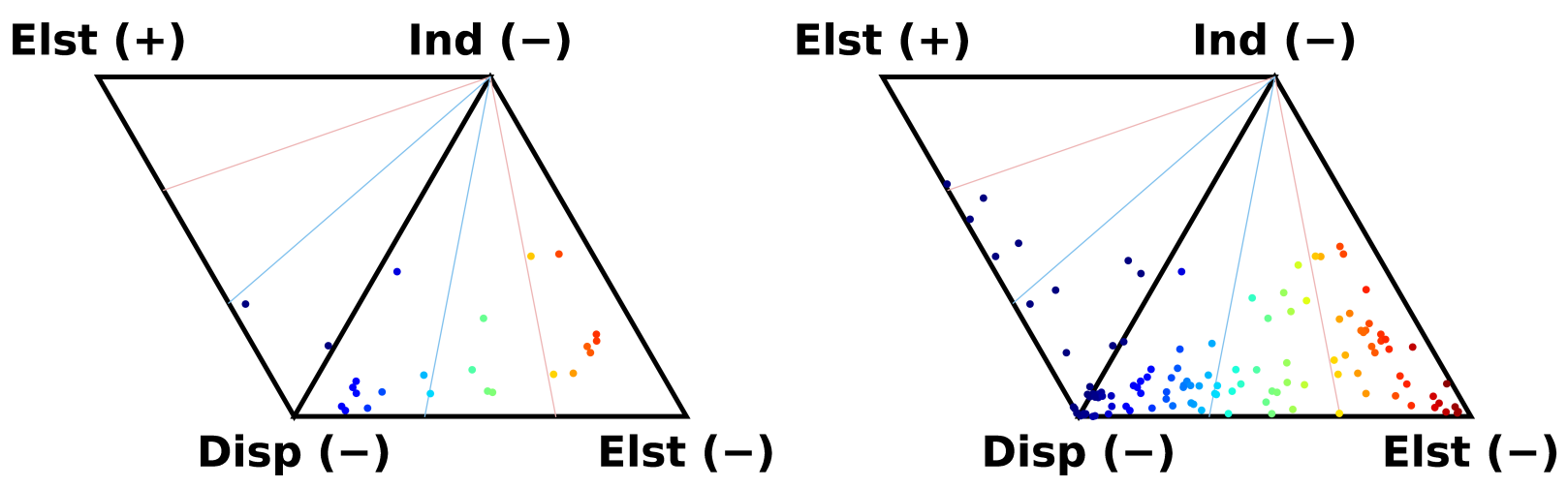

FIG. 1. Ternary diagrams of the $\mathrm{O} 24$ data set at $R=1.0 R_{\text {eq }}$ (left) and of the full $\mathrm{O} 24 \mathrm{x} 5$ data set (right). Diagrams color-coded by system type.

aug-cc-pV5Z basis sets ${ }^{79}$ were used. No extrapolation was applied to the Hartree-Fock (HF) energy component for which we used the aug-cc-pV5Z values. All reported DFT calculations were performed using the the aug-cc-pVQZ basis (doubly augmented in the case of $\mathrm{He}^{*}-\mathrm{He}^{*}$ ) with no CBS extrapolation.

Explicitly correlated CC calculations employed the aug-cc-pVTZ orbital basis and augpVTZ-JK ${ }^{80}$ and aug-cc-pVTZ-RI ${ }^{81}$ auxiliary basis sets for the self-consistent field and correlated components of the computations, respectively. To converge the interaction energy curve of $\mathrm{He}^{*}-\mathrm{He}^{*}$ we used the d-aug-cc-pVQZ orbital basis set accompanied by aug-ccpV6Z ${ }^{82}$ auxiliary basis sets.

All supermolecular interaction energies were corrected for the basis set superposition error (BSSE) with the counterpoise correction of Boys and Bernadi. ${ }^{83}$

As the error measure we used the capped unsigned relative error (CURE) and its mean value (MCURE), both introduced in Ref. 22

$$
\begin{aligned}
\operatorname{CURE}_{i} & =\frac{\left|E_{\text {int }, i}-E_{\text {int }, i}^{\mathrm{ref}}\right|}{E_{\text {weight }, i}} \cdot 100 \%, \\
E_{\text {weight }, i} & =\max \left\{\left|E_{\text {int }, i}^{\mathrm{ref}}\right|, \xi\left|E_{\text {int }, i}^{\mathrm{ref}, \mathrm{eq}}\right|\left(\frac{R_{i}^{\mathrm{eq} 3}}{R_{i}}\right)\right\} \\
\mathrm{MCURE} & =\frac{1}{N} \sum_{i}^{N} \mathrm{CURE}_{i}
\end{aligned}
$$

with $\xi=0.2$. This error measure avoids the problems related to the interaction energy curves crossing zero. ${ }^{13}$ To discuss the role of dispersion corrections in density functional 
approximations, we also report signed relative errors defined as

$$
\mathrm{SRE}_{i}=\frac{E_{\mathrm{int}, i}-E_{\mathrm{int}, i}^{\mathrm{ref}}}{E_{\mathrm{int}, i}^{\mathrm{ref}}} \cdot 100 \%,
$$

\section{RESULTS}

\section{A. Choice of the benchmark method}

For closed-shell systems the $\operatorname{CCSD}(\mathrm{T})$ method is widely accepted as the golden standard of quantum chemistry which reproduces noncovalent interactions. To achieve a significant improvement of the interaction energy over $\operatorname{CCSD}(\mathrm{T})$ one has to include full triples and contribution from noniterative quadrupoles, i.e., the CCSDT(Q) level of theory, dubbed recently by Kodrycka and Patkowski as the "platinum standard" of intermolecular interaction energy calculations. ${ }^{84}$ The cost of post-CCSD(T) corrections is too high for calculations bigger than 10-20 electrons, unless small basis sets are used. ${ }^{85}$ Small basis sets, however, are not recommended - as demonstrated by Smith et al. ${ }^{22}$ post-CCSD(T) contributions cannot be correctly recovered without adequate diffusion coefficients. Taking into account the prohibitive cost of post-CCSD $(\mathrm{T})$ approaches in adequate basis sets, we choose the $\operatorname{CCSD}(\mathrm{T})$ method as the reference for systems of the $\mathrm{O} 24 \mathrm{x} 5$ dataset. This level of accuracy is sufficient to provide a well-balanced and comprehensive reference for testing inexpensive quantum chemistry methods.

There are several formulations of the coupled-cluster method for high-spin open-shell systems, which differ in the expectation value of the $\hat{S}^{2}$ operator. In this work we use the spin-restricted coupled-cluster variants introduced by Knowles and co-authors, ${ }^{29,86,87}$ as implemented in the Molpro package. In the RCCSD/RCCSD $(\mathrm{T})$ method, also known as the partially spin-adapted (PSA) approach, only the linear term $(1+T) \Phi$ of the cluster expansion is spin-adapted ( $\Phi$ denotes the Hartree-Fock wave function). Additionally, we present results of ROHF-CCSD/CCSD $(T)^{62}$ calculations. Since the latter method is essentially not spin-adapted and the coupled-cluster wave function is spin-contaminated, we use the UCCSD/UCCSD(T) abbreviation, as is common in the literature. Rigorous spinadaptation schemes are also possible, ${ }^{88-90}$ but have not been explored here.

The spin adaptation procedures for $\operatorname{RCCSD}(\mathrm{T})$ method suffer from a minor violation of size-consistency for dimers which dissociate to two open-shell monomers. ${ }^{48,89}$ In our dataset 
such a behavior is observed for $\mathrm{NH}-\mathrm{NH}, \mathrm{Li}-\mathrm{O}_{2}$, and $\mathrm{O}_{2}-\mathrm{O}_{2}$ dimers. To account for sizeinconsistency, we apply uniform shift with respect to infinite separation of the monomers (calculations were performed for the intermonomer separation of $100 \AA$ ). The RCCSD values of the shifts in the aug-cc-pV5Z basis set are: $-1.13 \mathrm{~cm}^{-1}$ for $\mathrm{NH}-\mathrm{NH},-0.66 \mathrm{~cm}^{-1}$ for $\mathrm{Li}-\mathrm{O}_{2}$, and $-2.12 \mathrm{~cm}^{-1}$ for $\mathrm{O}_{2}-\mathrm{O}_{2}$. The corresponding $\mathrm{RCCSD}(\mathrm{T})$ shifts amount to

$0.15 \mathrm{~cm}^{-1}, 0.42 \mathrm{~cm}^{-1}$ and $1.06 \mathrm{~cm}^{-1}$. In Table S1 in the Supplementary Information we gathered the values of the interaction energy shifts for the F12 methods.

The UCCSD approach is size-consistent, but suffers from spin-contamination. Stanton ${ }^{91}$ found that this effect is small and the coupled cluster expectation values of the $\hat{S}^{2}$ operator are nearly exact. This is true even for molecules such as CN, which exhibit strong spin contamination of the UHF wave function. ${ }^{29,91-93}$ We verified that the UCCSD/UCCSD(T) spin contamination is below 0.01 for all systems of the $\mathrm{O} 24 \times 5$ dataset (see Table S2 in the supporting materials). (The UHF spin contaminaton is large for the $\mathrm{CN}$ and vinyl radicals.)

Among the discussed open-shell coupled-cluster formulations, we select the PSA RCCSD(T) method as benchmark. This approach is by far the most popular choice for accurate potential energy surface calculations. To recapitulate, PSA RCCSD $(T)$ avoids spin contamination, but may suffer from size-inconsistency when applied to interaction between two open-shell monomers. In this work size-inconsistency is addressed by a uniform shift in energy with respect to the dissociation limit. The reference $\mathrm{RCCSD}(\mathrm{T}) / \mathrm{CBS}$ data for the $\mathrm{O} 24 \mathrm{x} 5$ dataset are presented in Table IV. Based on a comparison with the $\operatorname{RCCSD}(\mathrm{T}) / \mathrm{CBS}+$ midbond results at $R / R_{\text {eq }}=1.0$, we estimate the basis-set error in the benchmark on the order of $2 \%$ (Figure S1 in the Supplementary Information).

\section{B. Wave function methods}

All correlated wave function methods are quantitatively correct for the interaction energy curves of the electrostatic subset of the O24x5 database. In Figure 2 we present MCUREs of orbital calculations (MP2, UMP2, RCCSD) and explicitly-correlated coupled-cluster calculations [RCCSD-F12a and RCCSD(T**)-F12a]. (See the Supplementary Information for all interaction energies.) The performance of MP2 and RCCSD are comparable, with errors below $5 \%$ for the equilibrium distance as well as larger separation between the monomers. The largest deviations from benchmark values occur at $R / R_{\mathrm{eq}}=0.9$ where MCUREs reach 


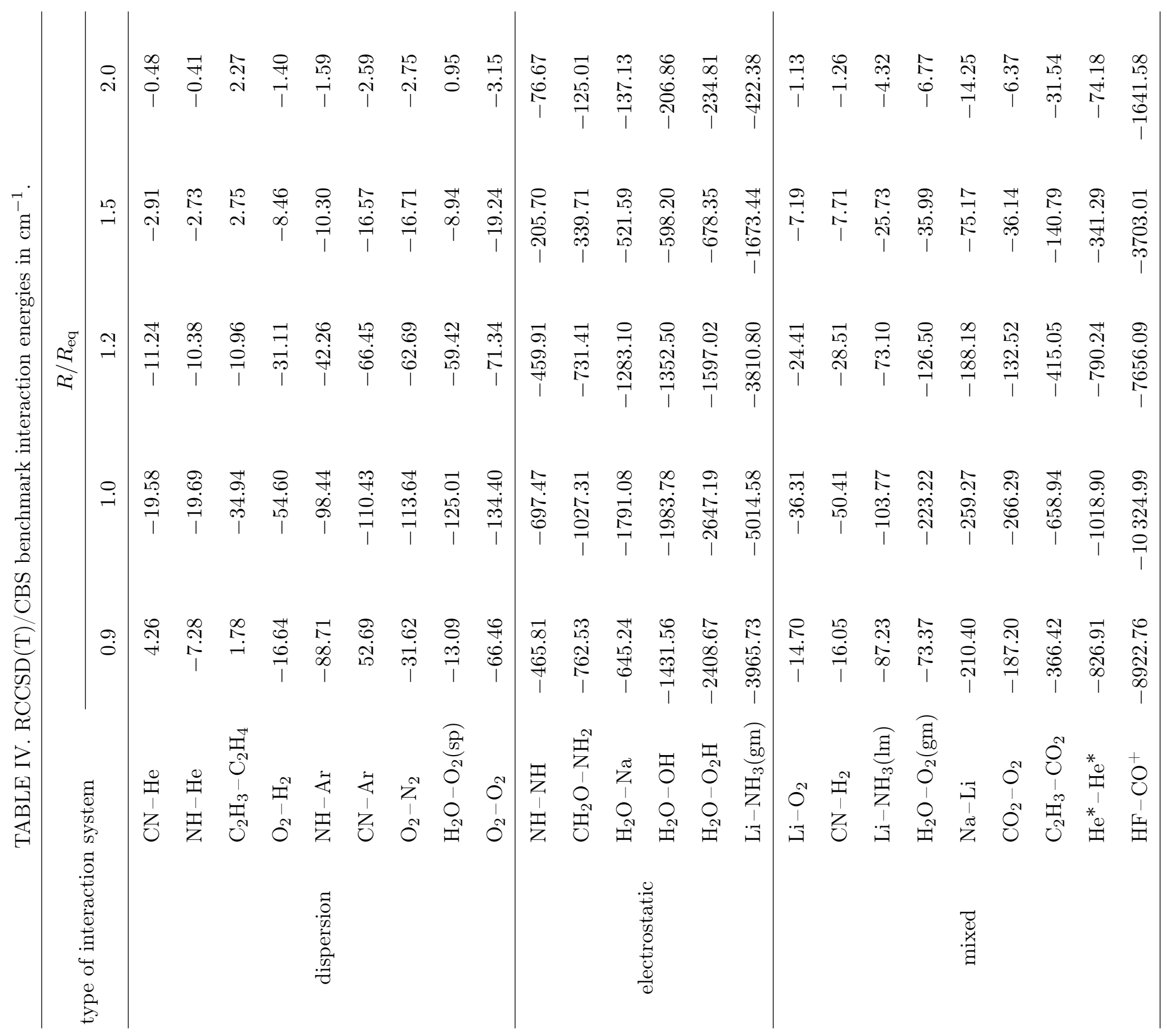



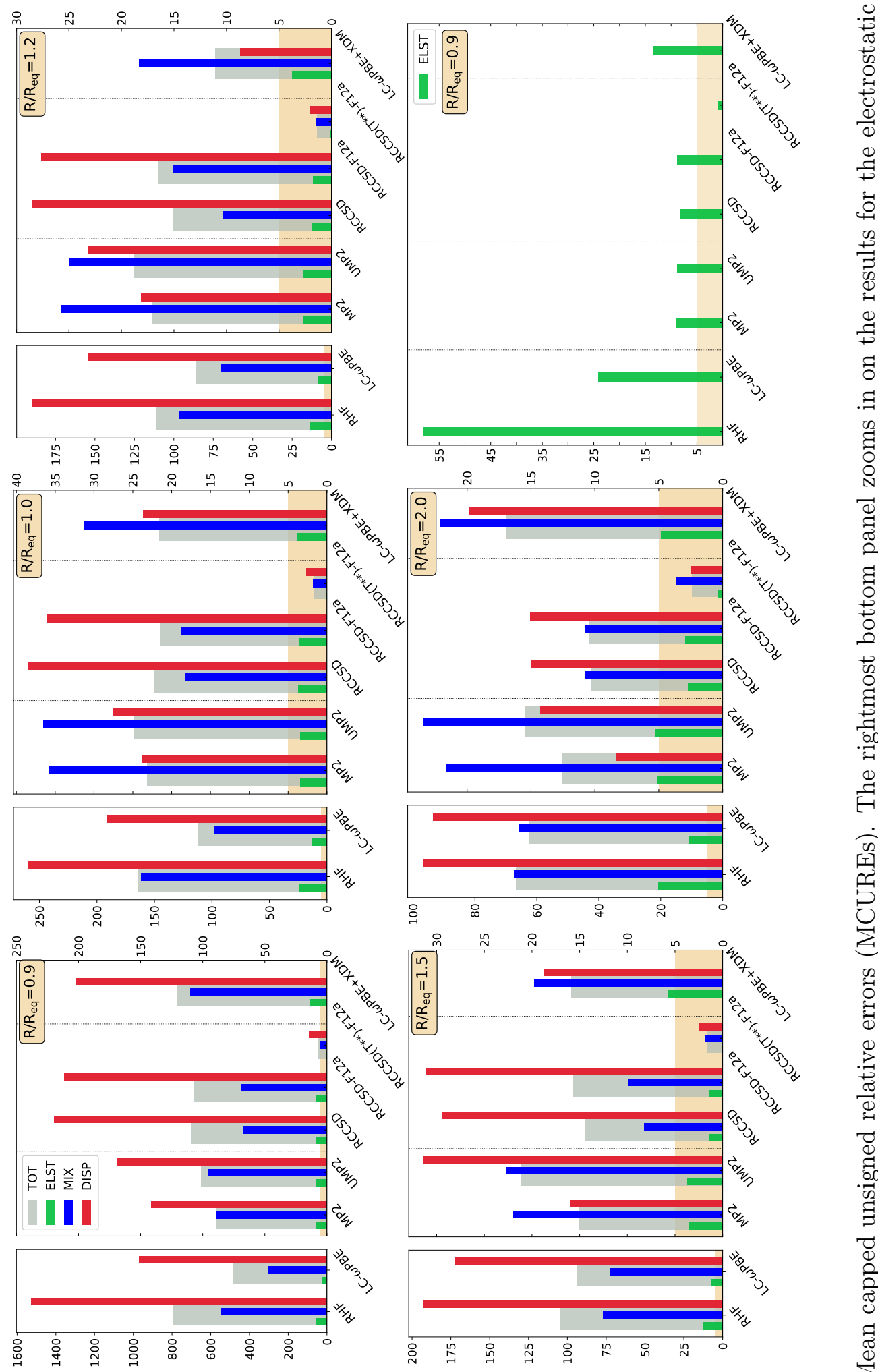

[\%] ヨyกวw
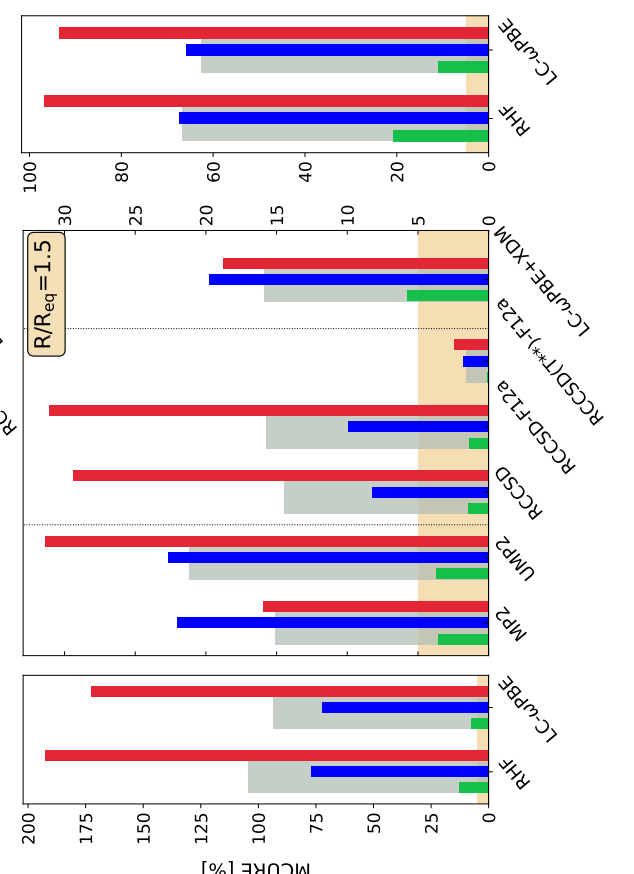

[\%] ヨyกวW

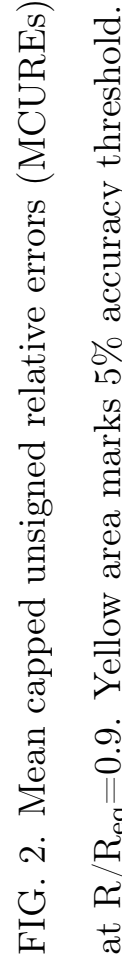


9.0\% and $8.3 \%$ for MP2 and RCCSD, respectively (see also Table S4 in the supporting materials).

The mixed and dispersion-dominated systems are much more difficult cases as they clearly require the inclusion of connected triples. The errors of MP2, UMP2 and RCCSD methods fall within the $20-36 \%$ range for $R / R_{\text {eq }}>0.9$. At $R / R_{\text {eq }}=0.9$ distance the accuracy is even worse with errors exceeding $200 \%$.

For several systems we observe large discrepancy between MP2 and UMP2 interaction energies. These dimers include $\mathrm{HF}-\mathrm{CO}^{+}, \mathrm{CN}-\mathrm{H}_{2}, \mathrm{CN}-\mathrm{He}, \mathrm{CN}-\mathrm{Ar}$, both $\mathrm{O}_{2}-\mathrm{H}_{2} \mathrm{O}$ complexes, $\mathrm{O}_{2}-\mathrm{O}_{2}, \mathrm{O}_{2}-\mathrm{H}_{2}$, and $\mathrm{O}_{2}-\mathrm{N}_{2}$. The largest deviation from the expected $S^{2}$ value occurs for $\mathrm{C}_{2} \mathrm{H}_{3}-\mathrm{C}_{2} \mathrm{H}_{4}\left(S_{\text {obs }}^{2}-S_{\text {exact }}^{2}=0.25\right.$ for both the dimer and the $\mathrm{C}_{2} \mathrm{H}_{3}$ molecule). In this case the difference between MP2 and UMP2 at $R / R_{\mathrm{eq}}=1$ is particularly pronounced $[\mathrm{MCURE}(\mathrm{MP} 2)=77 \%$ vs. MCURE $(\mathrm{UMP} 2)=166 \%]$. In all of the mentioned complexes the discrepancy between MP2 and UMP2 increases at shorter intermonomer separations. It should be noted that MP2 and UMP2 results remain in good agreement in the NH-containing dimers $(\mathrm{NH}-\mathrm{NH}, \mathrm{NH}-\mathrm{He}$, and $\mathrm{NH}-\mathrm{Ar})$ in spite of sizeable spin contamination diagnostics for the NH molecule.

To estimate the discrepancy between $\operatorname{UCCSD}(\mathrm{T})$ and $\operatorname{RCCSD}(\mathrm{T})$ we used the aug-ccpVTZ basis set supplemented with midbonds (see Table S15 in the Supplementary Information). The largest difference between these methods occurs at $R / R_{\mathrm{eq}}=0.9$ and 2.0 for $\mathrm{O}_{2}-\mathrm{H}_{2}, \mathrm{O}_{2}-\mathrm{N}_{2}, \mathrm{C}_{2} \mathrm{H}_{3}-\mathrm{C}_{2} \mathrm{H}_{4}, \mathrm{CN}-\mathrm{H}_{2}, \mathrm{CN}-\mathrm{Ar}, \mathrm{CN}-\mathrm{He}$, and $\mathrm{NH}-\mathrm{He}$. At short separations, $\mathrm{UCCSD}(\mathrm{T})$ deviates from RCCSD $(\mathrm{T})$ by as much as $20 \%\left(\mathrm{H}_{2} \mathrm{O}-\mathrm{O}_{2}\right.$ in global minimum). At long-range, the largest difference reaches $4 \%\left(\mathrm{CN}-\mathrm{H}_{2}\right)$. For the intermediate separations the differences between unrestricted and restricted approaches are on the order of $1 \%$ or lower (see Table S16 in the Supplementary Information).

Two dimers composed of highly polarizable monomers, i.e., $\mathrm{Na}-\mathrm{Li}$ and $\mathrm{He}^{*}-\mathrm{He}^{*}$, are outliers at the MP2 level of theory (see Tables S5-S9 in the supplementary materials). Their interaction energies are severely underestimated which we attribute to supermolecular MP2 recovering the dispersion interaction only at the level of noninteracting response functions ${ }^{94}$ (uncoupled response). For metals and He in the ${ }^{3} \mathrm{~S}$ state the uncoupled dispersion results in an underestimation of the dispersion energy, ${ }^{95}$ which is in contrast with the well-known MP2 overestimation of $\pi-\pi$ stacking interactions in closed-shell systems. ${ }^{96}$ After the uncoupled component is replaced with its coupled counterpart, ${ }^{97,98}$ the errors at $R / R_{\text {eq }}=2.0$ decrease 
from $64 \%$ and $72 \%$ to $3 \%$ and $2 \%$ for $\mathrm{Na}-\mathrm{Li}$ and $\mathrm{He}^{*}-\mathrm{He}^{*}$, respectively (see also Table S17 in the Supplementary Information).

The $\mathrm{H}_{2} \mathrm{O}-\mathrm{O}_{2}$ complex poses a particular challenge for both CCSD and MP2 approaches at $R / R_{\text {eq }}=1.5$ and 2.0 intermonomer separation. While this system is assigned to the dispersion subset according to the SAPT energy decomposition in the equilibrium, asymptotically it is dominated by the electrostatic interaction. The $\mathrm{H}_{2} \mathrm{O}-\mathrm{O}_{2}$ dimer is a transition state where the attractive dispersion and repulsive electrostatic components cancel each other and the interaction energy changes sign between $R / R_{\text {eq }}=1.5$ and $R / R_{\text {eq }}=2.0$. The interplay between dispersion and electrostatics is a subtle correlation effect. The beyond Hartree-Fock contribution to the dipole-quadrupole interaction at the CISD level of theory amounts to $-0.4 \mathrm{~cm}^{-1}$ which is roughly half of the interaction energy $\left(0.95 \mathrm{~cm}^{-1}\right)$. Similar cancellation takes place in the $\mathrm{O}_{2}-\mathrm{O}_{2}$ complex, but to a lesser degree due to the small magnitude of the quadrupole-quadrupole interaction.

The poor performance of MP2 for closed-shell dispersion-dominated complexes may be improved by scaling of the correlation energy spin components. ${ }^{99-101}$ We find that this does not work for open-shell system of the O24x5 database. The SCS-MP2 scheme applied with the original scaling factors $\left(c_{s s}=1 / 3, c_{o s}=6 / 5\right)$ yields ca. 2 times larger MCUREs for all types of interactions compared to MP2 (see Tables S5-S9 in the supplementary materials).

The CCSD(T)-F12 methods applied with triple-zeta basis sets are a frequent choice for noncovalent interactions computations. ${ }^{13,64,84,102,103}$ The common expectation is that with explicit correlation one gains at least two additional cardinal numbers in terms of the basis set convergence. ${ }^{104}$

Among the subsets of the $\mathrm{O} 24 \mathrm{x} 5$ database, $\operatorname{RCCSD}\left(\mathrm{T}^{* *}\right)-\mathrm{F} 12 \mathrm{a}$ and $\mathrm{RCCSD}\left(\mathrm{T}^{* *}\right)-\mathrm{F} 12 \mathrm{~b}$ perform best for the electrostatic subset where the errors stay below 1\% (Table V). For the mixed and dispersion subsets the errors are one order of magnitude larger. We observe that the more approximate $\operatorname{RCCSD}\left(\mathrm{T}^{* *}\right)-\mathrm{F} 12 \mathrm{a}$ variant applied with the aug-cc-pVTZ basis set outperforms RCCSD( $\left.\mathrm{T}^{* *}\right)$-F12b for all types of interactions. The unrestricted F12 variants give consistently worse results, especially for mixed and dispersion-dominated systems. Complexes for which a sizeable spin contamination coincides with a large deviation between restricted and unrestricted $\operatorname{CCSD}\left(\mathrm{T}^{* *}\right)-\mathrm{F} 12$ (between $15 \%$ and $27 \%$ in the minimum, respectively) comprise $\mathrm{NH}-\mathrm{He}, \mathrm{O}_{2}-\mathrm{H}_{2}, \mathrm{CN}-\mathrm{H}_{2}$ and $\mathrm{CN}-\mathrm{He}$. Similar to the MP2 case, the discrepancy is more pronounced as the monomers approach each other. The notable dif- 
ference with respect to UMP2 results is the $\mathrm{C}_{2} \mathrm{H}_{3}-\mathrm{C}_{2} \mathrm{H}_{4}$ dimer for which the discrepancy between $\operatorname{RCCSD}\left(\mathrm{T}^{* *}\right)$-F12 and $\operatorname{UCCSD}\left(\mathrm{T}^{* *}\right)$-F12 is neglibible.

\section{Density functional methods}

It has been recognized in the literature that the two major issues in the DFT description of noncovalent systems are its approximate treatment of the exchange contribution ${ }^{105}$ and the lack of an adequate account of long-range correlation. ${ }^{106}$ While those problems have been reported for closed-shell systems composed of main-group elements, they affect all types of noncovalent systems.

The DFT exchange artifacts manifest themselves in van der Waals complexes as an artificial binding by a semilocal exchange. ${ }^{105}$ In this work, we probe the dependence on the exchange energy by testing a series of exchange functionals while keeping them paired with the same PBE correlation. ${ }^{65}$ The exchange functionals we test differ in the way exact exchange is incorporated. They belong to three categories: pure functionals (PBE) ${ }^{65}$ global hybrids (PBE0) ${ }^{66}$ and long-range corrected hybrids (LC- $\left.\omega \mathrm{PBE}\right) \cdot{ }^{69,107}$ Although we limit ourselves to PBE, we believe that the conclusions inferred from our data hold for other semilocal models.

DFT dispersion corrections are additional terms added to hybrid and semilocal DFT to account for the long-range dispersion energy. A vast body of numerical evidence demonstrates that dispersion-corrected DFT, DFT + D for short, is useful and reliable for closedshell systems composed of main-group elements. ${ }^{108}$ Yet, little attention has been paid to the DFT description of the dispersion interaction in open shell systems. A notable exception is the work of Thonhauser et al. ${ }^{109}$ where the authors consider dispersion energy contributions in concert with spin effects in the adsorption of $\mathrm{CO}_{2}$ and $\mathrm{H}_{2}$ in metal-organic frameworks. The method of Ref. 109 is based on a spin-dependent formulation ${ }^{109}$ of the nonlocal van

der Waals density functional (vdW-DF). ${ }^{110,111}$ In our study, we consider another nonlocal density functional which is popular in the chemical community: the Vydrov-Van Voorhis functional, abbreviated as VV10. ${ }^{112}$

Another model of dispersion we consider is the exchange hole dipole moment (XDM) method of Becke and Johnson. ${ }^{113,114}$ One should mention that the XDM model has been successfully applied to construct a potential energy surface for the challenging open-shell 
TABLE V. Mean capped unsigned relative errors (MCUREs, in percent) for systems of the O24x5 basis set obtained with restricted open-shell and unrestricted explicitly correlated CC approaches in the F12a and F12b variants.

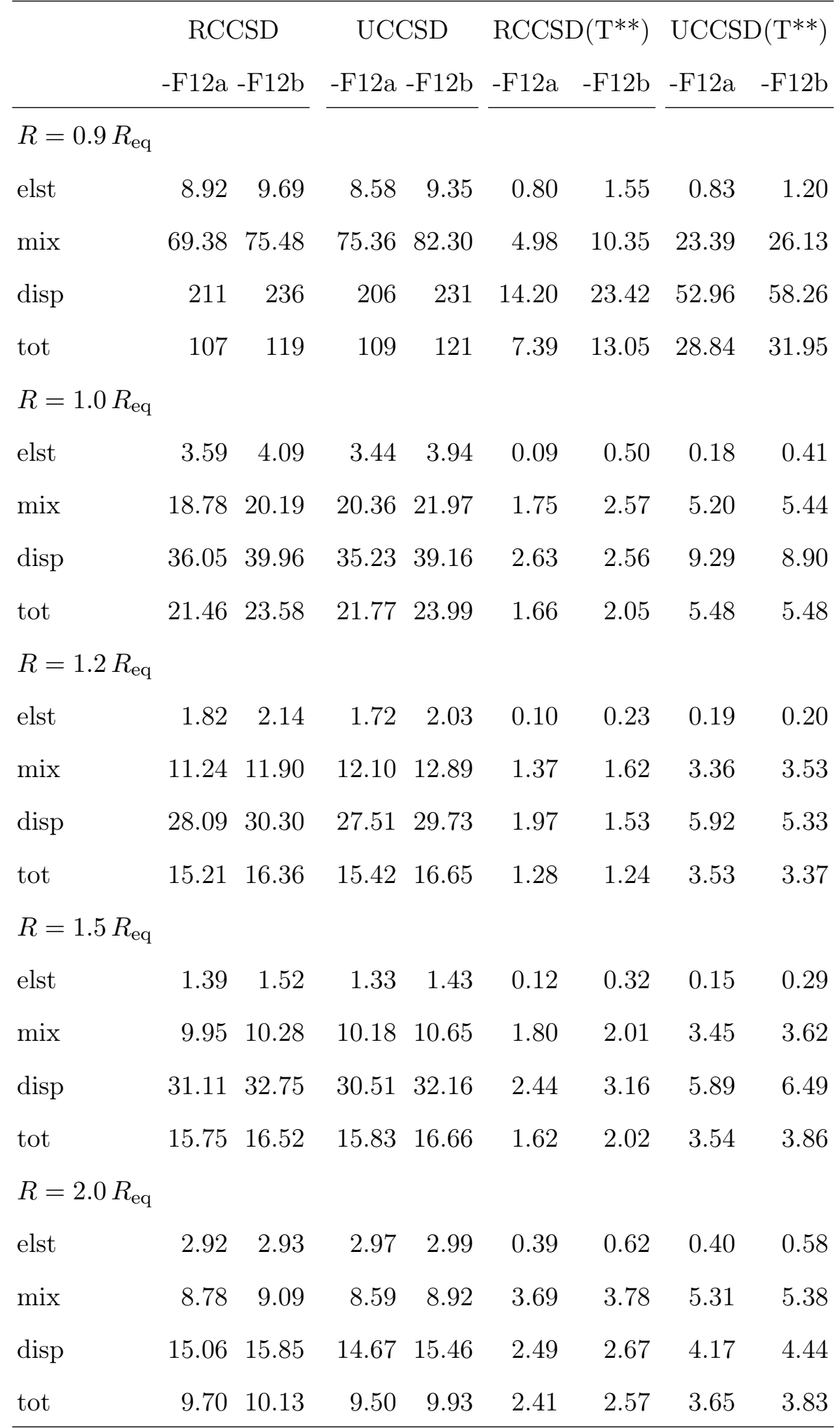


$\operatorname{Ar-NO}\left(X^{2} \Pi\right)$ dimer ${ }^{115}$ using a post-Hartree-Fock scheme of Becke and Johnson. ${ }^{116}$

Finally, DFT-D3 is a method which does not depend on the electron density explicitly but specifies the system via atomic positions and effective coordination numbers of atoms in a molecule. ${ }^{70}$ The $C_{6}$ coefficients on which D3 is based have been precomputed for both closed shell and open shell hydrides across the periodic table. ${ }^{70}$ Nonetheless, the functionaldependent parameters of the damping function have been optimized for closed-shell training sets of dimers, e.g., the S22 dataset. ${ }^{16,70}$ Although D3 is now the most popular choice of a $\mathrm{DFT}+\mathrm{D}$ method, to date it has not been systematically tested against open-shell reference data.

The problem of artificial exchange binding by DFT on the O24x5 dataset is severe. Whether the DFT interaction energies are attractive or repulsive strongly depends on the exchange functional. One expects that a functional not corrected for dispersion underbinds which would make it compatible with a subsequent addition of an attractive dispersion term. However, pure PBE energy without any dispersion correction overestimates the interaction energy of nine systems at their equilibrium bond lengths. In turn, the dispersion-corrected PBE method overestimates almost all interaction energies on the dataset (Figure 3). The addition of a fraction of orbital exchange in PBE0 reduces the attractive character but is far from eliminating it completely. The overbinding persists upon addition of any dispersion correction to PBE0; the only examples of slightly underbound equilibrium dimers are $\mathrm{O}_{2}-\mathrm{O}_{2}$ and $\mathrm{H}_{2} \mathrm{O}-\mathrm{O}_{2}(\mathrm{~min})$. The long-range corrected exchange, LC- $\omega \mathrm{PBE}$, is strongly repulsive and, therefore, compatible with dispersion corrections.

We identify the LC- $\omega \mathrm{PBE}+\mathrm{XDM}$ functional as the best dispersion-corrected variant for the equilibrium dimers of the $\mathrm{O} 24 \times 5$ database. The corresponding mean absolute error, $\bar{\Delta}_{\text {abs }}$, amounts to $21 \%$ and the standard deviation, $\sigma$, is $23 \%$ (see also Figure 3 for a box plot representation of errors). The performance of the remaining dispersion-corrected functionals based on LC- $\omega$ PBE is slightly worse: LC-VV10 $\left(\bar{\Delta}_{\text {abs }}=29 \%, \sigma=26 \%\right)$ and LC- $\omega$ PBE-D3 $\left(\bar{\Delta}_{\text {abs }}=29 \%, \sigma=42 \%\right)$. At long distances, $1.5 R_{\text {eq }}$ and $2.0 R_{\text {eq }}$, the accuracy is largely determined by the quality of the $C_{6}$ coefficients in a particular model of dispersion. As evident from Figure 3, D3 performs better than both XDM and VV10 at large separations. The superiority of D3 over the other methods in the long-range region is consistent with what has been reported by Shahbaz and Szalewicz for closed shell dimers. ${ }^{117}$

In Figure 4 we present the percent errors of $\mathrm{LC}-\omega \mathrm{PBE}+\mathrm{XDM}$ resolved into a series 

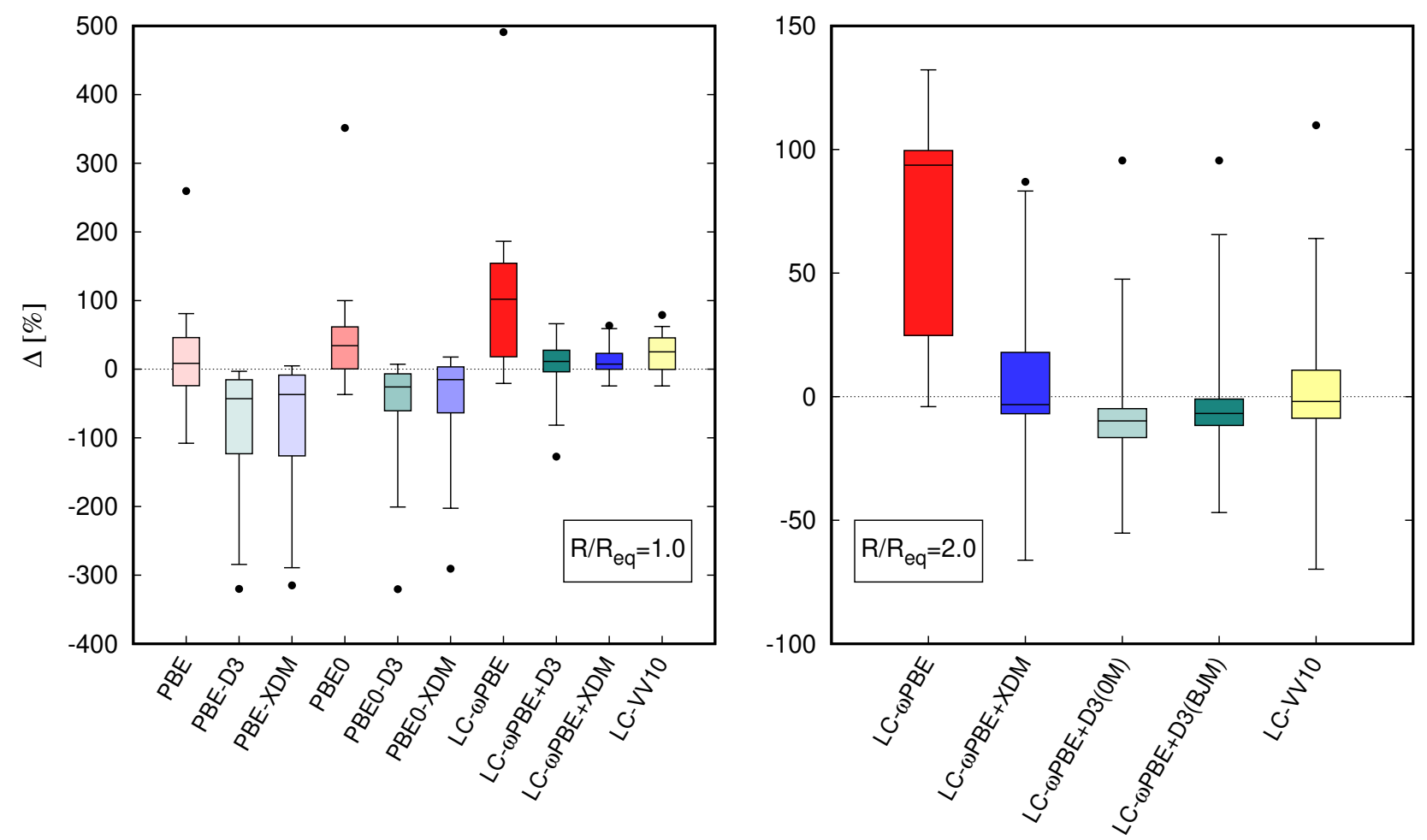

FIG. 3. Box plots of signed relative percent errors (SREs) in the calculated interaction energies for dimers of the $\mathrm{O} 24 \times 5$ dataset in the equilibrium geometry $\left(R=R_{\mathrm{eq}}\right.$, left) and at $R=2 R_{\mathrm{eq}}$ geometry (right). The D3(0M) and D3(BJM) labels denote the revised D3 correction ${ }^{22}$ applied either with zero- or Becke-Johnson damping, respectively. Errors are given with respect to the RCCSD(T)/CBS reference. The box and outer fences encompass $50 \%$ and $95 \%$ of the distribution, respectively.

of distances. At the shortest distance, $0.9 R_{\text {eq }}$, the deviation from the RCCSD(T)/CBS benchmark is on the order of $100 \%$ for the mixed and dispersion-dominated subsets, which is unacceptable. While it is expected that the accuracy deteriorates at short range, the error here is several times greater than the typical errors for closed shells. For instance, Smith et al. have reported the average errors of $12 \%$ at short distances for LC- $\omega$ PBE-D3 on an extensive database of closed-shell dimers. ${ }^{22}$ At equilibrium separations, DFT is still inaccurate. Observed errors at short and equilibrium distances suggests their possible origin in the exchange energy and the damping function of the dispersion correction. As noted by Shahbaz and Szalewicz, ${ }^{117,118}$ DFT dispersion corrections describe not only the physical contributions but also cancel out the approximate exchange artifacts. It is plausible that this cancellation is less effective for open-shell densities, e.g., because of an insufficient 

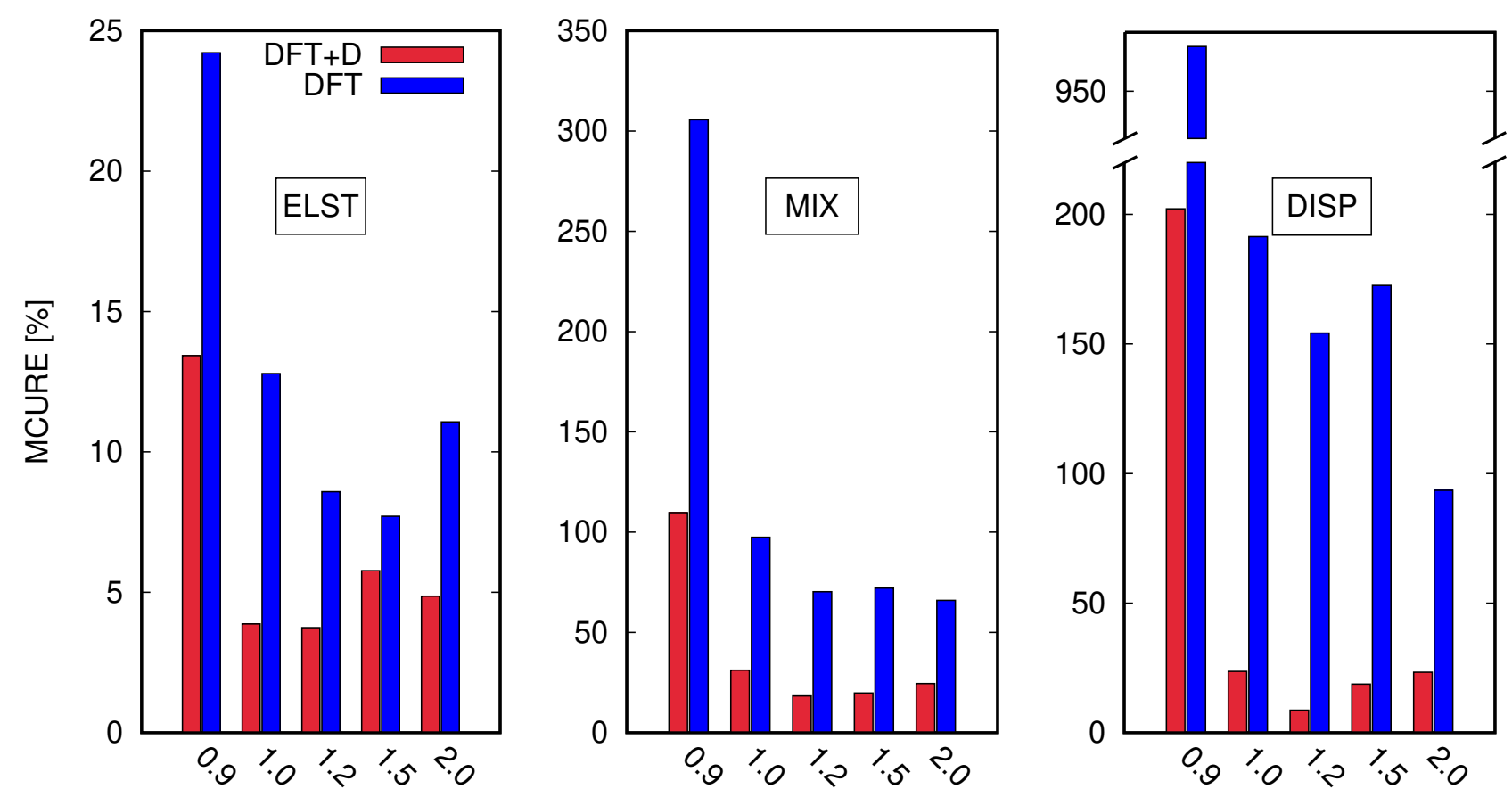

FIG. 4. Mean capped unsigned relative errors (MCUREs, in percent). DFT and DFT $+\mathrm{D}$ denote LC- $\omega \mathrm{PBE}$ and LC- $\omega \mathrm{PBE}+\mathrm{XDM}$ results, respectively.

parametrization on open-shell data.

We note in passing that it is impossible for a force field-like DFT dispersion correction to account for the exchange-dispersion contribution which couples the open-shell monomers to a specific spin state of a dimer. The importantce of that and the remaining limitations remains to be investigated in detail.

\section{CONCLUSIONS}

We introduced an interaction energy dataset of 24 open-shell noncovalent dimers. The systems were chosen to assure a good coverage of the ternary diagram, to guarantee diversity with respect to types of interactions: dispersion, electrostatics and induction. Each complex is represented by five data points obtained by a rigid translation of the molecules $\left(R / R_{\text {eq }} \in\right.$ $\{0.9,1.0,1.2,1.5,2.0\})$. We limited ourselves to small, high-spin dimers (up to eleven atoms) for which we provide the RCCSD(T)/CBS benchmark. The O24x5 database probes a wide range of interaction energies: from $-700 \mathrm{~cm}^{-1}$ to $-4900 \mathrm{~cm}^{-1}$ for the electrostatic subset, from $-35 \mathrm{~cm}^{-1}$ to $-100 \mathrm{~cm}^{-1}$ for the mixed subset (without $\mathrm{HF} \cdots \mathrm{CO}^{+}$), and from $-21 \mathrm{~cm}^{-1}$ 
to $-140 \mathrm{~cm}^{-1}$ for the dispersion dominated systems.

We assessed the accuracy of several approximate wave function methods (MP2, UMP2, RCCSD, UCCSD, UCCSD(T), RCCSD(T**)-F12 and UCCSD(T**)-F12 in F12a and F12b variants) and density functionals based on the PBE exchange-correlation model. The dispersion-dominated and mixed systems proved to be the most challenging complexes. For $R / R_{\mathrm{eq}} \geq 1.0$ in these subsets the errors of both restricted and unrestricted MP2 and CCSD methods fall in the 20-40\% range. The accuracy of the best-performing PBE-based model, LC- $\omega \mathrm{PBE}+\mathrm{XDM}$, is similar to MP2. Explicitly correlated approaches which include contributions from connected triples in the $\left(\mathrm{T}^{* *}\right)$ approximation reproduce the $\mathrm{RCCSD}(\mathrm{T}) / \mathrm{CBS}$ benchmark with errors in the $2-5 \%$ range for both dispersion and mixed subsets in the augmented triple-zeta basis set. For $R / R_{\text {eq }}=0.9$ all approximate approaches yield errors roughly one order of magnitude larger compared to the remaining distances.

The errors on the electrostatic subset are typically 5 up to 10 times smaller than for mixed and dispersion-dominated systems. In this subset both MP2 and CCSD wave function methods as well as the LC- $\omega \mathrm{PBE}+\mathrm{XDM}$ dispersion-corrected functional are accurate to within $6 \%$ at $R / R_{\text {eq }} \geq 1.0$ configurations. The $\operatorname{RCCSD}\left(\mathrm{T}^{* *}\right)-\mathrm{F} 12$ approaches in a triple-zeta basis set afford accuracy better than $1 \%$. The relative errors of the explicitly correlated results at $R / R_{\text {eq }} \geq 1.0$ amount to $0.1 \%$ and $0.5 \%$ for the $\mathrm{F} 12 \mathrm{a}$ and $\mathrm{F} 12 \mathrm{~b}$ variants, respectively. Similar to the dispersion and mixed subsets, the accuracy of all tested wave function and DFT methods at $R / R_{\text {eq }}=0.9$ for the electrostatic subset deteriorates by roughly one order of magnitude compared to larger intermonomer separtions.

The $024 \times 5$ database features several complexes with considerable spin-contamination in unrestricted MP2 and CCSD methods: the CN-containing dimers ( $\mathrm{CN}-\mathrm{He}, \mathrm{CN}-\mathrm{Ar}$, $\left.\mathrm{CN}-\mathrm{H}_{2}\right), \mathrm{O}_{2}$-containing dimers $\left(\mathrm{O}_{2}-\mathrm{N}_{2}, \mathrm{O}_{2}-\mathrm{O}_{2}\right)$ and $\mathrm{C}_{2} \mathrm{H}_{3}-\mathrm{C}_{2} \mathrm{H}_{4}$. For these systems the unrestricted MP2 and CC approaches perform worse than their restricted counterparts. For example, the relative signed error of $\mathrm{RCCSD}\left(\mathrm{T}^{* *}\right)-\mathrm{F} 12 \mathrm{a}$ for equilibrium $\mathrm{CN}-\mathrm{H}_{2}$ is $-2 \%$, whereas for the unrestricted variant the error reaches $-30 \%$.

To summarize, the $\mathrm{O} 24 \times 5$ dataset features noncovalent open-shell interactions of various physical origin and spans a wide range of interaction strengths. In particular, the dataset contains challenging dispersion-dominated and mixed-type subsets for which any approximate method that does not include the $(\mathrm{T})$ contribution yields errors on the order of tens of percent. We expect that the $\mathrm{O} 24 \times 5$ dataset will become a valuable addition to benchmark- 
ing protocols used for wave function approaches and training sets for semiempirical methods and model density functionals.

\section{ACKNOWLEDGMENTS}

This research was supported in part by PLGrid Infrastructure. We are also grateful for the resources provided by the Wroclaw Centre for Networking and Supercomputing grant 218. M.H. would like to thank Dominika Zgid for her hospitality during the Kosciuszko Foundation scholarship. P.S.Z., L.R., K.M. and F.B. are grateful to National Science Center for support (Opus10, number 2015/19/B/ST4/02707). We also thank Grzegorz Chałasiński for reading and commenting on this manuscript.

\section{DATA AVAILABILITY}

The results of all calculations along with geometries (in xyz format) are available at the GITLAB repository https://gitlab.com/piotr.zuchowski/o24x5.

\section{REFERENCES}

${ }^{1}$ G. Chałasiński and M. M. Szczęśniak, "State of the Art and Challenges of the ab initio Theory of Intermolecular Interactions," Chem. Rev. 100, 4227-4252 (2000).

${ }^{2}$ E. Roueff and F. Lique, "Molecular Excitation in the Interstellar Medium: Recent Advances in Collisional, Radiative, and Chemical Processes," Chem. Rev. 113, 8906-8938 (2013).

${ }^{3}$ T. Karman, M. A. Koenis, A. Banerjee, D. H. Parker, I. E. Gordon, A. Avoird, W. J. Zande, and G. C. Groenenboom, " $\mathrm{O}_{2}-\mathrm{O}_{2}$ and $\mathrm{O}_{2}-\mathrm{N}_{2}$ collision-induced absorption mechanisms unravelled," Nat. Chem. 10, 549 (2018).

${ }^{4}$ J. Jankunas, K. Jachymski, M. Hapka, and A. Osterwalder, "Observation of orbiting resonances in $\mathrm{He}\left({ }^{3} \mathrm{~S}_{1}\right)+\mathrm{NH}_{3}$ Penning ionization." J. Chem. Phys. 142, 164305 (2015).

${ }^{5}$ E. Lavert-Ofir, Y. Shagam, A. B. Henson, S. Gersten, J. Kłos, P. S. Żuchowski, J. Narevicius, and E. Narevicius, "Observation of the isotope effect in sub-kelvin reactions." Nat. Chem. 6, 332-5 (2014). 
${ }^{6}$ M. Lara, J. L. Bohn, D. Potter, P. Soldán, and J. M. Hutson, "Ultracold Rb-OH Collisions and Prospects for Sympathetic Cooling," Phys. Rev. Lett. 97, 183201 (2006).

${ }^{7}$ P. S. Żuchowski and J. M. Hutson, "Prospects for producing ultracold $\mathrm{NH}_{3}$ molecules by sympathetic cooling: A survey of interaction potentials," Phys. Rev. A 78, 022701 (2008). ${ }^{8}$ M. Morita, M. B. Kosicki, P. S. Żuchowski, and T. V. Tscherbul, "Atom-molecule collisions, spin relaxation, and sympathetic cooling in an ultracold spin-polarized $\operatorname{Rb}\left({ }^{2} S\right)$ $\operatorname{SrF}\left({ }^{2} \Sigma^{+}\right)$mixture," Phys. Rev. A 98, 042702 (2018).

${ }^{9}$ S. N. Steinmann and C. Corminboeuf, "Exploring the Limits of Density Functional Approximations for Interaction Energies of Molecular Precursors to Organic Electronics," J. Chem. Theory Comput. 8, 4305-4316 (2012).

${ }^{10}$ I. Alcón, D. Reta, I. d. P. R. Moreira, and S. T. Bromley, "Design of multi-functional 2D open-shell organic networks with mechanically controllable properties," Chem. Sci. 8, 1027-1039 (2017).

${ }^{11}$ Y. Huang and E. Egap, "Open-shell organic semiconductors: an emerging class of materials with novel properties," Polym. J. , 1 (2018).

${ }^{12}$ J. Řezáč and P. Hobza, "Benchmark calculations of interaction energies in noncovalent complexes and their applications," Chem. Rev. 116, 5038-5071 (2016).

${ }^{13}$ K. Patkowski, "Chapter one - benchmark databases of intermolecular interaction energies: Design, construction, and significance," in Annu. Rep. Comput. Chem., Vol. 13, edited by D. A. Dixon (Elsevier, 2017) pp. $3-91$.

${ }^{14}$ C. D. Sherrill, T. Takatani, and E. G. Hohenstein, "An Assessment of Theoretical Methods for Nonbonded Interactions: Comparison to Complete Basis Set Limit CoupledCluster Potential Energy Curves for the Benzene Dimer, the Methane Dimer, BenzeneMethane, and Benzene-H${ }_{2}$ S," J. Phys. Chem. A 113, 10146-10159 (2009).

${ }^{15}$ M. S. Marshall, L. A. Burns, and C. D. Sherrill, "Basis set convergence of the coupledcluster correction, $\delta \mathrm{MP} 2 \mathrm{CCSD}(\mathrm{T})$ : Best practices for benchmarking non-covalent interactions and the attendant revision of the S22, NBC10, HBC6, and HSG databases," J. Chem. Phys. 135, 194102 (2011).

${ }^{16}$ P. Jurečka, J. Šponer, J. Černỳ, and P. Hobza, "Benchmark database of accurate (MP2 and $\operatorname{CCSD}(\mathrm{T})$ complete basis set limit) interaction energies of small model complexes, DNA base pairs, and amino acid pairs," Phys. Chem. Chem. Phys. 8, 1985-1993 (2006). 
${ }^{17}$ T. Takatani, E. G. Hohenstein, M. Malagoli, M. S. Marshall, and C. D. Sherrill, "Basis set consistent revision of the S22 test set of noncovalent interaction energies," J. Chem. Phys. 132, 144104 (2010).

${ }^{18}$ R. Podeszwa, K. Patkowski, and K. Szalewicz, "Improved interaction energy benchmarks for dimers of biological relevance," Phys. Chem. Chem. Phys. 12, 5974-5979 (2010).

${ }^{19}$ L. Gráfová, M. Pitonak, J. Rezac, and P. Hobza, "Comparative Study of Selected Wave Function and Density Functional Methods for Noncovalent Interaction Energy Calculations Using the Extended S22 Data Set," J. Chem. Theory Comput. 6, 2365-2376 (2010).

${ }^{20}$ D. E. Taylor, J. G. Ángyán, G. Galli, C. Zhang, F. Gygi, K. Hirao, J. W. Song, K. Rahul, O. Anatole von Lilienfeld, R. Podeszwa, I. W. Bulik, T. M. Henderson, G. E. Scuseria, J. Toulouse, R. Peverati, D. G. Truhlar, and K. Szalewicz, "Blind test of densityfunctional-based methods on intermolecular interaction energies," J. Chem. Phys. 145, 124105 (2016).

${ }^{21}$ J. Řezáč, K. E. Riley, and P. Hobza, "S66: A well-balanced database of benchmark interaction energies relevant to biomolecular structures," J. Chem. Theory Comput. 7, 2427-2438 (2011).

${ }^{22}$ D. G. A. Smith, L. A. Burns, K. Patkowski, and C. D. Sherrill, "Revised Damping Parameters for the D3 Dispersion Correction to Density Functional Theory," J. Phys. Chem. Lett. 7, 2197-2203 (2016).

${ }^{23} \mathrm{P}$. R. Tentscher and J. S. Arey, "Binding in radical-solvent binary complexes: Benchmark energies and performance of approximate methods," J. Chem. Theory Comput. 9, 15681579 (2013).

${ }^{24} \mathrm{P}$. R. Tentscher and J. S. Arey, "On the nature of interactions of radicals with polar molecules," J. Phys. Chem. A 117, 12560-12568 (2013).

${ }^{25}$ B. Alday, R. Johnson, J. Li, and H. Guo, "Hemibond complexes between $\mathrm{H}_{2} \mathrm{~S}$ and free radicals $(\mathrm{F}, \mathrm{Cl}, \mathrm{Br}$, and $\mathrm{OH})$," in Guosen Yan: A Festschrift from Theoretical Chemistry Accounts, edited by H. Guo, D. Xie, and W. Yang (Springer Berlin Heidelberg, Berlin, Heidelberg, 2015) pp. 177-182.

${ }^{26}$ E. Soydaş and U. Bozkaya, "Accurate Open-Shell Noncovalent Interaction Energies from the Orbital-Optimized Møller-Plesset Perturbation Theory: Achieving CCSD Quality at the MP2 Level by Orbital Optimization," J. Chem. Theory Comput. 9, 4679 (2013). 
${ }^{27}$ P. S. Żuchowski, R. Podeszwa, R. Moszyński, B. Jeziorski, and K. Szalewicz, "Symmetryadapted perturbation theory utilizing density functional description of monomers for highspin open-shell complexes," J. Chem. Phys. 129, 084101 (2008).

${ }^{28}$ M. Hapka, P. S. Żuchowski, M. M. Szczȩśniak, and G. Chałasiński, "Symmetry-adapted perturbation theory based on unrestricted Kohn-Sham orbitals for high-spin open-shell van der Waals complexes." J. Chem. Phys. 137, 164104 (2012).

${ }^{29}$ P. J. Knowles, C. Hampel, and H. J. Werner, "Coupled cluster theory for high spin, open shell reference wave functions," J. Chem. Phys. 99, 5219-5227 (1993).

${ }^{30}$ P. J. Knowles, C. Hampel, and H.-J. Werner, "Erratum: "coupled cluster theory for high spin, open shell reference wave functions" [ j. chem. phys. 99, 5219 (1993)]," J. Chem. Phys. 112, 3106-3107 (2000).

${ }^{31}$ I. Gordon, L. Rothman, C. Hill, R. Kochanov, Y. Tan, P. Bernath, M. Birk, V. Boudon, A. Campargue, K. Chance, B. Drouin, J.-M. Flaud, R. Gamache, J. Hodges, D. Jacquemart, V. Perevalov, A. Perrin, K. Shine, M.-A. Smith, J. Tennyson, G. Toon, H. Tran, V. Tyuterev, A. Barbe, A. Császár, V. Devi, T. Furtenbacher, J. Harrison, J.-M. Hartmann, A. Jolly, T. Johnson, T. Karman, I. Kleiner, A. Kyuberis, J. Loos, O. Lyulin, S. Massie, S. Mikhailenko, N. Moazzen-Ahmadi, H. Müller, O. Naumenko, A. Nikitin, O. Polyansky, M. Rey, M. Rotger, S. Sharpe, K. Sung, E. Starikova, S. Tashkun, J. V. Auwera, G. Wagner, J. Wilzewski, P. Wcisło, S. Yu, and E. Zak, "The HITRAN2016 molecular spectroscopic database," J. Quant. Spectrosc. Radiat. Transf. 203, 3 - 69 (2017), HITRAN2016 Special Issue.

${ }^{32}$ M.-L. Dubernet, M. Alexander, Y. Ba, N. Balakrishnan, C. Balança, C. Ceccarelli, J. Cernicharo, F. Daniel, F. Dayou, M. Doronin, et al., "BASECOL2012: A collisional database repository and web service within the Virtual Atomic and Molecular Data Centre (VAMDC)," Astron. Astrophys. 553, A50 (2013).

${ }^{33}$ V. Barone, C. Latouche, D. Skouteris, F. Vazart, N. Balucani, C. Ceccarelli, and B. Lefloch, "Gas-phase formation of the prebiotic molecule formamide: insights from new quantum computations," Mon. Not. R. Astron. Soc. 453, L31-L35 (2015).

${ }^{34}$ P. S. Żuchowski, "Interaction potential for the quintet state of the $\mathrm{O}_{2}-\mathrm{O}_{2}$ dimer from symmetry-adapted perturbation theory based on DFT description of monomers," Chem. Phys. Lett. 450, 203-209 (2008). 
${ }^{35}$ Y. Ikabata and H. Nakai, "Assessment of local response dispersion method for open-shell systems," Chem. Phys. Lett. 556, 386 - 392 (2013).

${ }^{36}$ N. J. Singh, S. K. Min, D. Y. Kim, and K. S. Kim, "Comprehensive energy analysis for various types of p-interaction," J. Chem. Theory Comput. 5, 515-529 (2009).

${ }^{37}$ L. A. Burns, M. S. Marshall, and C. D. Sherrill, "Appointing silver and bronze standards for noncovalent interactions: A comparison of spin-component-scaled (SCS), explicitly correlated (F12), and specialized wavefunction approaches," J. Chem. Phys. 141, 23411 (2014).

${ }^{38}$ J. F. Gonthier and C. D. Sherrill, "Density-fitted open-shell symmetry-adapted perturbation theory and application to $\pi$-stacking in benzene dimer cation and ionized DNA base pair steps," J. Chem. Phys. 145, 134106 (2016).

${ }^{39}$ F. Lique and J. Kłos, "Hyperfine excitation of CN by He," Monthly Notices of the Royal Astronomical Society: Letters 413, L20-L23 (2011).

${ }^{40}$ H. Cybulski, R. V. Krems, H. R. Sadeghpour, A. Dalgarno, J. Kłos, G. C. Groenenboom, A. van der Avoird, D. Zgid, and G. Chałasiński, "Interaction of $\mathrm{NH}\left(\mathrm{X}^{3} \Sigma^{-}\right)$with He: Potential energy surface, bound states, and collisional Zeeman relaxation," J. Chem. Phys. 122, 094307 (2005).

${ }^{41}$ R. Toboła, F. Dumouchel, J. Kłos, and F. Lique, "Calculations of fine-structure resolved collisional rate coefficients for the $\mathrm{NH}\left(\mathrm{X}^{3} \Sigma^{-}\right)$-He system," J. Chem. Phys. 134, 024305 (2011).

${ }^{42}$ Y. Kalugina, O. D. Alpizar, T. Stoecklin, and F. Lique, "A new ab initio potential energy surface for the collisional excitation of $\mathrm{O}_{2}$ by $\mathrm{H}_{2}$," Phys. Chem. Chem. Phys. 14, 16458-16466 (2012).

${ }^{43}$ R. A. Kendall, G. Chałasiński, J. Kłos, R. Bukowski, M. W. Severson, M. M. Szczęśniak, and S. M. Cybulski, "Ab initio study of the van der Waals interaction of $\mathrm{NH}\left(X^{3} \Sigma^{-}\right)$with $\operatorname{Ar}\left({ }^{1} S\right), "$ J. Chem. Phys. 108, 3235-3242 (1998).

${ }^{44}$ J. Han, M. C. Heaven, U. Schnupf, and M. H. Alexander, "Experimental and theoretical studies of the CN-Ar van der Waals complex," J. Chem. Phys. 128, 104308 (2008).

${ }^{45}$ M. Bartolomei, E. Carmona-Novillo, M. I. Hernández, J. Campos-Martínez, and R. Moszynski, "Global ab Initio Potential Energy Surface for the $\mathrm{O}_{2}\left({ }^{3} \Sigma_{g}^{-}\right)+\mathrm{N}_{2}\left({ }^{1} \Sigma_{g}^{+}\right)$ Interaction. Applications to the Collisional, Spectroscopic, and Thermodynamic Properties of the Complex," J. Phys. Chem. A 118, 6584-6594 (2014). 
${ }^{46}$ K. M. Dreux and G. S. Tschumper, "Anchoring the potential energy surface of an important atmospheric van der Waals dimer, the $\mathrm{H}_{2} \mathrm{O}-\mathrm{O}_{2}$ complex," Comput. Theor. Chem. 1072, $21-27$ (2015).

${ }^{47}$ M. Bartolomei, E. Carmona-Novillo, M. I. Hernández, J. Campos-Martínez, and R. Hernández-Lamoneda, "Global ab initio potential energy surfaces for the $\mathrm{O}_{2}\left({ }^{3} \Sigma_{g}^{-}\right)+\mathrm{O}_{2}\left({ }^{3} \Sigma_{g}^{-}\right)$interaction," J. Chem. Phys. 133, 124311 (2010).

${ }^{48}$ L. M. C. Janssen, G. C. Groenenboom, A. van der Avoird, P. S. Żuchowski, and R. Podeszwa, "Ab initio potential energy surfaces for $\mathrm{NH}\left({ }^{3} \Sigma^{-}\right)-\mathrm{NH}\left({ }^{3} \Sigma^{-}\right)$with analytical long range," J. Chem. Phys. 131, 224314 (2009).

${ }^{49}$ Hashimoto, Kenro and Kamimoto, Tetsuya and Daigoku, Kota, "Theoretical study of $\left[\mathrm{na}\left(\mathrm{h}_{2} \mathrm{O}\right)_{n}\right]^{-}(\mathrm{n}=1-4)$ clusters: Geometries, vertical detachment energies, and ir spectra,"

${ }^{50}$ S. Du, J. S. Francisco, G. K. Schenter, T. D. Iordanov, B. C. Garrett, M. Dupuis, and J. Li, "The $\mathrm{OH}$ radical- $\mathrm{H}_{2} \mathrm{O}$ molecular interaction potential," J. Chem. Phys. 124, 224318 (2006).

${ }^{51}$ K. Suma, Y. Sumiyoshi, and Y. Endo, "The Rotational Spectrum of the WaterHydroperoxy Radical $\left(\mathrm{H}_{2} \mathrm{O}-\mathrm{HO}_{2}\right)$ Complex," Science 311, 1278-1281 (2006).

${ }^{52}$ Y. Kalugina, F. Lique, and J. Kłos, "Hyperfine collisional rate coefficients of CN with $\mathrm{H}_{2}(j=0)$," Mon. Not. R. Astron. Soc. 422, 812-818 (2012).

${ }^{53}$ B. Yang, X. H. Wang, P. C. Stancil, J. M. Bowman, N. Balakrishnan, and R. C. Forrey, "Full-dimensional quantum dynamics of rovibrationally inelastic scattering between CN and $\mathrm{H}_{2}, "$ J. Chem. Phys. 145, 224307 (2016).

${ }^{54}$ I. D. Petsalakis, D. Tzeli, and G. Theodorakopoulos, "Theoretical study on the electronic states of NaLi," J. Chem. Phys. 129, 054306 (2008).

${ }^{55}$ P. J. Bertoncini, G. Das, and A. C. Wahl, "Theoretical Study of the ${ }^{1} \Sigma^{+},{ }^{3} \Sigma^{+},{ }^{3} \Pi,{ }^{1} \Pi$ States of NaLi and the ${ }^{2} \Sigma^{+}$State of $\mathrm{NaLi}^{+}, "$ J. Chem. Phys. 52, 5112-5130 (1970).

${ }^{56}$ F. Grein, "The $\mathrm{CO}_{2}-\mathrm{O}_{2}$ van der Waals complex," Comp. Theor. Chem. 1114, 101-105 (2017).

${ }^{57}$ S. V. Ryazantsev, D. A. Tyurin, V. I. Feldman, and L. Khriachtchev, "Spectroscopic characterization of the complex of vinyl radical and carbon dioxide: Matrix isolation and ab initio study," J. Chem. Phys. 147, 184301 (2017). 
${ }^{58}$ M. Przybytek and B. Jeziorski, "Bounds for the scattering length of spin-polarized helium from high-accuracy electronic structure calculations," J. Chem. Phys. 123, 134315 (2005).

${ }^{59}$ M. Przybytek, W. Cencek, B. Jeziorski, and K. Szalewicz, "Pair Potential with Submillikelvin Uncertainties and Nonadiabatic Treatment of the Halo State of the Helium Dimer," Phys. Rev. Lett. 119, 123401 (2017).

${ }^{60}$ S. Grimme, S. Ehrlich, and L. Goerigk, "Effect of the damping function in dispersion corrected density functional theory," J. Comp. Chem. 32, 1456-1465 (2011).

${ }^{61}$ H.-J. Werner, P. J. Knowles, G. Knizia, F. R. Manby, M. Schütz, et al., "Molpro, version 2019.2, a package of ab initio programs," (2019), see https://www.molpro.net.

${ }^{62}$ M. Rittby and R. J. Bartlett, "An open-shell spin-restricted coupled cluster method: application to ionization potentials in nitrogen," J. Chem. Phys. 92, 3033-3036 (1988).

${ }^{63}$ G. Knizia, T. B. Adler, and H.-J. Werner, "Simplified CCSD(T)-F12 methods: Theory and benchmarks," J. Chem. Phys. 130, 054104 (2009).

${ }^{64} \mathrm{O}$. Marchetti and H.-J. Werner, "Accurate Calculations of Intermolecular Interaction Energies Using Explicitly Correlated Coupled Cluster Wave Functions and a DispersionWeighted MP2 Method," J. Phys. Chem. A 113, 11580-11585 (2009).

${ }^{65}$ J. Perdew, K. Burke, and M. Ernzerhof, "Generalized Gradient Approximation Made Simple," Phys. Rev. Lett. 77, 3865-3868 (1996).

${ }^{66} \mathrm{C}$. Adamo and V. Barone, "Toward reliable density functional methods without adjustable parameters: The PBE0 model," J. Chem. Phys. 110, 6158-6170 (1999).

${ }^{67} \mathrm{O}$. Vydrov and T. Van Voorhis, "Nonlocal van der waals density functional: The simpler the better," J. Chem. Phys. 133, 244103 (2010).

${ }^{68}$ Y. Shao, Z. Gan, E. Epifanovsky, A. T. Gilbert, M. Wormit, J. Kussmann, A. W. Lange, A. Behn, J. Deng, X. Feng, D. Ghosh, M. Goldey, P. R. Horn, L. D. Jacobson, I. Kaliman, R. Z. Khaliullin, T. Kuś, A. Landau, J. Liu, E. I. Proynov, Y. M. Rhee, R. M. Richard, M. A. Rohrdanz, R. P. Steele, E. J. Sundstrom, H. L. W. III, P. M. Zimmerman, D. Zuev, B. Albrecht, E. Alguire, B. Austin, G. J. O. Beran, Y. A. Bernard, E. Berquist, K. Brandhorst, K. B. Bravaya, S. T. Brown, D. Casanova, C.-M. Chang, Y. Chen, S. H. Chien, K. D. Closser, D. L. Crittenden, M. Diedenhofen, R. A. D. Jr., H. Do, A. D. Dutoi, R. G. Edgar, S. Fatehi, L. Fusti-Molnar, A. Ghysels, A. GolubevaZadorozhnaya, J. Gomes, M. W. Hanson-Heine, P. H. Harbach, A. W. Hauser, E. G. Hohenstein, Z. C. Holden, T.-C. Jagau, H. Ji, B. Kaduk, K. Khistyaev, J. Kim, J. Kim, 
R. A. King, P. Klunzinger, D. Kosenkov, T. Kowalczyk, C. M. Krauter, K. U. Lao, A. D. Laurent, K. V. Lawler, S. V. Levchenko, C. Y. Lin, F. Liu, E. Livshits, R. C. Lochan, A. Luenser, P. Manohar, S. F. Manzer, S.-P. Mao, N. Mardirossian, A. V. Marenich, S. A. Maurer, N. J. Mayhall, E. Neuscamman, C. M. Oana, R. Olivares-Amaya, D. P. O’Neill, J. A. Parkhill, T. M. Perrine, R. Peverati, A. Prociuk, D. R. Rehn, E. Rosta, N. J. Russ, S. M. Sharada, S. Sharma, D. W. Small, A. Sodt, T. Stein, D. Stück, Y.-C. Su, A. J. Thom, T. Tsuchimochi, V. Vanovschi, L. Vogt, O. Vydrov, T. Wang, M. A. Watson, J. Wenzel, A. White, C. F. Williams, J. Yang, S. Yeganeh, S. R. Yost, Z.-Q. You, I. Y. Zhang, X. Zhang, Y. Zhao, B. R. Brooks, G. K. Chan, D. M. Chipman, C. J. Cramer, W. A. G. III, M. S. Gordon, W. J. Hehre, A. Klamt, H. F. S. III, M. W. Schmidt, C. D. Sherrill, D. G. Truhlar, A. Warshel, X. Xu, A. Aspuru-Guzik, R. Baer, A. T. Bell, N. A. Besley, J.-D. Chai, A. Dreuw, B. D. Dunietz, T. R. Furlani, S. R. Gwaltney, C.-P. Hsu, Y. Jung, J. Kong, D. S. Lambrecht, W. Liang, C. Ochsenfeld, V. A. Rassolov, L. V. Slipchenko, J. E. Subotnik, T. V. Voorhis, J. M. Herbert, A. I. Krylov, P. M. Gill, and M. Head-Gordon, "Advances in molecular quantum chemistry contained in the Q-Chem 4 program package," Mol. Phys. 113, 184-215 (2015).

${ }^{69}$ O. A. Vydrov and G. E. Scuseria, "Assessment of a long-range corrected hybrid functional," J. Chem. Phys. 125, 234109 (2006).

${ }^{70}$ S. Grimme, J. Antony, S. Ehrlich, and H. Krieg, "A consistent and accurate ab initio parametrization of density functional dispersion correction (DFT-D) for the 94 elements H-Pu," J. Chem. Phys. 132, 154104 (2010).

${ }^{71}$ M. J. Frisch, G. W. Trucks, H. B. Schlegel, G. E. Scuseria, M. A. Robb, J. R. Cheeseman, G. Scalmani, V. Barone, G. A. Petersson, H. Nakatsuji, X. Li, M. Caricato, A. V. Marenich, J. Bloino, B. G. Janesko, R. Gomperts, B. Mennucci, H. P. Hratchian, J. V. Ortiz, A. F. Izmaylov, J. L. Sonnenberg, D. Williams-Young, F. Ding, F. Lipparini, F. Egidi, J. Goings, B. Peng, A. Petrone, T. Henderson, D. Ranasinghe, V. G. Zakrzewski, J. Gao, N. Rega, G. Zheng, W. Liang, M. Hada, M. Ehara, K. Toyota, R. Fukuda, J. Hasegawa, M. Ishida, T. Nakajima, Y. Honda, O. Kitao, H. Nakai, T. Vreven, K. Throssell, J. A. Montgomery, Jr., J. E. Peralta, F. Ogliaro, M. J. Bearpark, J. J. Heyd, E. N. Brothers, K. N. Kudin, V. N. Staroverov, T. A. Keith, R. Kobayashi, J. Normand, K. Raghavachari, A. P. Rendell, J. C. Burant, S. S. Iyengar, J. Tomasi, M. Cossi, J. M. Millam, M. Klene, C. Adamo, R. Cammi, J. W. Ochterski, R. L. Martin, K. Morokuma, O. Farkas, J. B. 
Foresman, and D. J. Fox, "Gaussian 16 Revision C.01," (2016), gaussian Inc. Wallingford CT.

${ }^{72}$ F. O. Kannemann and A. D. Becke, "van der Waals Interactions in Density-Functional Theory: Intermolecular Complexes," J. Chem. Theory Comput. 6, 1081-1088 (2010).

${ }^{73}$ A. Otero-de-la Roza and E. R. Johnson, "Non-covalent interactions and thermochemistry using XDM-corrected hybrid and range-separated hybrid density functionals," J. Chem. Phys. 138, 204109 (2013).

${ }^{74}$ R. M. Parrish, L. A. Burns, D. G. A. Smith, A. C. Simmonett, A. E. DePrince, E. G. Hohenstein, U. Bozkaya, A. Y. Sokolov, R. Di Remigio, R. M. Richard, J. F. Gonthier, A. M. James, H. R. McAlexander, A. Kumar, M. Saitow, X. Wang, B. P. Pritchard, P. Verma, H. F. Schaefer, K. Patkowski, R. A. King, E. F. Valeev, F. A. Evangelista, J. M. Turney, T. D. Crawford, and C. D. Sherrill, "Psi4 1.1: An open-source electronic structure program emphasizing automation, advanced libraries, and interoperability," J. Chem. Theory Comput. 13, 3185-3197 (2017).

${ }^{75}$ A. Halkier, T. Helgaker, P. Jørgensen, W. Klopper, H. Koch, J. Olsen, and A. K. Wilson, "Basis-set convergence in correlated calculations on $\mathrm{Ne}, \mathrm{N}_{2}$, and $\mathrm{H}_{2} \mathrm{O}$," Chem. Phys. Lett. 286, $243-252$ (1998).

${ }^{76}$ A. Halkier, W. Klopper, T. Helgaker, P. Jørgensen, and P. R. Taylor, "Basis set convergence of the interaction energy of hydrogen-bonded complexes," J. Chem. Phys. 111, 9157-9167 (1999).

${ }^{77}$ T. H. Dunning, "Gaussian basis sets for use in correlated molecular calculations. I. The atoms boron through neon and hydrogen," J. Chem. Phys. 90, 1007-1023 (1989).

${ }^{78}$ R. A. Kendall, T. H. Dunning, and R. J. Harrison, "Electron affinities of the first-row atoms revisited. systematic basis sets and wave functions," J. Chem. Phys. 96, 6796-6806 (1992).

${ }^{79}$ D. E. Woon and T. H. Dunning, "Gaussian basis sets for use in correlated molecular calculations. IV. Calculation of static electrical response properties," J. Chem. Phys. 100, 2975-2988 (1994).

${ }^{80} \mathrm{~F}$. Weigend, "A fully direct RI-HF algorithm: Implementation, optimised auxiliary basis sets, demonstration of accuracy and efficiency," Phys. Chem. Chem. Phys. 4, 4285-4291 (2002). 
${ }^{81}$ F. Weigend, A. Köhn, and C. Hättig, "Efficient use of the correlation consistent basis sets in resolution of the identity MP2 calculations," J. Chem. Phys. 116, 3175-3183 (2002).

${ }^{82} \mathrm{C}$. Hättig, "Optimization of auxiliary basis sets for RI-MP2 and RI-CC2 calculations: Core-valence and quintuple- $\zeta$ basis sets for H to Ar and QZVPP basis sets for Li to Kr," Phys. Chem. Chem. Phys. 7, 59-66 (2005).

${ }^{83}$ S. Boys and F. Bernardi, "The calculation of small molecular interactions by the differences of separate total energies. Some procedures with reduced errors," Mol. Phys. 19, 553-566 (2006).

${ }^{84}$ M. Kodrycka and K. Patkowski, "Platinum, gold, and silver standards of intermolecular interaction energy calculations," J. Chem. Phys. 151, 070901 (2019).

${ }^{85}$ J. ŘRezáč, L. Šimová, and P. Hobza, "CCSD[T] Describes Noncovalent Interactions Better than the CCSD(T), CCSD(TQ), and CCSDT Methods." J. Chem. Theory Comput. 9, 364-9 (2013).

${ }^{86}$ J. D. Watts, J. Gauss, and R. J. Bartlett, "Coupled-cluster methods with noniterative triple excitations for restricted open-shell Hartree-Fock and other general single determinant reference functions. Energies and analytical gradients," J. Chem. Phys. 98, 87188733 (1993).

${ }^{87}$ P. J. Knowles, C. Hampel, and H.-J. Werner, "Erratum: "Coupled cluster theory for high spin, open shell reference wave functions" [ J. Chem. Phys. 99, 5219 (1993)]," J. Chem. Phys. 112, 3106-3107 (2000).

${ }^{88}$ P. G. Szalay and J. Gauss, "Spin-restricted open-shell coupled-cluster theory," J. Chem. Phys. 107, 9028-9038 (1997).

${ }^{89}$ M. Heckert, O. Heun, J. Gauss, and P. G. Szalay, "Towards a spin-adapted coupled-cluster theory for high-spin open-shell states," J. Chem. Phys. 124, 124105 (2006).

${ }^{90}$ P. Neogrády, M. Urban, and I. Hubač, "Spin adapted restricted Hartree-Fock reference coupled cluster theory for open shell systems," J. Chem. Phys. , 3706-3716 (1994).

${ }^{91}$ J. F. Stanton, "On the extent of spin contamination in open-shell coupled-cluster wave functions," J. Chem. Phys. 101, 371-374 (1994).

${ }^{92}$ P. A. Pieniazek, S. E. Bradforth, and A. I. Krylov, "Spectroscopy of the cyano radical in an aqueous environment," J. Phys. Chem. A 110, 4854-4865 (2006).

${ }^{93}$ A. S. Menon and L. Radom, "Consequences of Spin Contamination in Unrestricted Calculations on Open-Shell Species: Effect of Hartree-Fock and Møller-Plesset Contributions 
in Hybrid and Double-Hybrid Density Functional Theory Approaches," J. Phys. Chem. A 112, 13225-13230 (2008).

${ }^{94}$ G. Chałasiński and M. Szczęśniak, "On the connection between the supermolecular MøllerPlesset treatment of the interaction energy and the perturbation theory of intermolecular forces," Mol. Phys. 63, 205-224 (1988).

${ }^{95}$ P. S. Żuchowski, B. Bussery-Honvault, R. Moszynski, and B. Jeziorski, "Dispersion interaction of high-spin open-shell complexes in the random phase approximation," J. Chem. Phys. 119, 10497-10511 (2003).

${ }^{96}$ S. M. Cybulski and M. L. Lytle, "The origin of deficiency of the supermolecule secondorder Møller-Plesset approach for evaluating interaction energies," J. Chem. Phys. 127, 141102 (2007).

${ }^{97}$ A. Heßelmann, "Improved supermolecular second order Møller-Plesset intermolecular interaction energies using time-dependent density functional response theory," J. Chem. Phys. 128, 144112 (2008).

${ }^{98}$ M. Pitoňák and A. Heßelmann, "Accurate Intermolecular Interaction Energies from a Combination of MP2 and TDDFT Response Theory," J. Chem. Theory Comput. 6, 168$178(2010)$.

${ }^{99}$ S. Grimme, "Improved second-order Møller-Plesset perturbation theory by separate scaling of parallel- and antiparallel-spin pair correlation energies," J. Chem. Phys. 118, 90959102 (2003).

${ }^{100}$ R. F. Fink, "Spin-component-scaled møller-plesset (scs-mp) perturbation theory: A generalization of the mp approach with improved properties," J. Chem. Phys. 133, 174113 (2010).

${ }^{101}$ S. Grimme, L. Goerigk, and R. F. Fink, "Spin-component-scaled electron correlation methods," Wiley Interdiscip. Rev.: Comput. Mol. Sci. 2, 886-906 (2012).

${ }^{102} \mathrm{O}$. Marchetti and H.-J. Werner, "Accurate calculations of intermolecular interaction energies using explicitly correlated wave functions," Phys. Chem. Chem. Phys. 10, 3400-3409 (2008).

${ }^{103}$ J. R. Lane and H. G. Kjaergaard, "Explicitly correlated intermolecular distances and interaction energies of hydrogen bonded complexes," J. Chem. Phys. 131, 034307 (2009). ${ }^{104}$ D. A. Sirianni, L. A. Burns, and C. D. Sherrill, "Comparison of explicitly correlated methods for computing high-accuracy benchmark energies for noncovalent interactions," 
J. Chem. Theory Comput. 13, 86-99 (2017).

${ }^{105}$ F. O. Kannemann and A. D. Becke, "Van der Waals Interactions in Density-Functional Theory: Rare-Gas Diatomics," J. Chem. Theory Comput. 5, 719-727 (2009).

${ }^{106}$ S. Grimme, A. Hansen, J. G. Brandenburg, and C. Bannwarth, "Dispersion-corrected mean-field electronic structure methods," Chem. Rev. 116, 5105-5154 (2016).

${ }^{107}$ T. M. Henderson, B. G. Janesko, and G. E. Scuseria, "Generalized gradient approximation model exchange holes for range-separated hybrids," J. Chem. Phys. 128, 194105 (2008).

${ }^{108}$ R. Podeszwa and K. Szalewicz, "Communication: Density functional theory overcomes the failure of predicting intermolecular interaction energies," J. Chem. Phys. 136, 161102 (2012).

${ }^{109}$ T. Thonhauser, S. Zuluaga, C. Arter, K. Berland, E. Schröder, and P. Hyldgaard, "Spin signature of nonlocal correlation binding in metal-organic frameworks," Phys. Rev. Lett. 115, $136402(2015)$.

${ }^{110}$ M. Dion, H. Rydberg, E. Schröder, D. Langreth, and B. Lundqvist, "Van der Waals density functional for general geometries," Phys. Rev. Lett. 92, 246401 (2004).

${ }^{111}$ K. Lee, E. Murray, L. Kong, B. Lundqvist, and D. Langreth, "Higher-accuracy van der waals density functional," Phys. Rev. B 82, 081101 (2010).

${ }^{112} \mathrm{O}$. Vydrov and T. Van Voorhis, "Nonlocal van der waals density functional: The simpler the better," J. Chem. Phys. 133, 244103 (2010).

${ }^{113} \mathrm{~A}$. Becke and E. Johnson, "A unified density-functional treatment of dynamical, nondynamical, and dispersion correlations," J. Chem. Phys. 127, 124108-124108 (2007).

${ }^{114}$ A. D. Becke and E. R. Johnson, "Exchange-hole dipole moment and the dispersion interaction revisited," J. Chem. Phys. 127, 154108 (2007).

${ }^{115}$ M. Warehime, E. R. Johnson, and J. Klos, "New XDM-corrected potential energy surfaces for $\operatorname{Ar}-\mathrm{NO}\left(X^{2} \Pi\right)$ : A comparison with $\operatorname{CCSD}(\mathrm{T})$ calculations and experiments," J. Chem. Phys. 142, 024302 (2015).

${ }^{116}$ E. R. Johnson and A. D. Becke, "A post-Hartree-Fock model of intermolecular interactions: Inclusion of higher-order corrections," J. Chem. Phys. 124, 174104 (2006).

${ }^{117}$ M. Shahbaz and K. Szalewicz, "Evaluation of methods for obtaining dispersion energies used in density functional calculations of intermolecular interactions," Theor. Chem. Acc. 138, 25 (2019). 
${ }^{118}$ M. Shahbaz and K. Szalewicz, "Do semilocal density-functional approximations recover dispersion energies at small intermonomer separations?" Phys. Rev. Lett. 121, 113402 (2018). 


\section{Supporting Information:}

\section{Dataset of noncovalent intermolecular interaction energy curves for 24 small high-spin open-shell dimers}

Katarzyna Madajczyk, ${ }^{\dagger}$ Piotr S. Żuchowski, ${ }^{* \dagger}{ }^{\dagger}$ Filip Brzęk, ${ }^{\dagger}$ Łukasz Rajchel, ${ }^{\ddagger}$

Dariusz Kędziera, "Marcin Modrzejewski, ${ }^{\S}$ and Michał Hapka*,\|,§

$\dagger$ Institute of Physics, Faculty of Physics, Astronomy and Informatics, Nicolaus Copernicus University, Grudziadzka 5,Toruń, Poland

$\ddagger$ Institute of Physics, Kazimierz Wielki University, al. Powstańców Wielkopolskich 2, 85-090 Bydgoszcz, Poland

\Department of Chemistry, Nicolaus Copernicus University, 7 Gagarin Street, 87-100 Toruń, Poland

§Faculty of Chemistry, University of Warsaw, ul. L. Pasteura 1, 02-093 Warsaw, Poland ||Department of Chemistry, University of Michigan, Ann Arbor, Michigan 48109, USA

E-mail: pzuch@fizyka.umk.pl; hapka@tiger.chem.uw.edu.pl

SAPT0 calculations The interaction energy at the SAPT0 level of theory is obtained as

$$
E_{\text {int }}^{\mathrm{SAPT0}}=E_{\text {elst }}^{(10)}+E_{\text {exch }}^{(10)}+E_{\text {ind }, \mathrm{r}}^{(20)}+E_{\text {exch-ind,r }}^{(20)}+E_{\text {disp }}^{(20)}+E_{\text {exch-disp }}^{(20)}+\delta_{\mathrm{HF}},
$$


where $E_{\text {elst }}^{(10)}$ and $E_{\text {exch }}^{(10)}$ are first-order electrostatic and exchange interaction energy contributions, respectively; $E_{\text {ind,r }}^{(20)}$ and $E_{\text {disp }}^{(20)}$ are the second-order induction and dispersion energies, respectively; $E_{\text {exch-ind,r }}^{(20)}$ and $E_{\text {exch-disp }}^{(20)}$ are second-order exchange-induction and exchangedispersion energies, respectively. The "r" subscript indicates that orbital relaxation effects are taken into account. The $\delta_{\mathrm{HF}}$ term approximates higher-order induction energy contributions and is defined as

$$
\delta_{\mathrm{HF}}=E_{\mathrm{int}}^{\mathrm{UHF}}-\left(E_{\mathrm{elst}}^{(10)}+E_{\mathrm{exch}}^{(10)}+E_{\mathrm{ind}, \mathrm{r}}^{(20)}+E_{\text {exch-ind,r }}^{(20)}\right)
$$

where $E_{\text {int }}^{\mathrm{UHF}}$ is the UHF interaction energy.

The ternary diagram was obtained by splitting the SAPT0 interaction contributions into: $E_{\text {elst }}^{(10)}, E_{\mathrm{IND}}^{(20)}=E_{\text {ind,r }}^{(20)}+E_{\text {exch-ind,r }}^{(20)}, E_{\text {DISP }}^{(20)}=E_{\text {disp }}^{(20)}+E_{\text {exch-disp }}^{(20)}$. Each energy point was mapped to $(x, y)$ coordinates within the parallelogram, where each vertex corresponds to the interaction of a pure type: $\operatorname{ELST}(+):(-0.5,1.0), \operatorname{IND}(-):(0.5,1.0), \operatorname{ELST}(-):(1.0,0.0)$, and DISP(-): $(0.0,0.0)$. Affiliation to each interaction group was chosen according the following criteria: a) all points within a radius of 0.35 radii from the origin $(0.0,0.0)$ were assigned to the dispersion subset, b) all points within a radius of 0.36 radii from the $(1.0,0.0)$ vertex were assigned as electrostatic species, c) all the remaining points were assigned to the mixed interaction subset. An exception was made for the $\mathrm{C}_{2} \mathrm{H}_{3}-\mathrm{C}_{2} \mathrm{H}_{4}$ dimer-although it is positioned slightly beyond the desired range for the dispersion subset, the dispersion contribution is the dominating stabilizing energy component. Thus, $\mathrm{C}_{2} \mathrm{H}_{3}-\mathrm{C}_{2} \mathrm{H}_{4}$ is included in the dispersion subset. The atypical position of this complex on the ternary diagram results from the repulsive character of the electrostatic energy. 


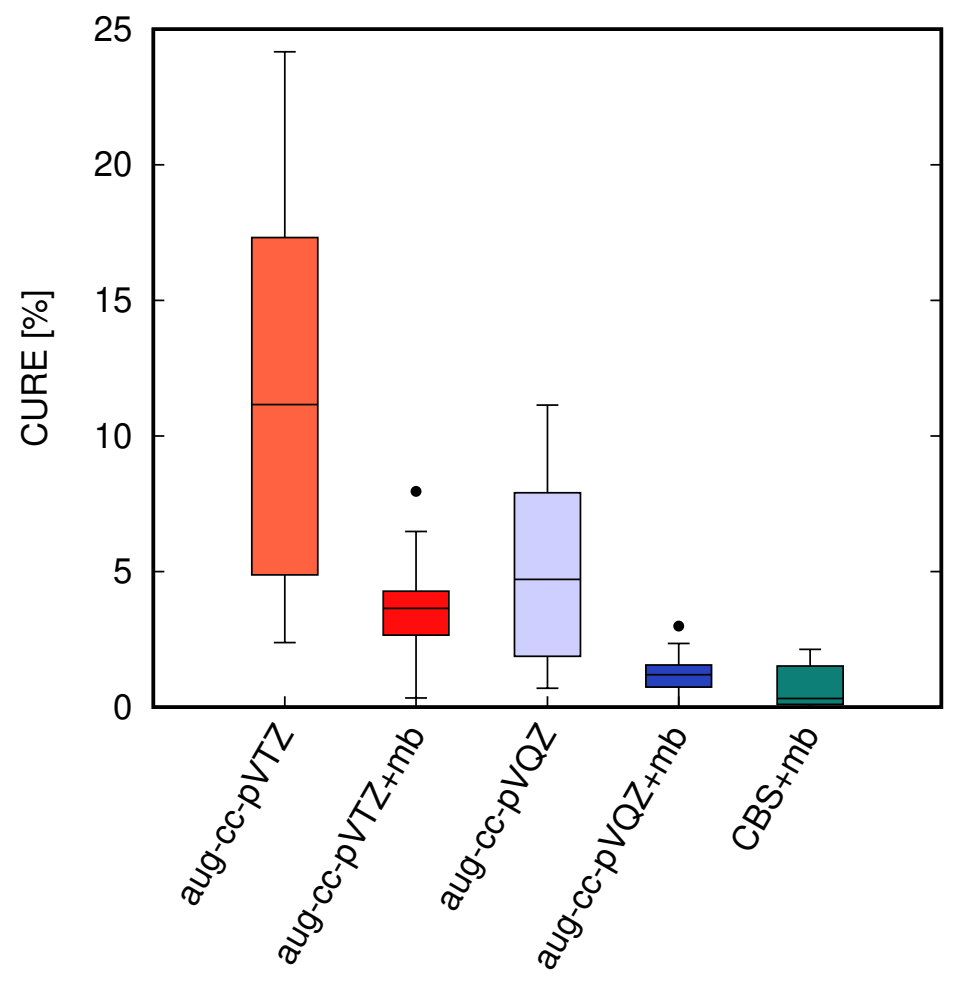

Figure S1: Capped unsigned errors (in percent) of $\operatorname{RCCSD}(\mathrm{T})$ calculations in different basis sets at $R / R_{\mathrm{eq}}=1.0$. CBS denotes the two-point aug-cc-pVQZ $\rightarrow$ aug-cc-pV5Z extrapolation $(\mathrm{mb}=$ midbond functions, see the manuscript for details). Errors given with respect to $\operatorname{RCCSD}(\mathrm{T}) / \mathrm{CBS}$ benchmark.

Table S1: Interaction energies $R=100 R_{\text {eq }}$ computed with RCCSD(T) and its explicitly correlated open-shell variants (in $\mathrm{cm}^{-1}$ ). Both RCCSD and RCSSD $(\mathrm{T})$ calculations were performed in the aug-cc-pV5Z basis set. Calculations with explicitly correlated methods were performed in the orbital aug-cc-pVTZ basis.

\begin{tabular}{lrrrrrr}
\hline$R=100 R_{\text {eq }}$ & \multicolumn{2}{c}{ RCCSD } & \multicolumn{2}{c}{ RCCSD $\left(\mathrm{T}^{* *}\right)$} & RCCSD & RCCSD $(\mathrm{T})$ \\
& $-\mathrm{F} 12 \mathrm{a}$ & $-\mathrm{F} 12 \mathrm{~b}$ & $-\mathrm{F} 12 \mathrm{a}$ & $-\mathrm{F} 12 \mathrm{~b}$ & & \\
\hline $\mathrm{NH}-\mathrm{NH}$ & -0.98 & -0.96 & 0.14 & 0.17 & -1.13 & 0.15 \\
$\mathrm{Li}-\mathrm{O}_{2}$ & -0.57 & -0.56 & 0.33 & 0.34 & -0.66 & 0.42 \\
$\mathrm{O}_{2}-\mathrm{O}_{2}$ & -1.84 & -1.81 & 0.81 & 0.83 & -2.12 & 1.06 \\
\hline
\end{tabular}


Table S2: Spin contamination at UMP2 and UCCSD levels of theory in the equilibrium geometry $\left(R / R_{\text {eq }}=1.0\right)$. Basis set is aug-cc-pV5Z.

\begin{tabular}{|c|c|c|c|c|c|c|}
\hline & mon $\mathrm{A}$ & $\begin{array}{l}\text { UMP2 } \\
\text { mon B }\end{array}$ & dimer & mon $\mathrm{A}$ & $\begin{array}{c}\text { UCCSD } \\
\text { mon B }\end{array}$ & dimer \\
\hline $\mathrm{Na}-\mathrm{Li}$ & 00000 & 00000 & .00000 & .00000 & 0.00000 & 0.00000 \\
\hline $\mathrm{He}-\mathrm{He}^{*}$ & 00000 & 00000 & 00000 & .00000 & 0.00000 & 0.00000 \\
\hline $\mathrm{H}_{2} \mathrm{O}-\mathrm{Na}$ & 0.00000 & 0.00000 & 0.00002 & 0.00000 & 0.00000 & 0.00013 \\
\hline $\mathrm{Li}-\mathrm{NH}_{3}(\mathrm{~lm})$ & 0.00000 & 0.00000 & 0.00002 & 0.00000 & 0.00000 & 0.00005 \\
\hline $\mathrm{Li}-\mathrm{NH}_{3}(\mathrm{gm})$ & 0.00000 & 0.00000 & 0.00003 & 0.00000 & 0.00000 & 0.00020 \\
\hline $\mathrm{C}_{2} \mathrm{H}_{3}-\mathrm{CO}_{2}$ & 0.00384 & 0.00000 & 0.00150 & 0.00752 & 0.00000 & 0.00352 \\
\hline $\mathrm{H}_{2} \mathrm{O}-\mathrm{O}_{2} \mathrm{H}$ & 0.00000 & 0.00392 & 0.00263 & 0.00000 & 0.00347 & 0.00257 \\
\hline $\mathrm{H}_{2} \mathrm{O}-\mathrm{OH}$ & 0.00000 & 0.00394 & 0.00168 & 0.00000 & 0.00173 & 0.00083 \\
\hline $\mathrm{CH}_{2} \mathrm{O}-\mathrm{NH}_{2}$ & 0.00000 & 0.00442 & 0.00144 & 0.00000 & 0.00188 & 0.00073 \\
\hline $\mathrm{HF}-\mathrm{CO}^{+}$ & 0.00000 & 0.00628 & 0.00419 & 0.00000 & 0.01060 & 0.00922 \\
\hline $\mathrm{CO}_{2}-\mathrm{O}_{2}$ & 0.00000 & 0.00676 & 0.00220 & 0.00000 & 0.00293 & 0.00095 \\
\hline $\mathrm{CN}-\mathrm{Ar}$ & 0.00724 & 0.00000 & 0.00479 & 0.00886 & 0.00000 & 0.00648 \\
\hline $\mathrm{CN}-\mathrm{H}_{2}$ & 0.00724 & 0.00000 & 0.00667 & 0.00886 & 0.00000 & 0.00811 \\
\hline $\mathrm{CN}-\mathrm{He}$ & 0.00724 & 0.00000 & 0.00693 & 0.00886 & 0.00000 & 0.00851 \\
\hline $\mathrm{H}_{2} \mathrm{O}-\mathrm{O}_{2}$ & 0.00000 & 0.00876 & 0.00561 & 0.00000 & 0.00362 & 0.00222 \\
\hline $\mathrm{O}_{2}-\mathrm{N}_{2}$ & 0.00932 & 0.00000 & 0.00483 & 0.00384 & 0.00000 & 0.00187 \\
\hline $\mathrm{O}_{2}-\mathrm{H}_{2}$ & 0.00933 & 0.00000 & 0.00858 & 0.00384 & 0.00000 & 0.00333 \\
\hline $\mathrm{O}_{2}-\mathrm{O}_{2}$ & 0.00933 & 0.00933 & 0.00913 & 0.00384 & 0.00384 & 0.00384 \\
\hline $\mathrm{Li}-\mathrm{O}_{2}$ & 0.00936 & 0.00000 & 0.00932 & 0.00385 & 0.00000 & 0.00386 \\
\hline $\mathrm{NH}-\mathrm{He}$ & 0.01182 & 0.00000 & 0.01044 & 0.00316 & 0.00000 & 0.00277 \\
\hline $\mathrm{NH}-\mathrm{Ar}$ & 0.01182 & 0.00000 & 0.00471 & 0.00316 & 0.00000 & 0.00141 \\
\hline $\mathrm{NH}-\mathrm{NH}$ & 0.01184 & 0.01184 & 0.01155 & 0.00315 & 0.00316 & 0.00309 \\
\hline $\mathrm{C}_{2} \mathrm{H}_{3}-\mathrm{C}_{2} \mathrm{H}_{4}$ & 0.24720 & 0.00000 & 0.25280 & 0.00911 & 0.00000 & 0.00466 \\
\hline
\end{tabular}




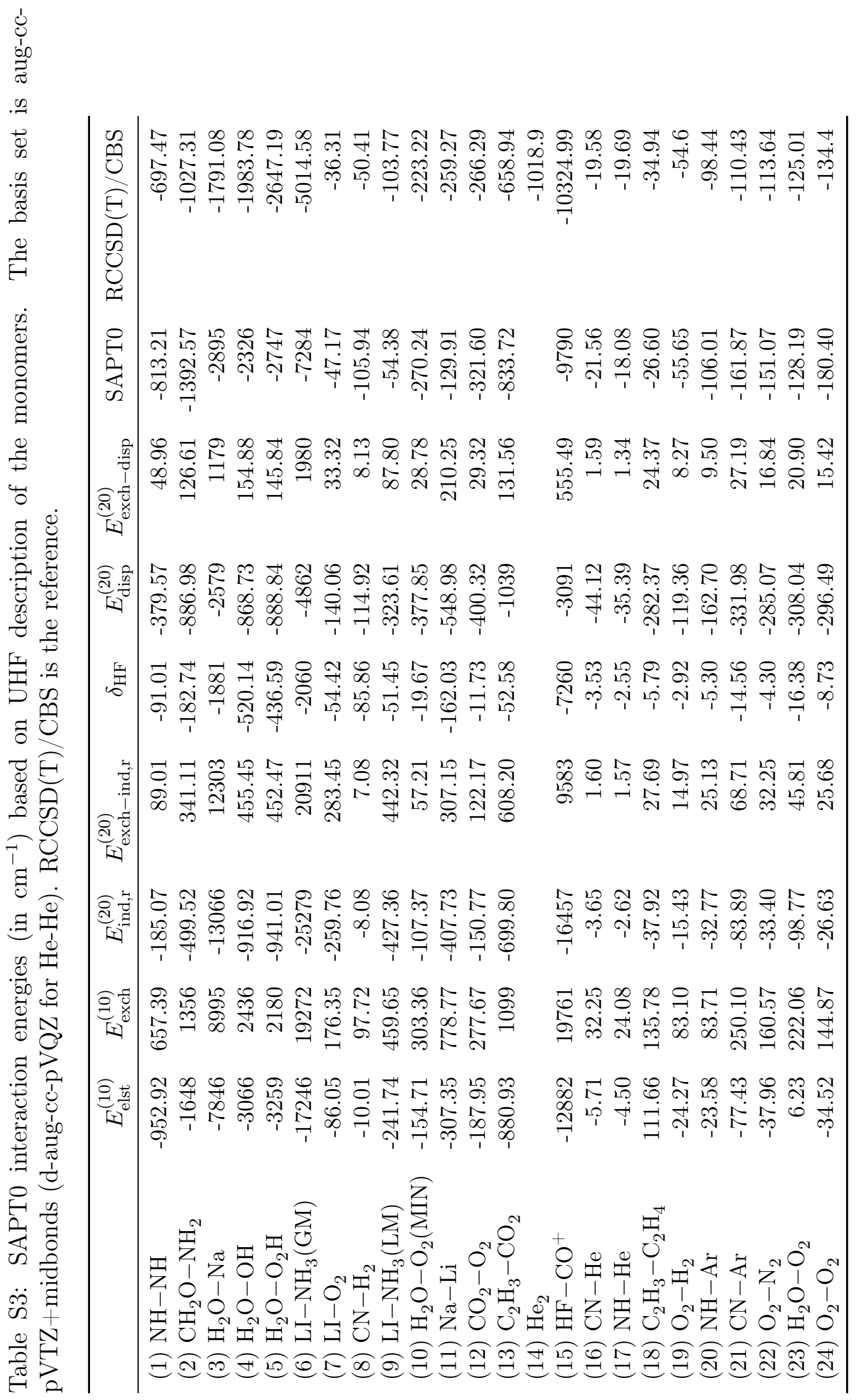




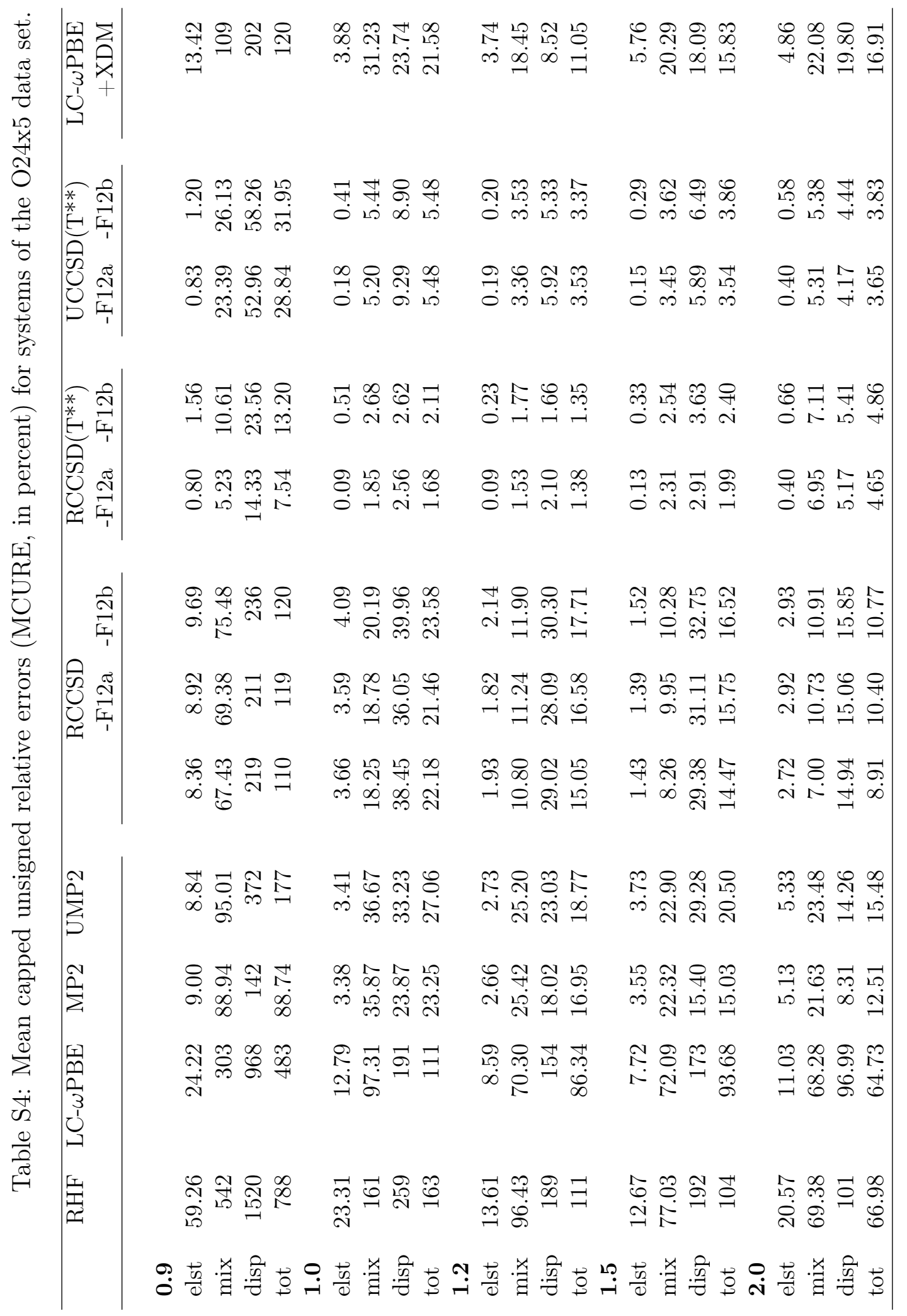




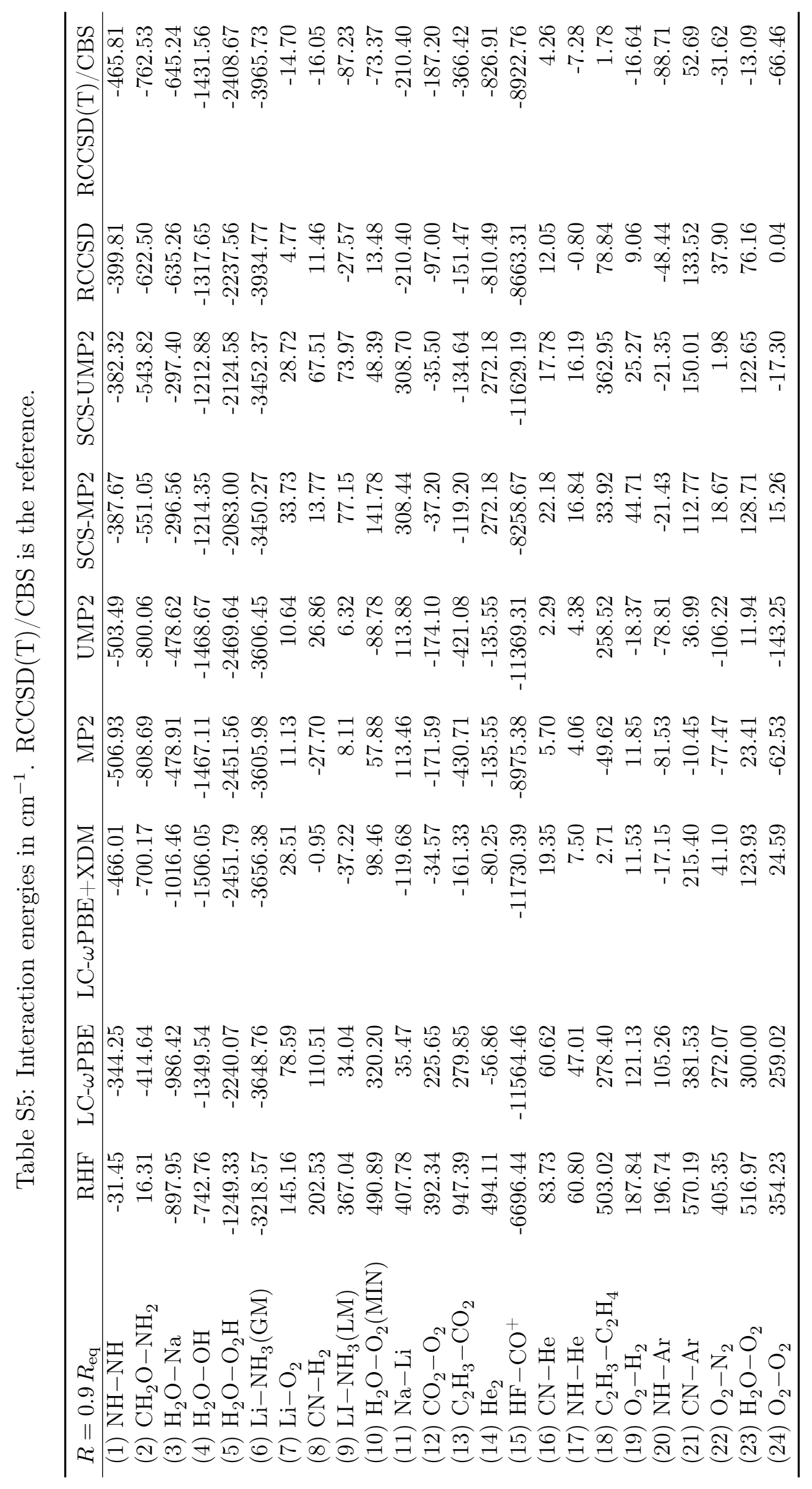




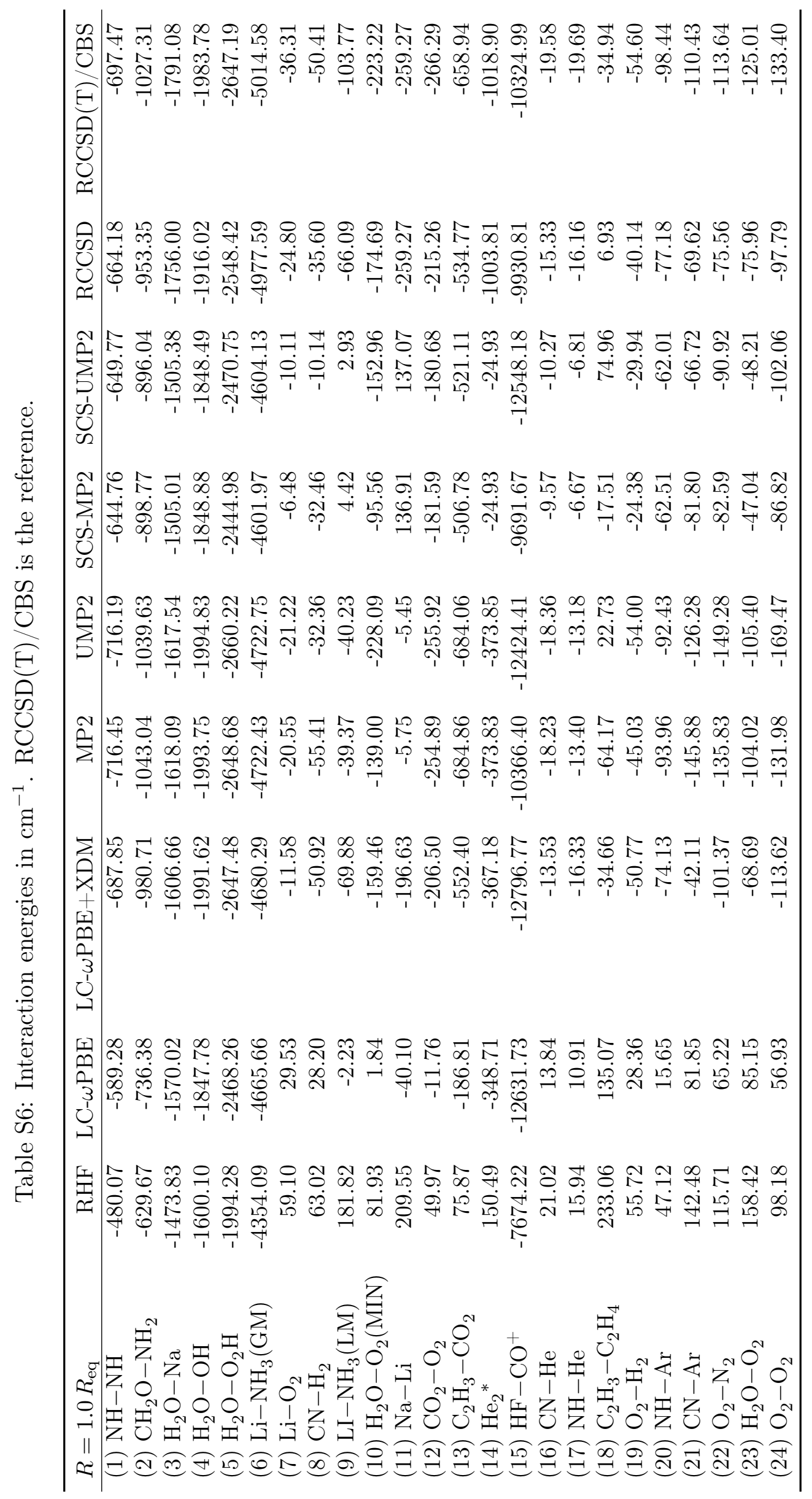




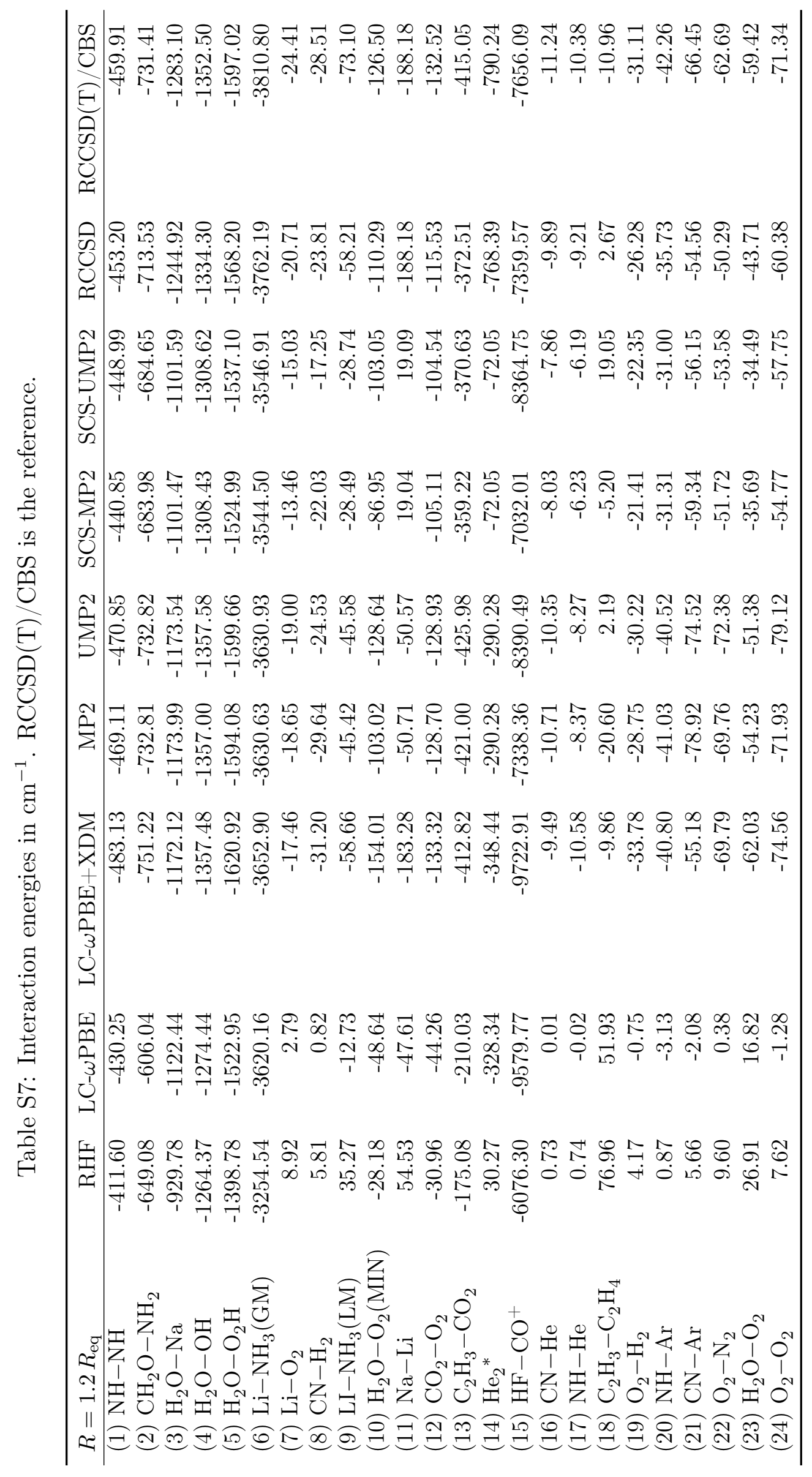




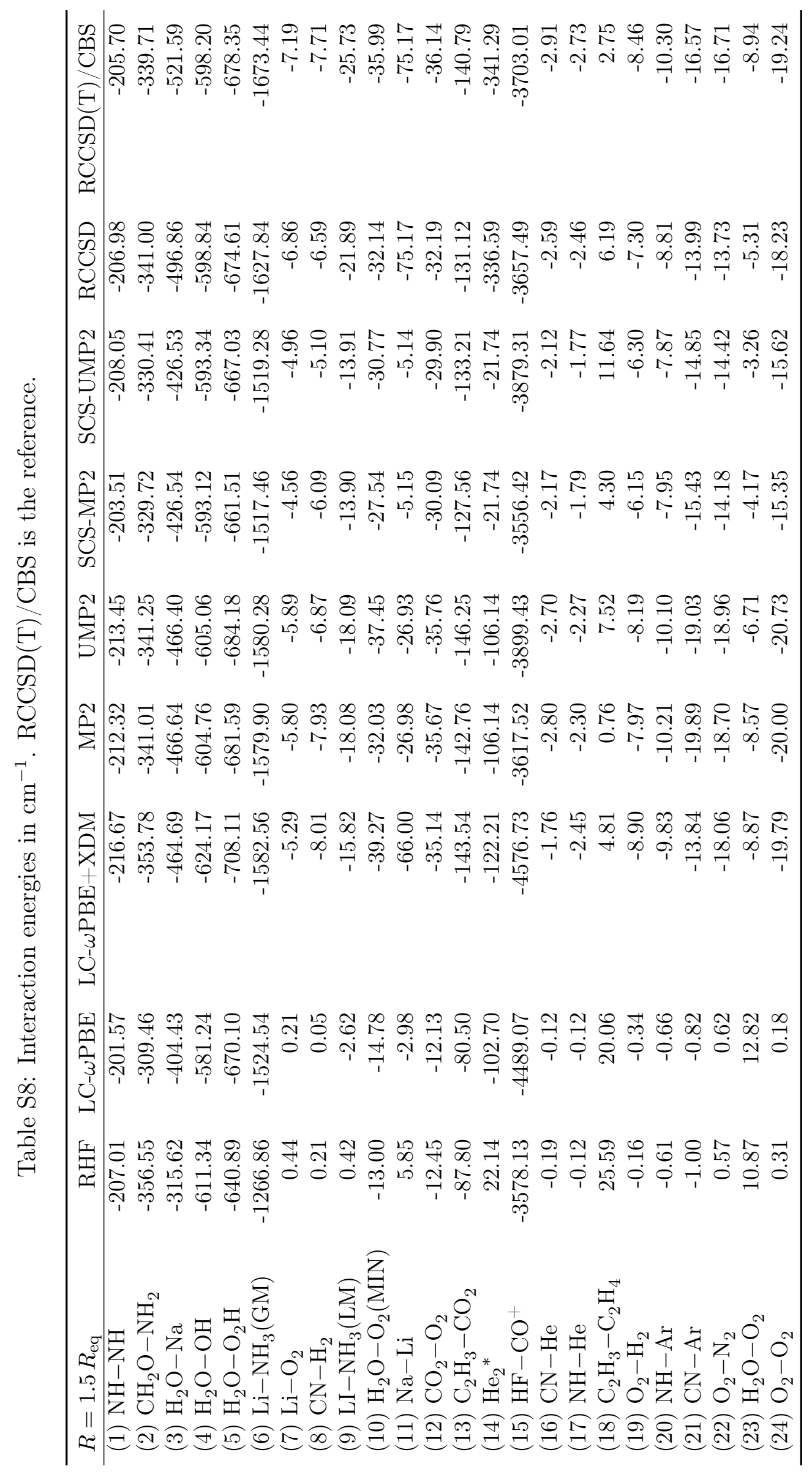




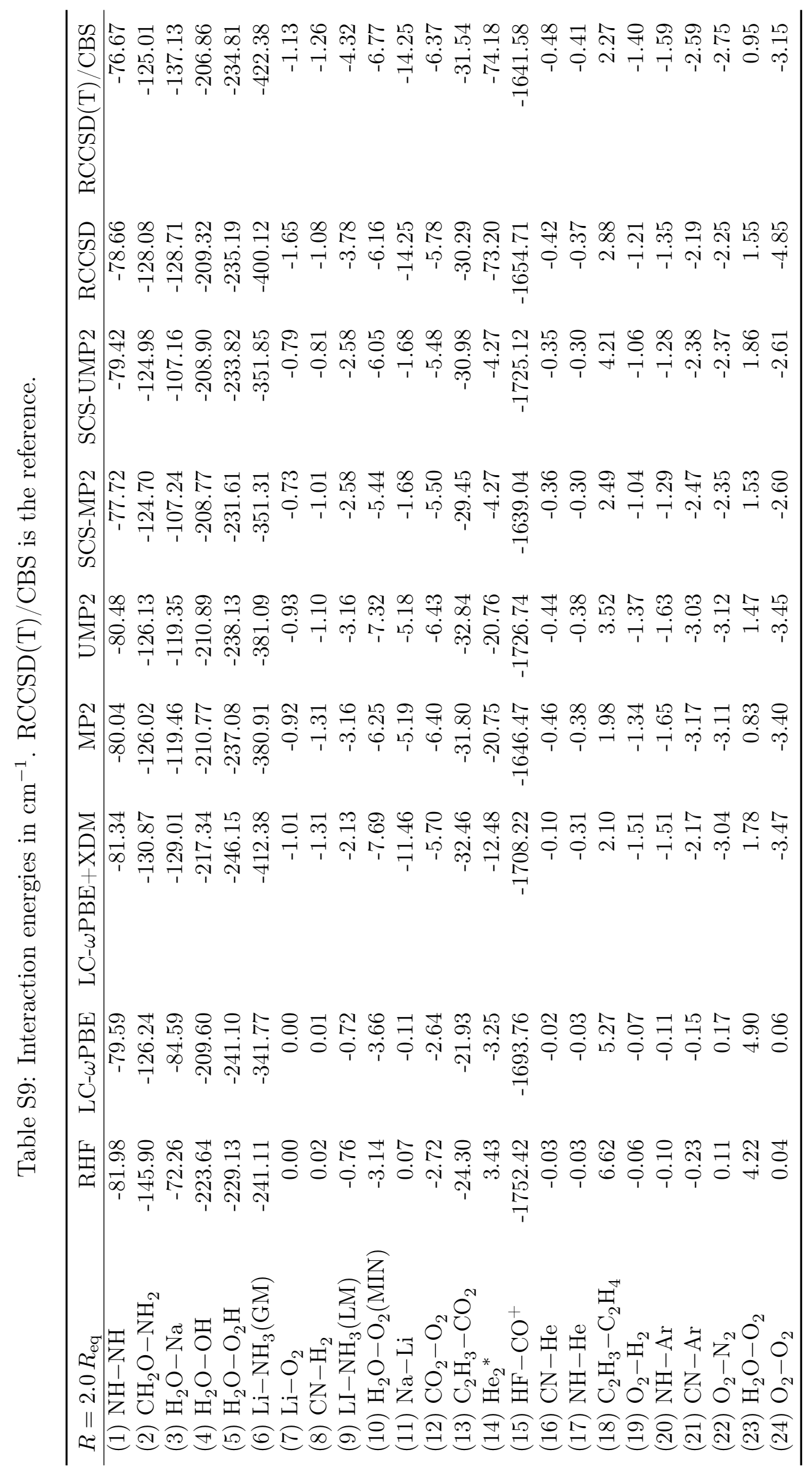




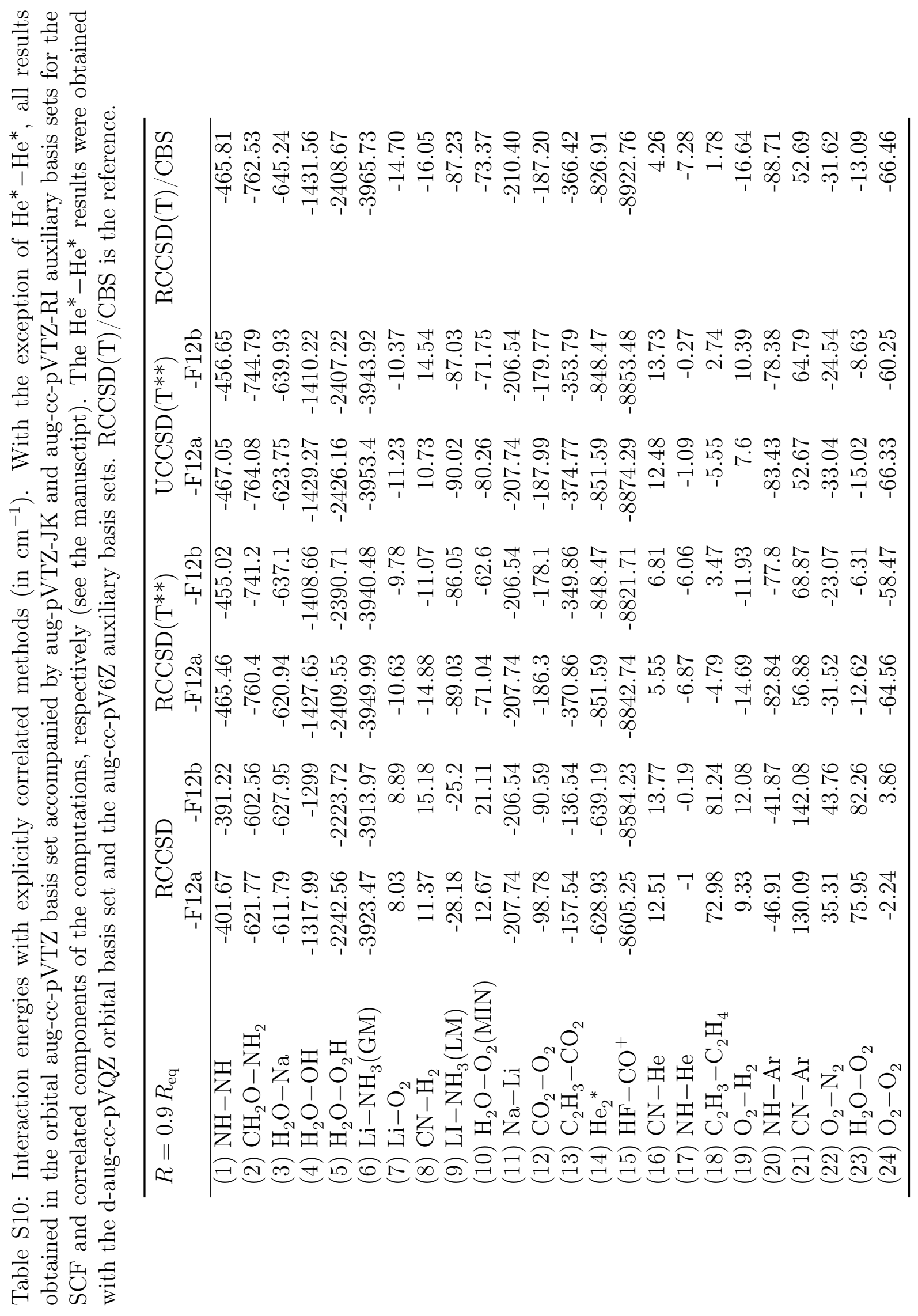




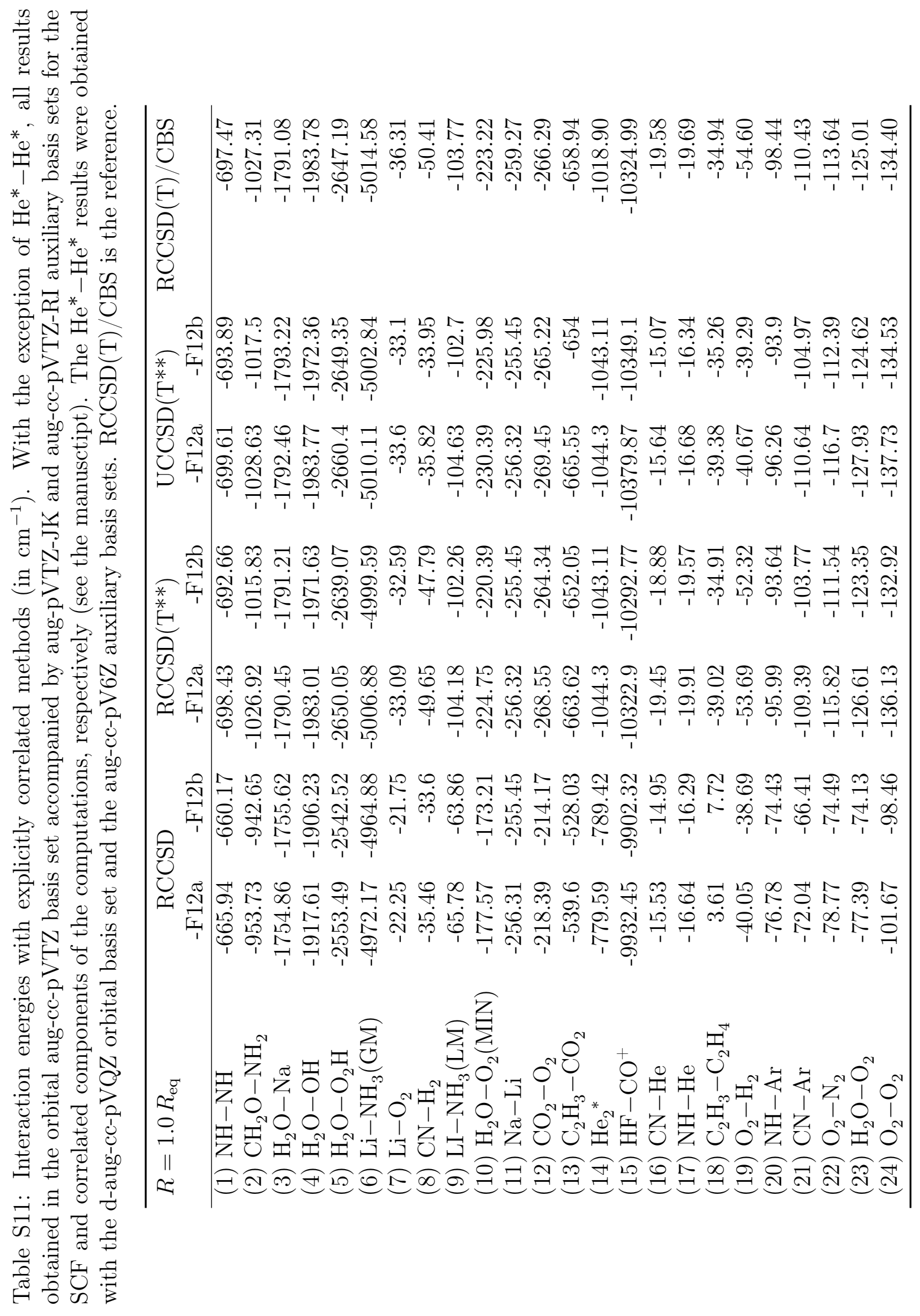




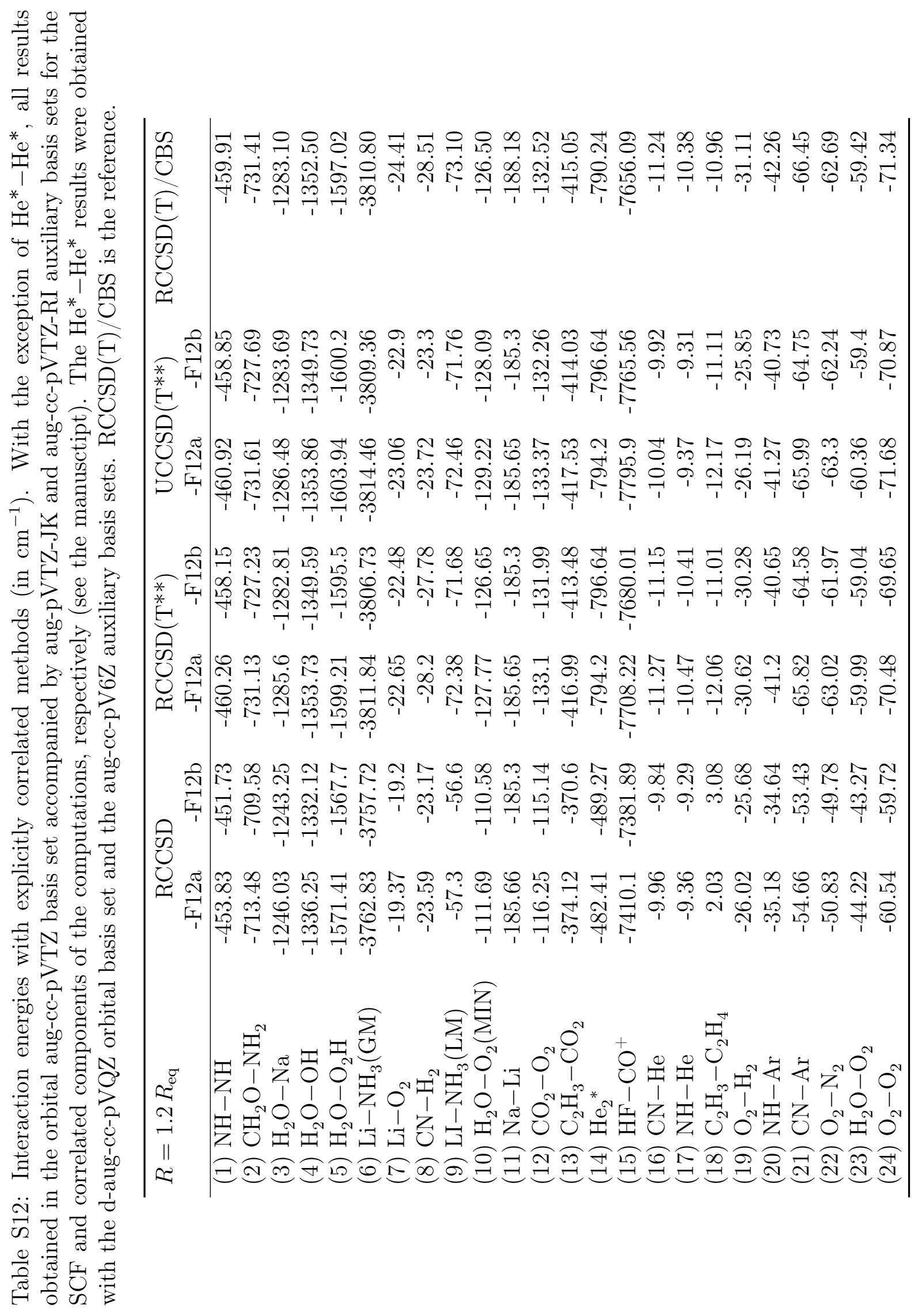




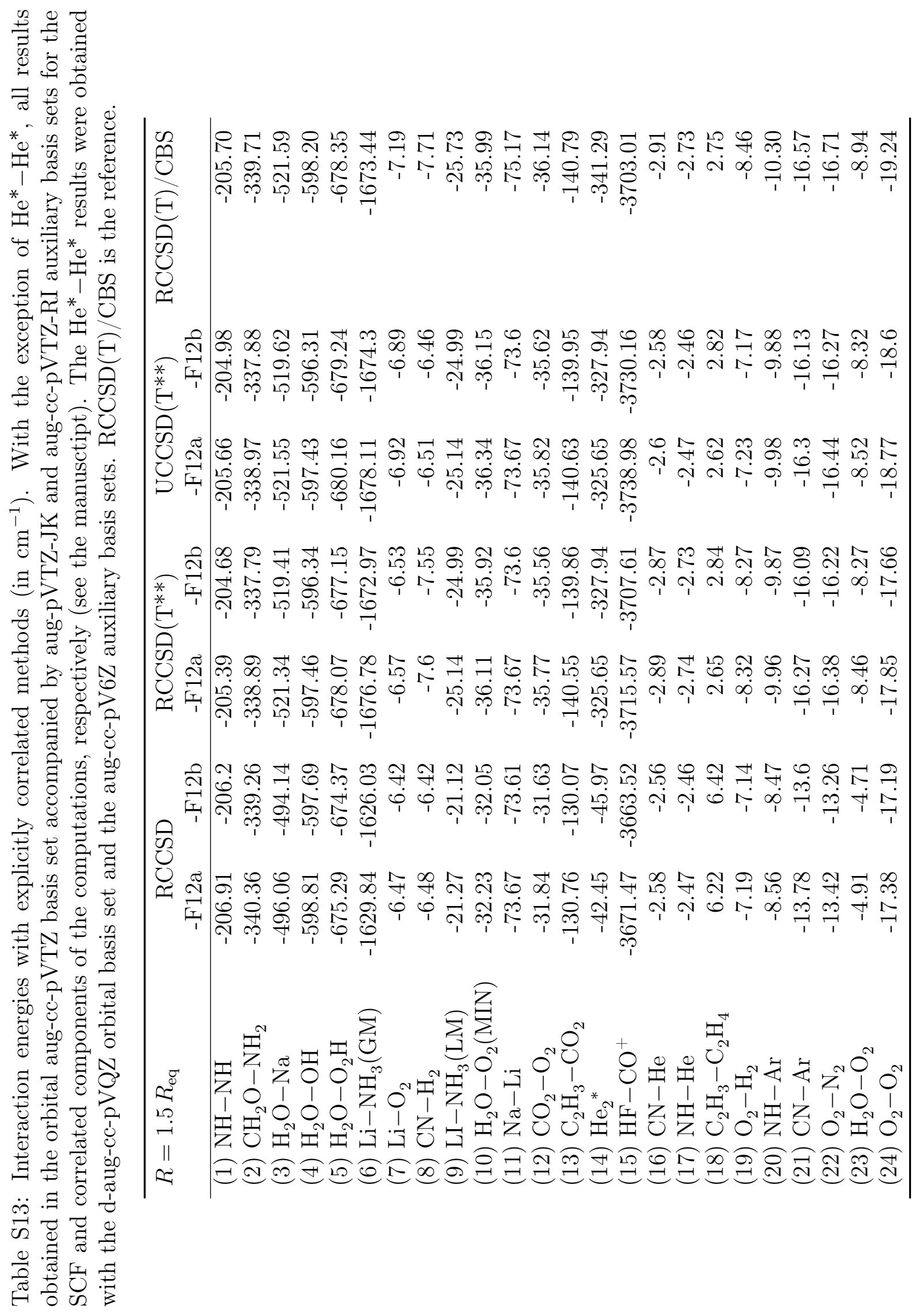




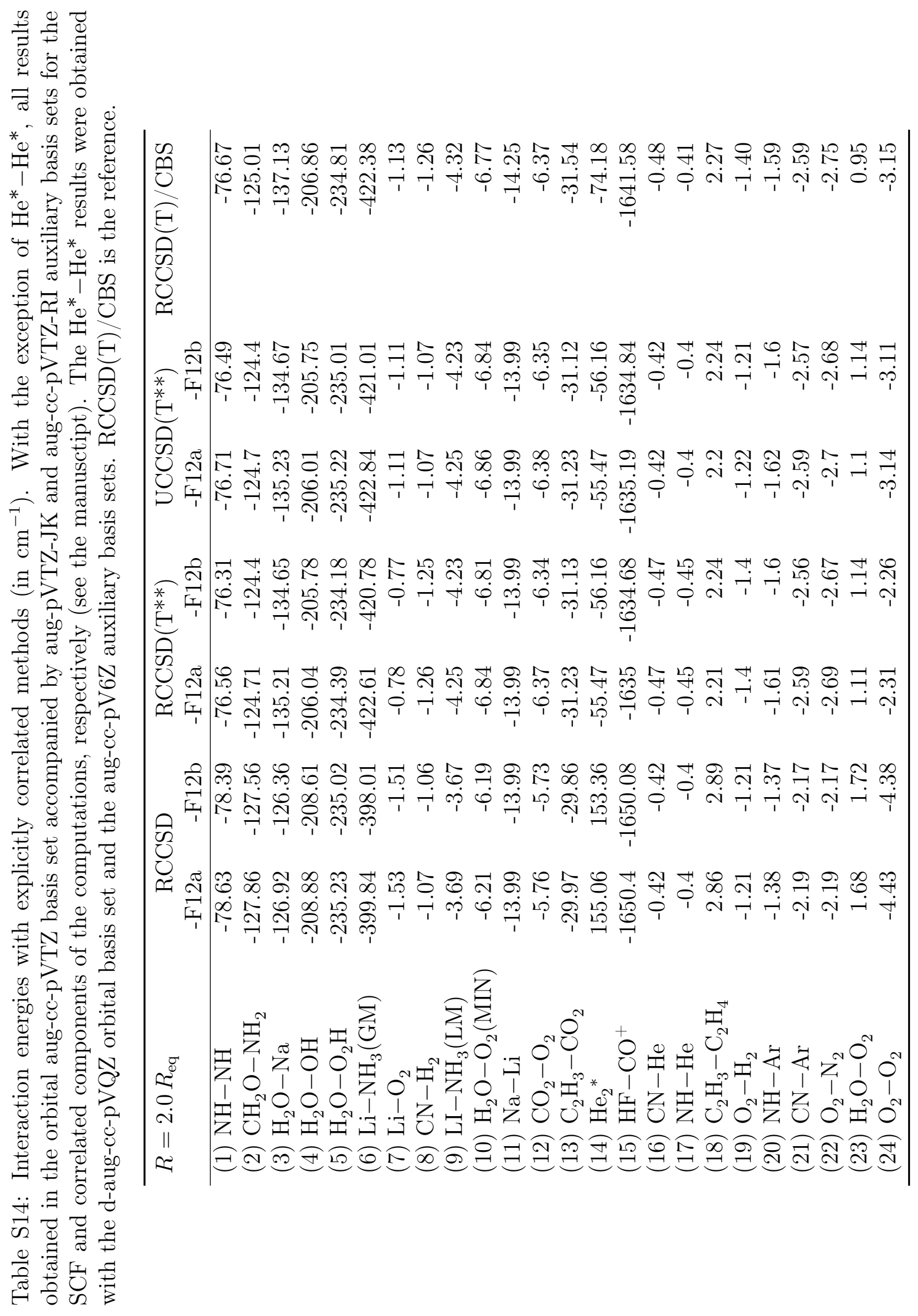


Table S15: Comparison of RCCSD(T) and $\operatorname{UCCSD}(\mathrm{T})$ interaction energies obtained in the aug-cc-pVTZ basis set with midbond function (see text for details).

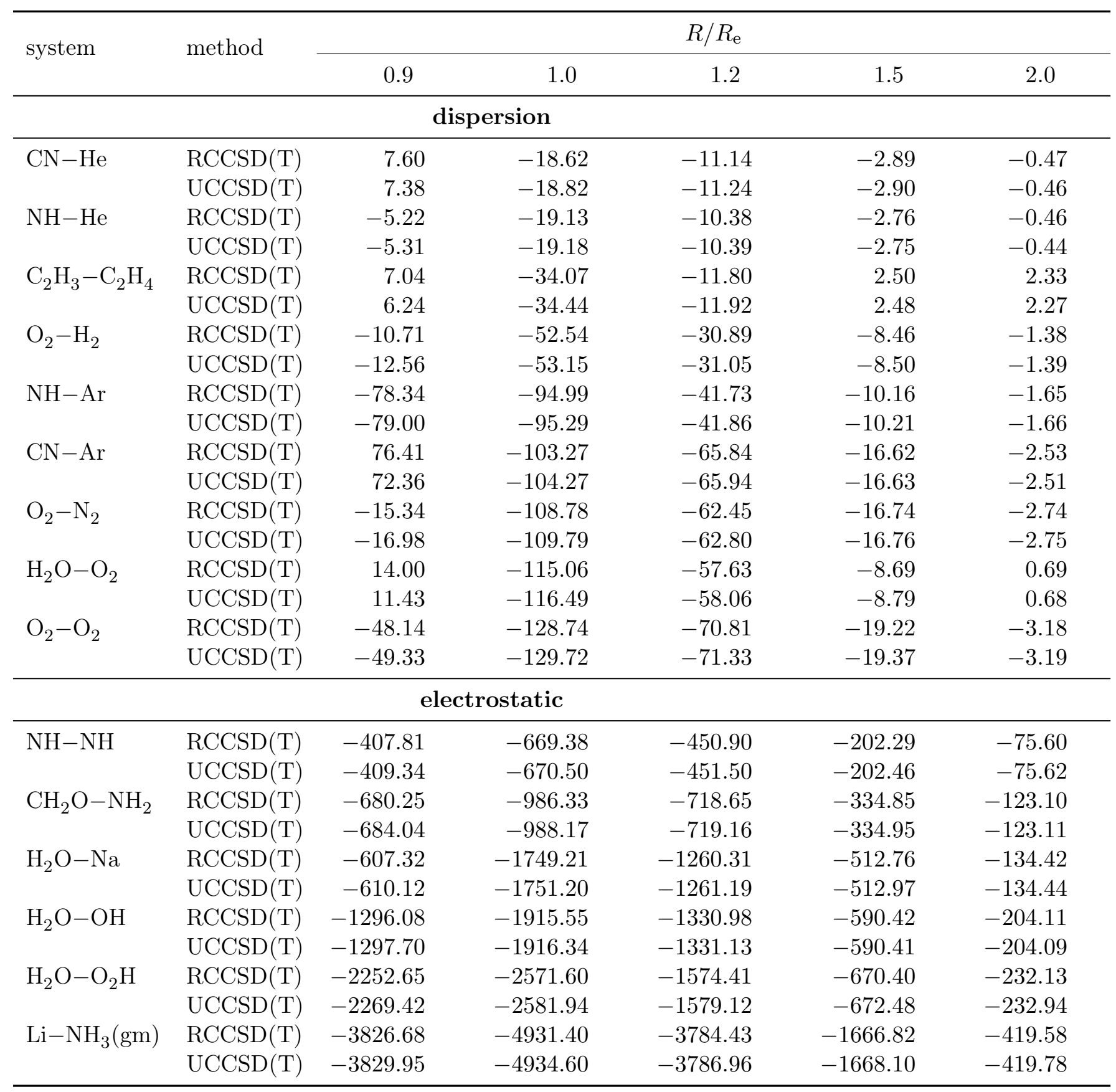




\begin{tabular}{lllccrc}
\hline \multicolumn{5}{c}{ mixed } \\
$\mathrm{Li}-\mathrm{O}_{2}$ & $\mathrm{RCCSD}(\mathrm{T})$ & -11.12 & -34.25 & -23.72 & -7.07 & -1.11 \\
& $\mathrm{UCCSD}(\mathrm{T})$ & -11.45 & -34.48 & -23.82 & -7.09 & -1.12 \\
$\mathrm{CN}-\mathrm{H}_{2}$ & $\mathrm{RCCSD}(\mathrm{T})$ & -10.64 & -48.26 & -28.25 & -7.71 & -1.26 \\
& $\mathrm{UCCSD}(\mathrm{T})$ & -11.39 & -48.68 & -28.37 & -7.70 & -1.21 \\
$\mathrm{Li}-\mathrm{NH}_{3}(\mathrm{~lm})$ & $\mathrm{RCCSD}(\mathrm{T})$ & -82.89 & -100.79 & -71.75 & -25.30 & -4.27 \\
& $\mathrm{UCCSD}(\mathrm{T})$ & -83.89 & -101.24 & -71.82 & -25.31 & -4.27 \\
$\mathrm{H}_{2} \mathrm{O}-\mathrm{O}_{2}(\mathrm{gm})$ & $\mathrm{RCCSD}(\mathrm{T})$ & -43.86 & -213.72 & -125.44 & -35.87 & -6.61 \\
& $\mathrm{UCCSD}(\mathrm{T})$ & -52.82 & -219.18 & -126.93 & -36.13 & -6.63 \\
$\mathrm{Na}^{-} \mathrm{Li}$ & $\mathrm{RCCSD}(\mathrm{T})$ & -206.77 & -256.39 & -186.36 & -74.30 & -14.12 \\
& $\mathrm{UCCSD}(\mathrm{T})$ & -206.77 & -256.40 & -186.36 & -74.30 & -14.12 \\
$\mathrm{CO}_{2}-\mathrm{O}_{2}$ & $\mathrm{RCCSD}(\mathrm{T})$ & -160.24 & -258.06 & -131.87 & -36.16 & -6.38 \\
& $\mathrm{UCCSD}(\mathrm{T})$ & -162.05 & -259.04 & -132.18 & -36.18 & -6.39 \\
$\mathrm{C}_{2} \mathrm{H}_{3}-\mathrm{CO}_{2}$ & $\mathrm{RCCSD}(\mathrm{T})$ & -293.74 & -628.70 & -409.56 & -139.87 & -31.11 \\
& $\mathrm{UCCSD}(\mathrm{T})$ & -297.73 & -630.68 & -410.14 & -139.97 & -31.10 \\
$\mathrm{He}_{2}{ }^{*}$ & $\mathrm{RCCSD}(\mathrm{T})$ & -832.67 & -1022.40 & -783.37 & -358.79 & -86.10 \\
& $\mathrm{UCCSD}(\mathrm{T})$ & -832.67 & -1022.40 & -783.37 & -358.79 & -86.10 \\
$\mathrm{HF}-\mathrm{CO}^{+}$ & $\mathrm{RCCSD}(\mathrm{T})$ & -8447 & -10078 & -7600 & -3681 & -1628 \\
& $\mathrm{UCCSD}(\mathrm{T})$ & -8479 & -10137 & -7687 & -3703 & -1628 \\
\hline
\end{tabular}

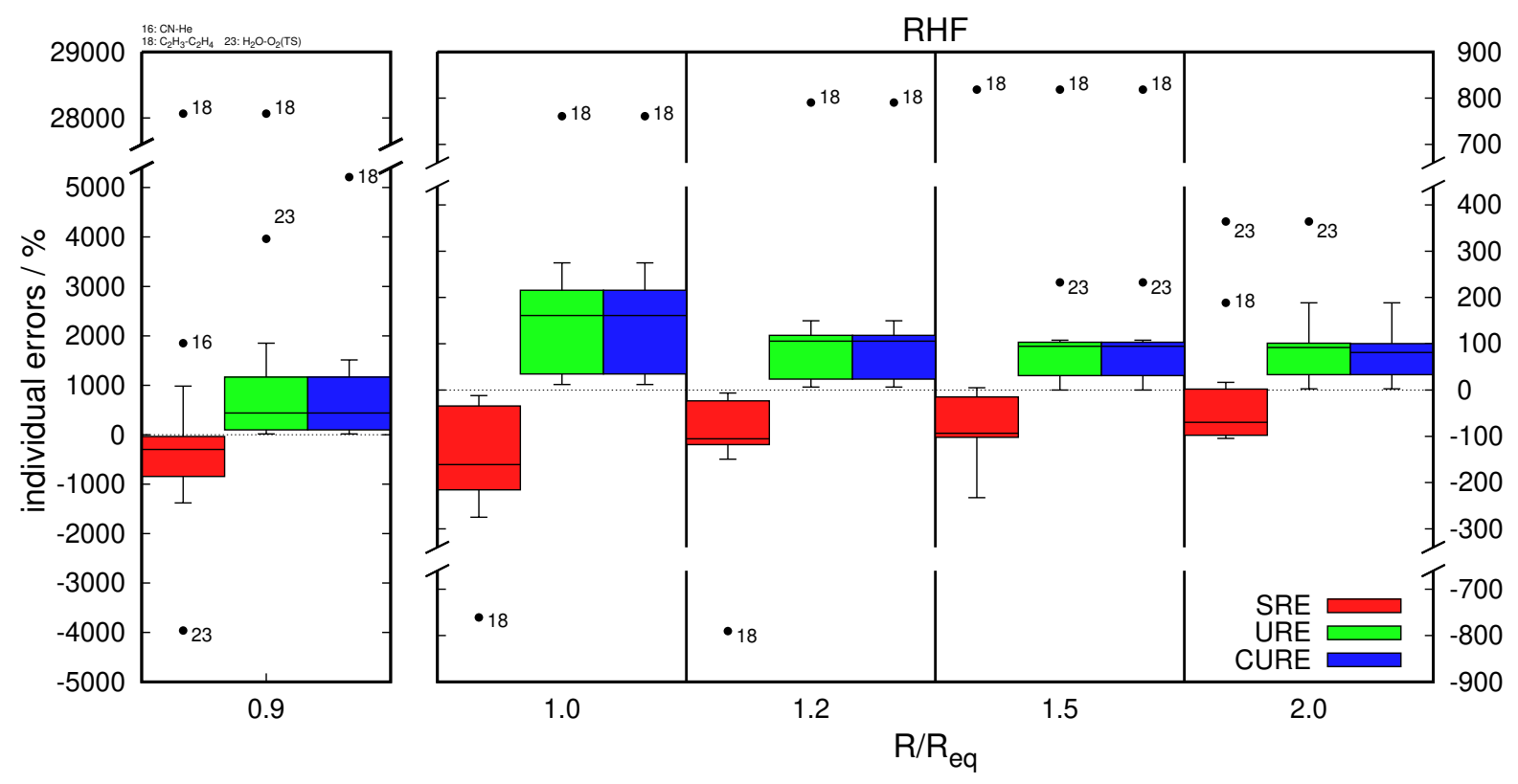

Figure S2: Box plot of signed relative error (SRE), unsigned relative error (URE) and capped unsigned relative error (CURE) (in percent) in the interaction energies at the restricted open-shell Hartree-Fock level of theory for the O24x5 data set. The box and outer fences encompass $50 \%$ and $95 \%$ of the distribution, respectively. Outliers are labeled according to the order of systems given in Tables S3-S12. 
Table S16: Relative difference of interaction energies obtained with $\operatorname{UCCSD}(\mathrm{T})$ with respect to $\operatorname{RCCSD}(\mathrm{T})$ (defined as $\left(E_{\mathrm{int}}^{\mathrm{UCCSD}(\mathrm{T})}-E_{\mathrm{int}}^{\mathrm{RCCSD}(\mathrm{T})}\right) / E_{\mathrm{int}}^{\mathrm{RCCSD}(\mathrm{T})} \times 100$ ). All energies were obtained with the aug-cc-pVTZ basis set.

\begin{tabular}{|c|c|c|c|c|c|}
\hline \multirow{2}{*}{ system } & \multicolumn{5}{|c|}{$R / R_{\mathrm{e}}$} \\
\hline & 0.9 & 1.0 & 1.2 & 1.5 & 2.0 \\
\hline \multicolumn{6}{|c|}{ dispersion } \\
\hline $\mathrm{CN}-\mathrm{He}$ & -2.92 & 1.10 & 0.88 & 0.29 & -3.45 \\
\hline $\mathrm{NH}-\mathrm{He}$ & 1.78 & 0.29 & 0.14 & -0.37 & -3.56 \\
\hline $\mathrm{C}_{2} \mathrm{H}_{3}-\mathrm{C}_{2} \mathrm{H}_{4}$ & -11.28 & 1.10 & 0.97 & -0.82 & -2.62 \\
\hline $\mathrm{O}_{2}-\mathrm{H}_{2}$ & 17.28 & 1.17 & 0.53 & 0.45 & 0.37 \\
\hline $\mathrm{NH}-\mathrm{Ar}$ & 0.85 & 0.31 & 0.30 & 0.53 & 0.35 \\
\hline $\mathrm{CN}-\mathrm{Ar}$ & -5.30 & 0.97 & 0.15 & 0.03 & -0.65 \\
\hline $\mathrm{O}_{2}-\mathrm{N}_{2}$ & 10.75 & 0.93 & 0.56 & 0.12 & 0.14 \\
\hline $\mathrm{O}_{2}-\mathrm{O}_{2}$ & 2.48 & 0.76 & 0.73 & 0.75 & 0.38 \\
\hline \multicolumn{6}{|c|}{ electrostatic } \\
\hline $\mathrm{NH}-\mathrm{NH}$ & 0.38 & 0.17 & 0.13 & 0.08 & 0.03 \\
\hline $\mathrm{CH}_{2} \mathrm{O}-\mathrm{NH}_{2}$ & 0.56 & 0.19 & 0.07 & 0.03 & 0 \\
\hline $\mathrm{H}_{2} \mathrm{O}-\mathrm{Na}$ & 0.46 & 0.11 & 0.07 & 0.04 & 0.01 \\
\hline $\mathrm{H}_{2} \mathrm{O}-\mathrm{OH}$ & 0.13 & 0.04 & 0.01 & 0 & -0.01 \\
\hline $\mathrm{H}_{2} \mathrm{O}-\mathrm{O}_{2} \mathrm{H}$ & 0.74 & 0.40 & 0.30 & 0.31 & 0.35 \\
\hline $\mathrm{Li}-\mathrm{NH}_{3}(\mathrm{gm})$ & 0.09 & 0.06 & 0.07 & 0.08 & 0.05 \\
\hline \multicolumn{6}{|c|}{ mixed } \\
\hline $\mathrm{Li}-\mathrm{O}_{2}$ & 3.04 & 0.66 & 0.41 & 0.39 & 0.38 \\
\hline $\mathrm{CN}-\mathrm{H}_{2}$ & 7.08 & 0.86 & 0.41 & -0.18 & -4.30 \\
\hline $\mathrm{Li}-\mathrm{NH}_{3}(\mathrm{~lm})$ & 1.21 & 0.45 & 0.10 & 0.03 & 0 \\
\hline $\mathrm{Na}-\mathrm{Li}$ & 0 & 0 & 0 & 0 & 0 \\
\hline $\mathrm{CO}_{2}-\mathrm{O}_{2}$ & 1.13 & 0.38 & 0.24 & 0.05 & 0.16 \\
\hline $\mathrm{C}_{2} \mathrm{H}_{3}-\mathrm{CO}_{2}$ & 1.36 & 0.32 & 0.14 & 0.07 & -0.02 \\
\hline $\mathrm{He}_{2}^{*}$ & 0 & 0 & 0 & 0 & 0 \\
\hline $\mathrm{HF}-\mathrm{CO}^{+}$ & 0.38 & 0.58 & 1.13 & 0.60 & 0 \\
\hline
\end{tabular}


Table S17: Interaction energies for the $\mathrm{He}^{*}-\mathrm{He}^{*}$ and $\mathrm{Na}-\mathrm{Li}$ dimers at the MP2 and MP2c levels of theory. $E_{\mathrm{DISP}, \text { unc }}^{(2)}$ and $E_{\mathrm{DISP}, \text { cpld }}^{(2)}$ denote the sum of second-order dispersion and exchange-dispersion interaction energy contributions calculated at the SAPT(UHF) level of theory in the uncoupled and coupled approximations, respectively. MP2c denotes the MP2 interaction energy corrected for the coupled dispersion. Both MP2 and RCCSD(T) results are extrapolated to the CBS limit, the latter being the benchmark. SAPT(UHF) calculations were performed in the aug-cc-pVQZ and d-aug-cc-pVQZ basis sets for $\mathrm{Na}-\mathrm{Li}$ and $\mathrm{He}^{*}-\mathrm{He}^{*}$, respectively. All energies in $\mathrm{cm}^{-1}$.

\begin{tabular}{lrrrrr}
\hline \multicolumn{5}{c}{$\mathrm{He}\left({ }^{3} \mathrm{~S}\right)-\mathrm{He}\left({ }^{3} \mathrm{~S}\right)$} \\
$R / R_{\text {eq }}$ & $E_{\text {DISP,unc }}^{(2)}$ & $E_{\text {DISP,cpld }}^{(2)}$ & $\mathrm{MP} 2$ & $\mathrm{MP} 2 \mathrm{c}$ & $\mathrm{RCCSD}(\mathrm{T})$ \\
\hline 0.9 & -744.0 & -2331 & -135.6 & -1722 & -826.9 \\
1.0 & -537.7 & -1771 & -373.8 & -1609 & -1019 \\
1.2 & -287.5 & -996.4 & -290.3 & -1001 & -790.2 \\
1.5 & -109.7 & -381.9 & -106.1 & -379.0 & -341.3 \\
2.0 & -21.95 & -76.82 & -20.75 & -75.85 & -74.18 \\
& & \multicolumn{5}{c}{$\mathrm{Na}-\mathrm{Li}$} \\
\hline 0.9 & -335.4 & -848.9 & 113.5 & -400.2 & -210.4 \\
1.0 & -226.7 & -587.5 & -5.76 & -366.5 & -259.3 \\
1.2 & -104.9 & -276.3 & -50.70 & -222.1 & -188.2 \\
1.5 & -32.26 & -86.91 & -26.98 & -81.31 & -75.17 \\
2.0 & -5.27 & -14.70 & -5.18 & -14.61 & -14.25 \\
\hline
\end{tabular}



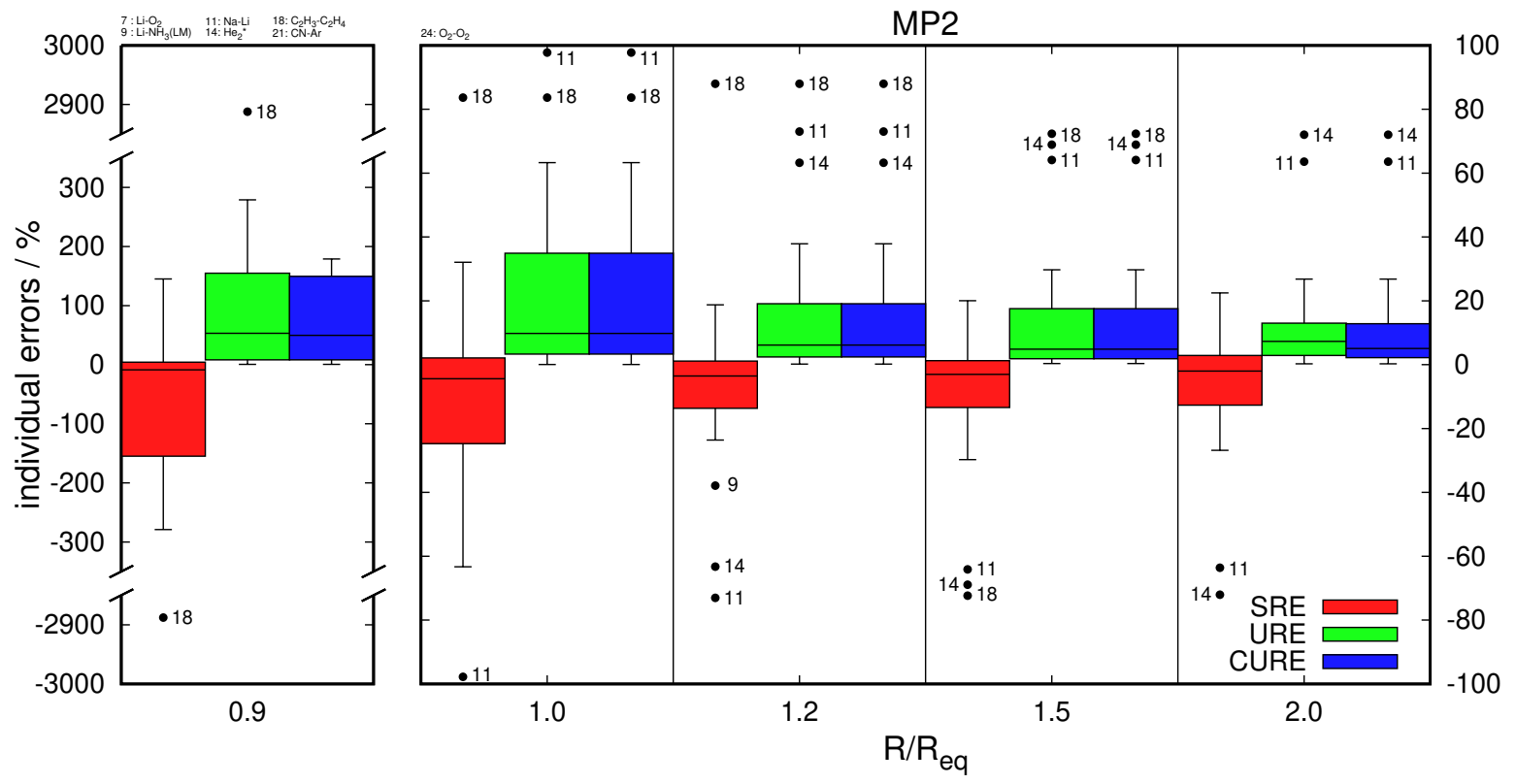

Figure S3: Box plot of signed relative error (SRE), unsigned relative error (URE) and capped unsigned relative error (CURE) (in percent) in the interaction energies at the restricted openshell MP2 level of theory for the O24x5 data set. The box and outer fences encompass $50 \%$ and $95 \%$ of the distribution, respectively. Outliers are labeled according to the order of systems given in Tables S3-S12.
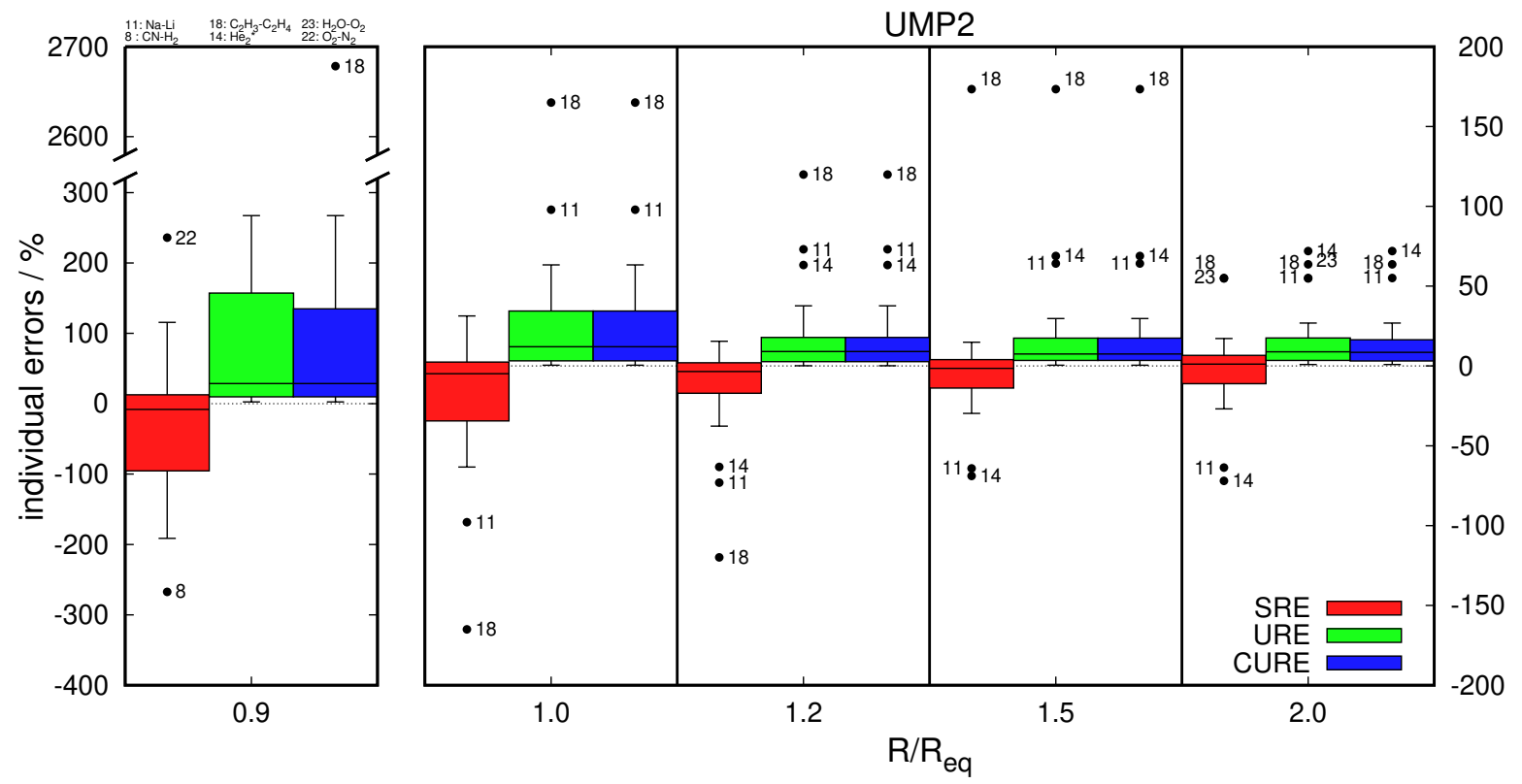

Figure S4: Box plot of signed relative error (SRE), unsigned relative error (URE) and capped unsigned relative error (CURE) (in percent) in the interaction energies at the unrestricted MP2 level of theory for the $\mathrm{O} 24 \times 5$ data set. The box and outer fences encompass $50 \%$ and $95 \%$ of the distribution, respectively. Outliers are labeled according to the order of systems given in Tables S3-S12. 

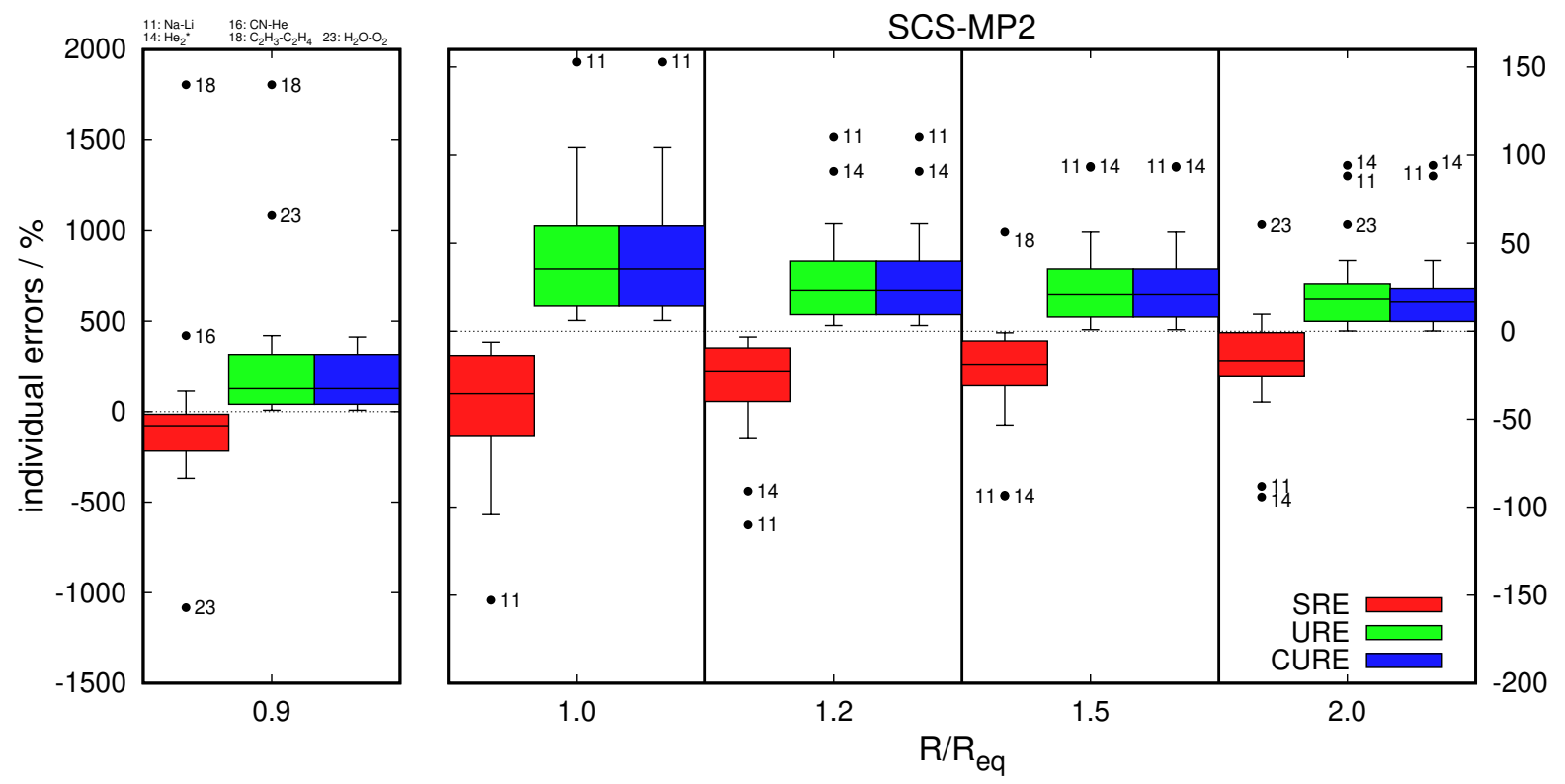

Figure S5: Box plot of signed relative error (SRE), unsigned relative error (URE) and capped unsigned relative error (CURE) (in percent) in the interaction energies at the SCS-MP2 level of theory for the O24x5 data set. The box and outer fences encompass $50 \%$ and $95 \%$ of the distribution, respectively. Outliers are labeled according to the order of systems given in Tables S3-S12.
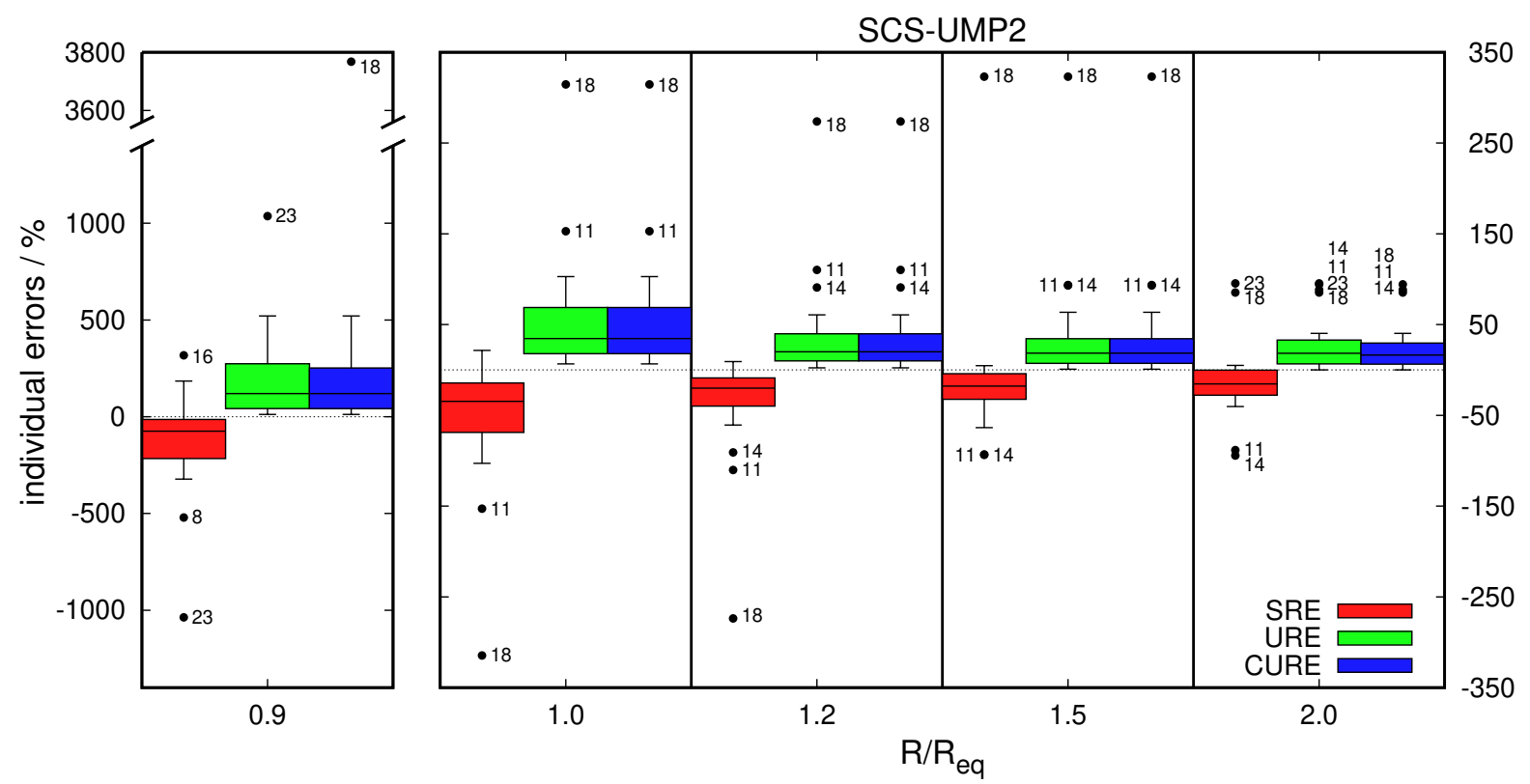

Figure S6: Box plot of signed relative error (SRE), unsigned relative error (URE) and capped unsigned relative error (CURE) (in percent) in the interaction energies at the SCS-UMP2 level of theory for the $\mathrm{O} 24 \mathrm{x} 5$ data set. The box and outer fences encompass $50 \%$ and $95 \%$ of the distribution, respectively. Outliers are labeled according to the order of systems given in Tables S3-S12. 


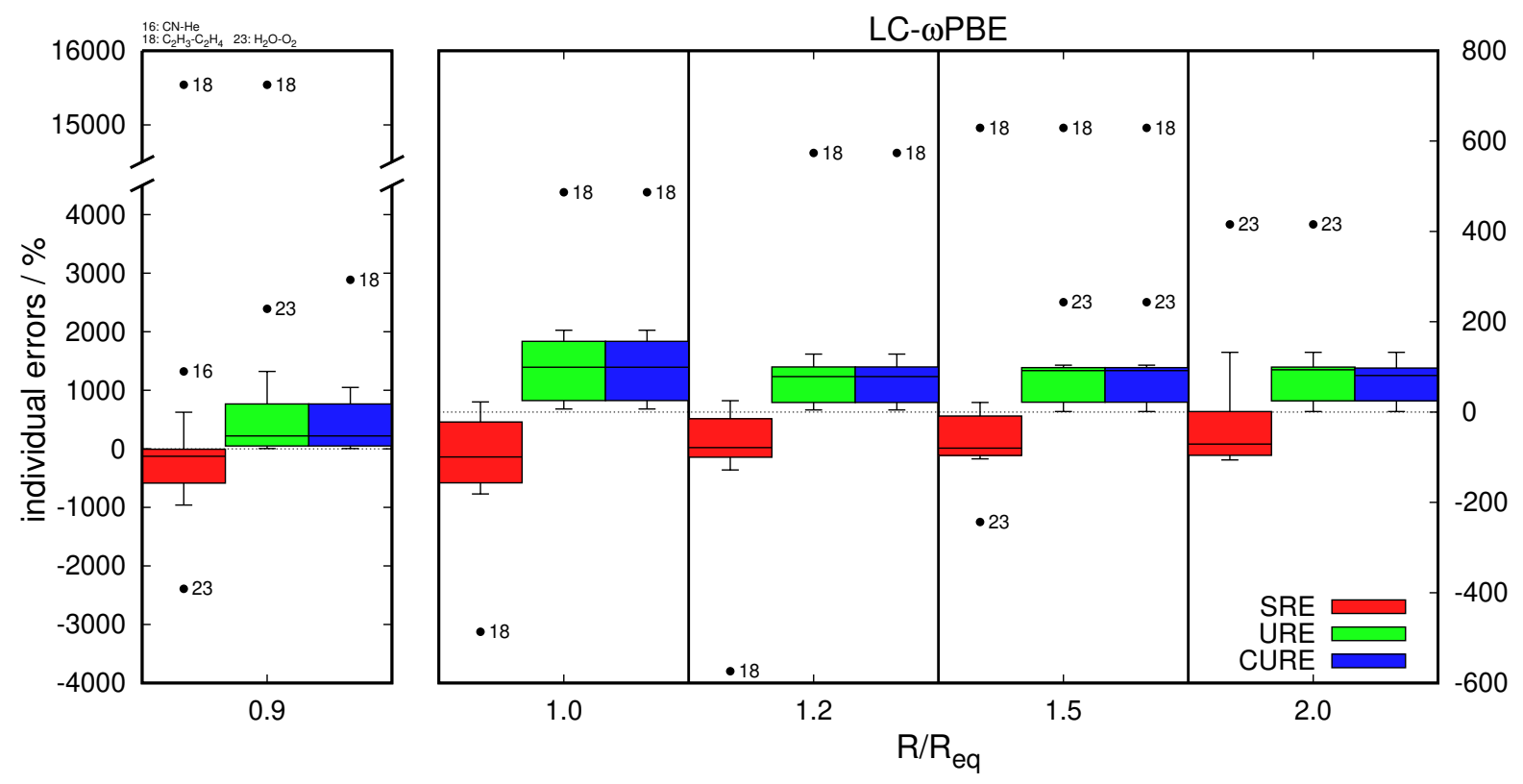

Figure S7: Box plot of signed relative error (SRE), unsigned relative error (URE) and capped unsigned relative error (CURE) (in percent) in the interaction energies at the LC- $\omega \mathrm{PBE}$ level of theory for the O24x5 data set. The box and outer fences encompass $50 \%$ and $95 \%$ of the distribution, respectively. Outliers are labeled according to the order of systems given in Tables S3-S12.

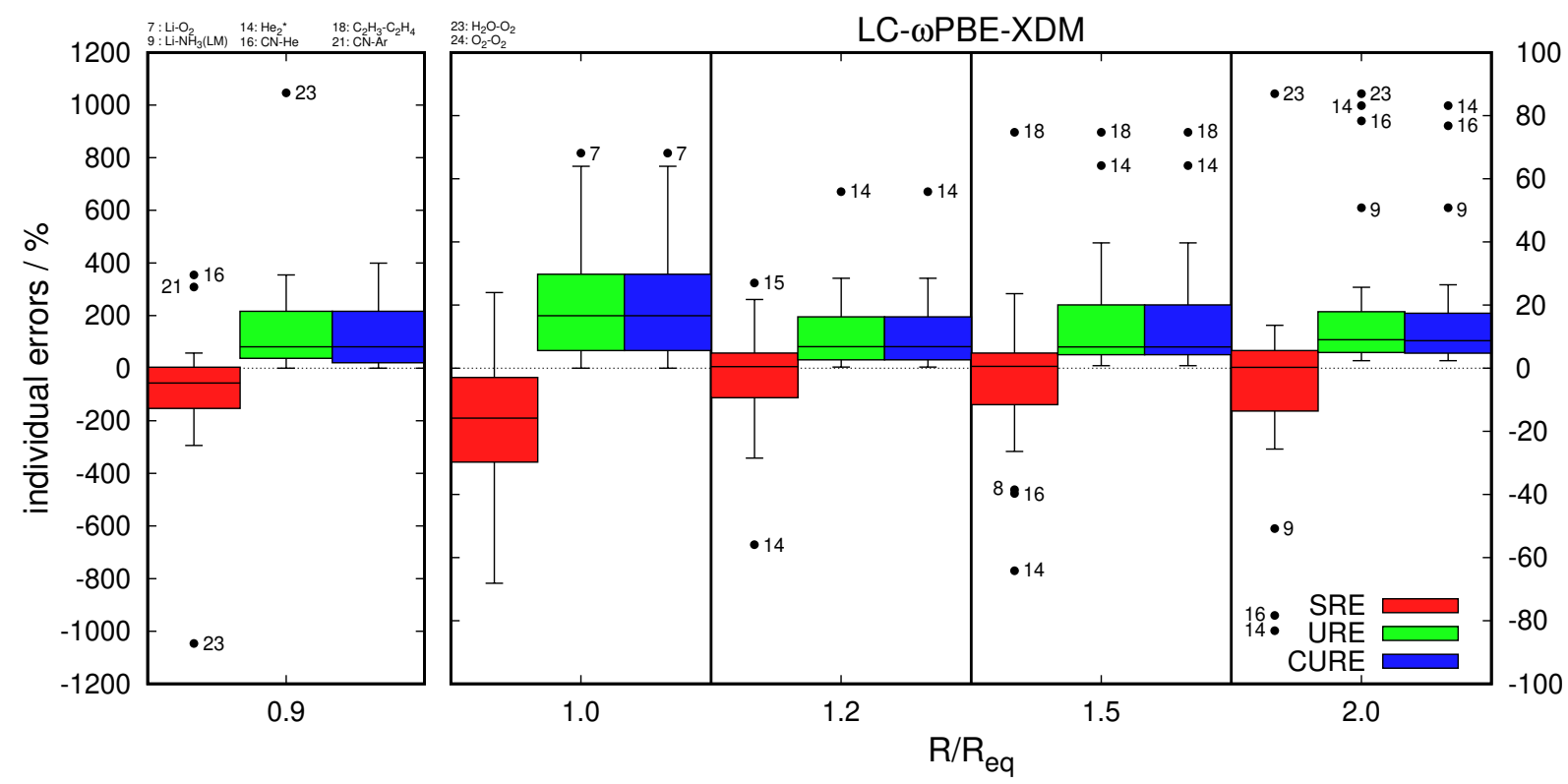

Figure S8: Box plot of signed relative error (SRE), unsigned relative error (URE) and capped unsigned relative error (CURE) (in percent) in the interaction energies at the LC- $\omega$ PBE level of theory with the XDM dispersion correction for the $\mathrm{O} 24 \mathrm{x} 5$ data set. The box and outer fences encompass $50 \%$ and $95 \%$ of the distribution, respectively. Outliers are labeled according to the order of systems given in Tables S3-S12. 


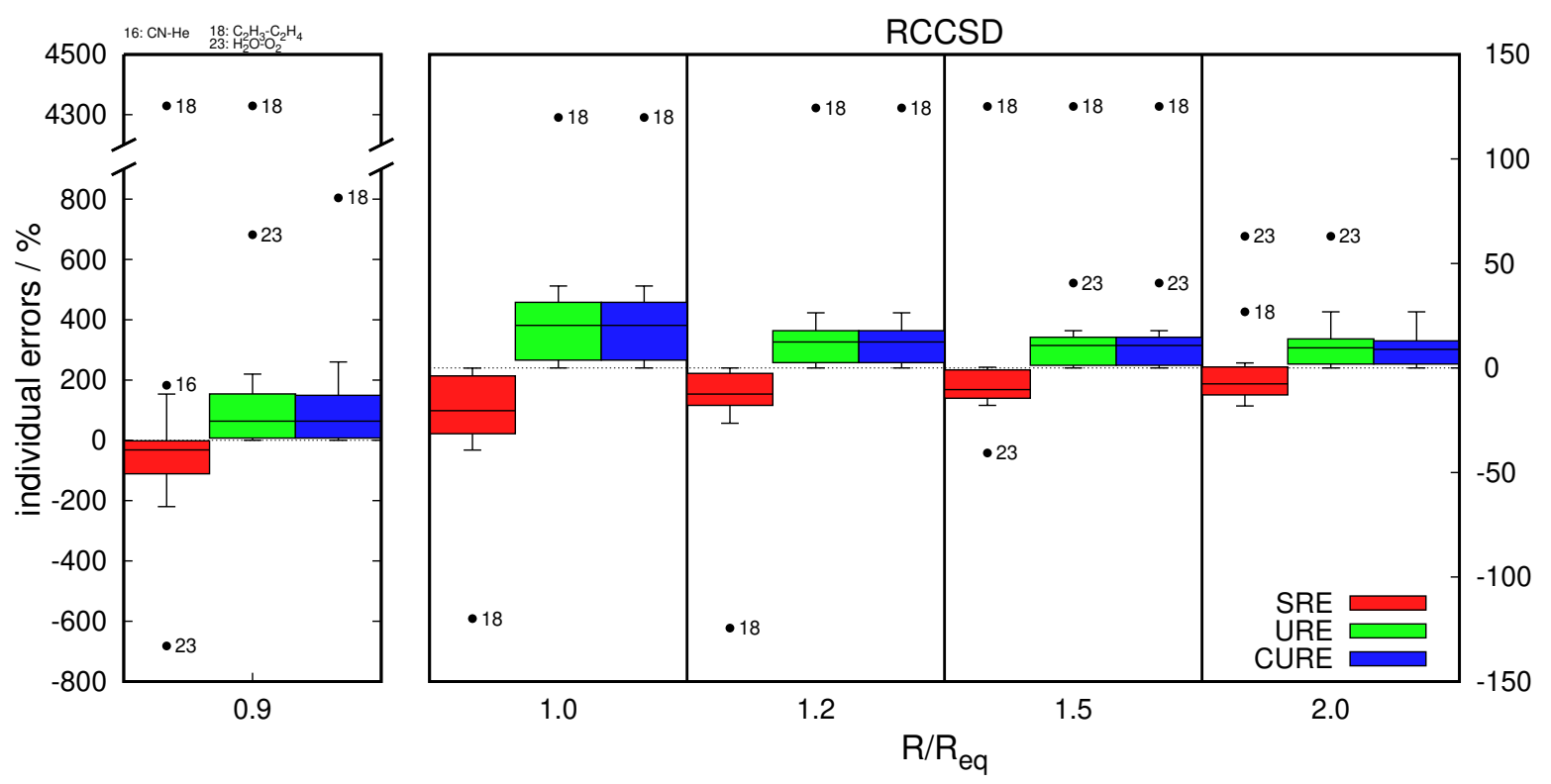

Figure S9: Box plot of signed relative error (SRE), unsigned relative error (URE) and capped unsigned relative error (CURE) (in percent) in the interaction energies at the RCCSD level of theory for the O24x5 data set. The box and outer fences encompass $50 \%$ and $95 \%$ of the distribution, respectively. Outliers are labeled according to the order of systems given in Tables S3-S12.

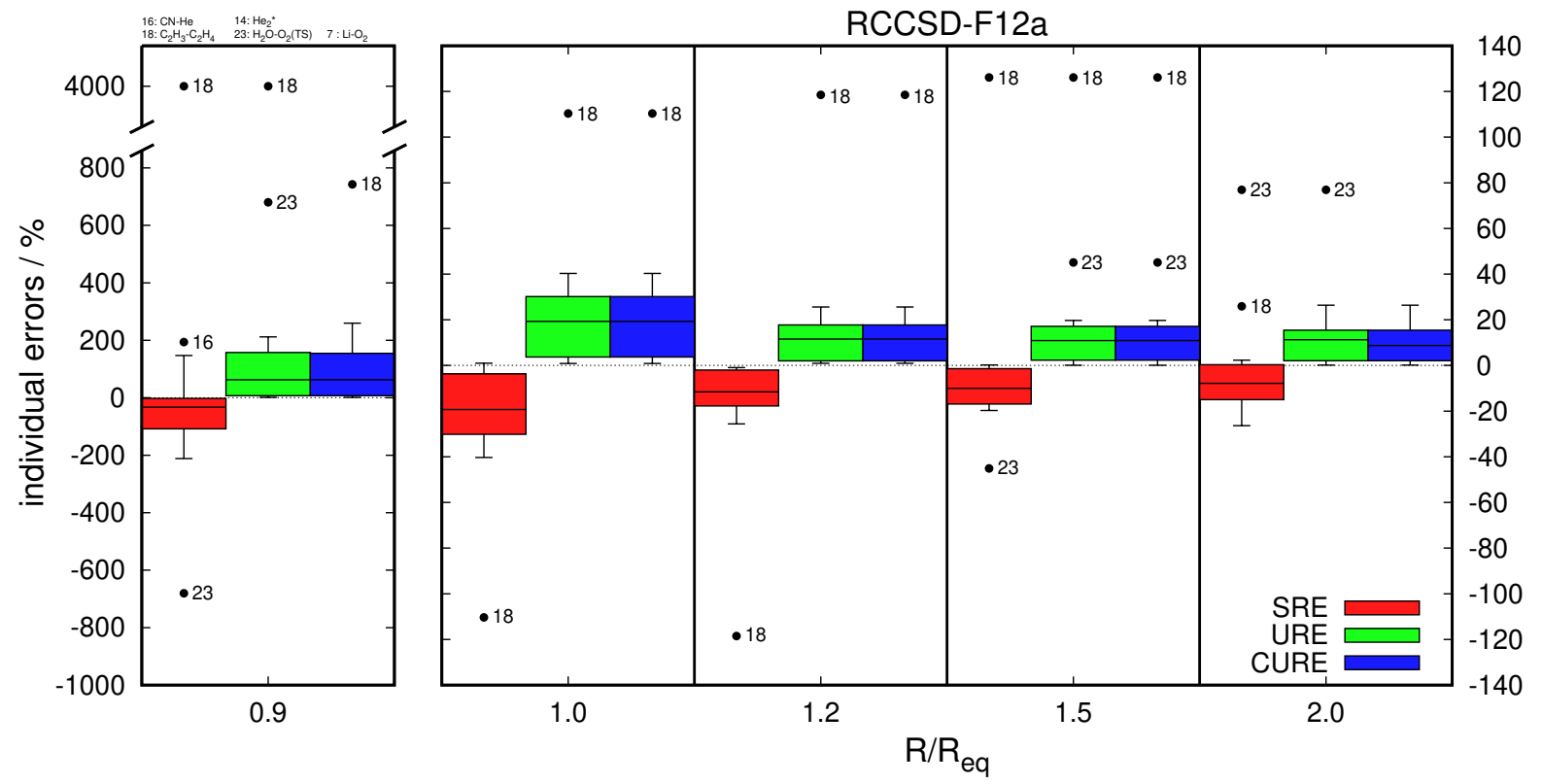

Figure S10: Box plot of signed relative error (SRE), unsigned relative error (URE) and capped unsigned relative error (CURE) (in percent) in the interaction energies at the RCCSD-F12a level of theory for the O24x5 data set. The box and outer fences encompass $50 \%$ and $95 \%$ of the distribution, respectively. Outliers are labeled according to the order of systems given in Tables S3-S12. 


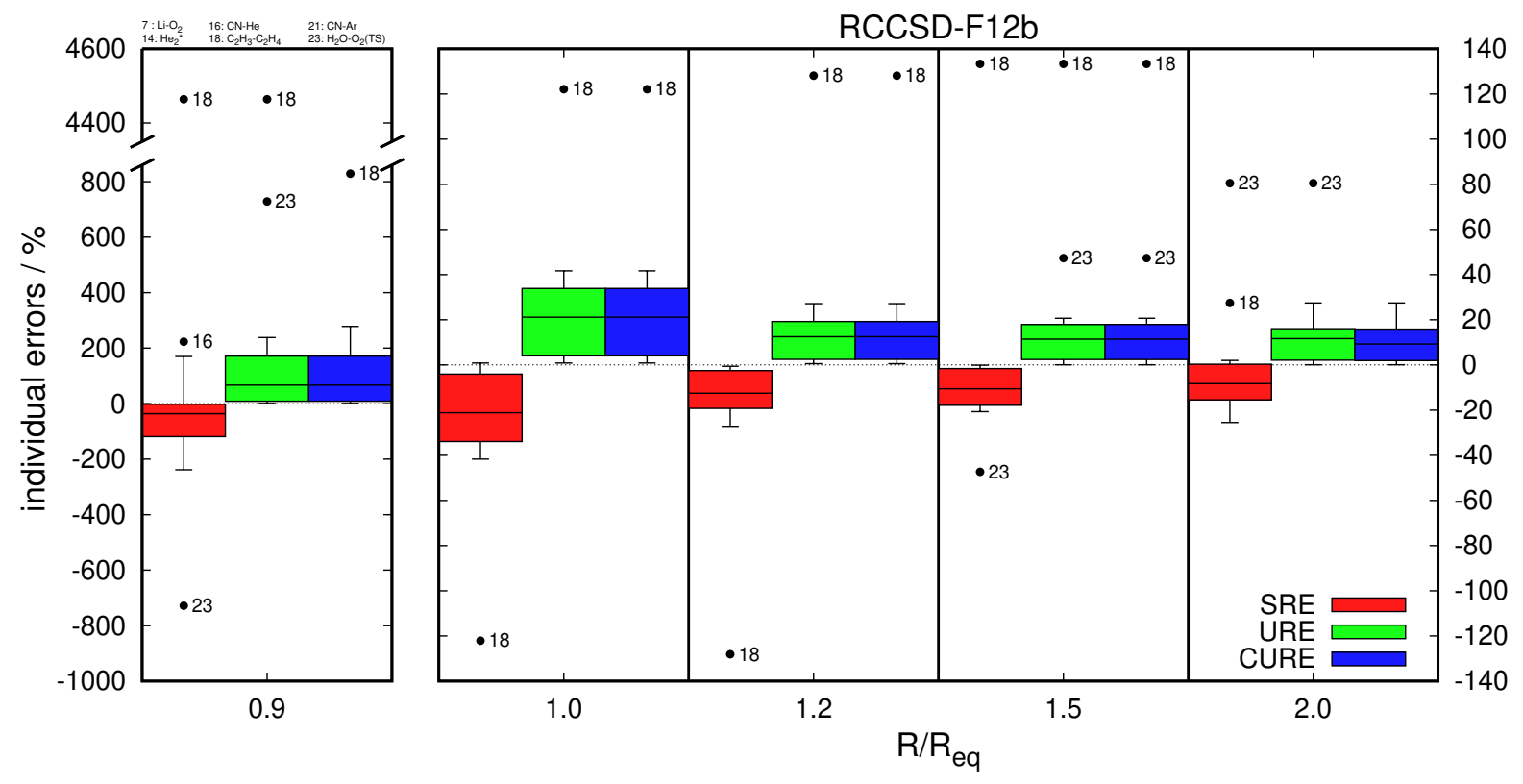

Figure S11: Box plot of signed relative error (SRE), unsigned relative error (URE) and capped unsigned relative error (CURE) (in percent) in the interaction energies at the RCCSD-F12b level of theory for the O24x 5 data set. The box and outer fences encompass $50 \%$ and $95 \%$ of the distribution, respectively. Outliers are labeled according to the order of systems given in Tables S3-S12.

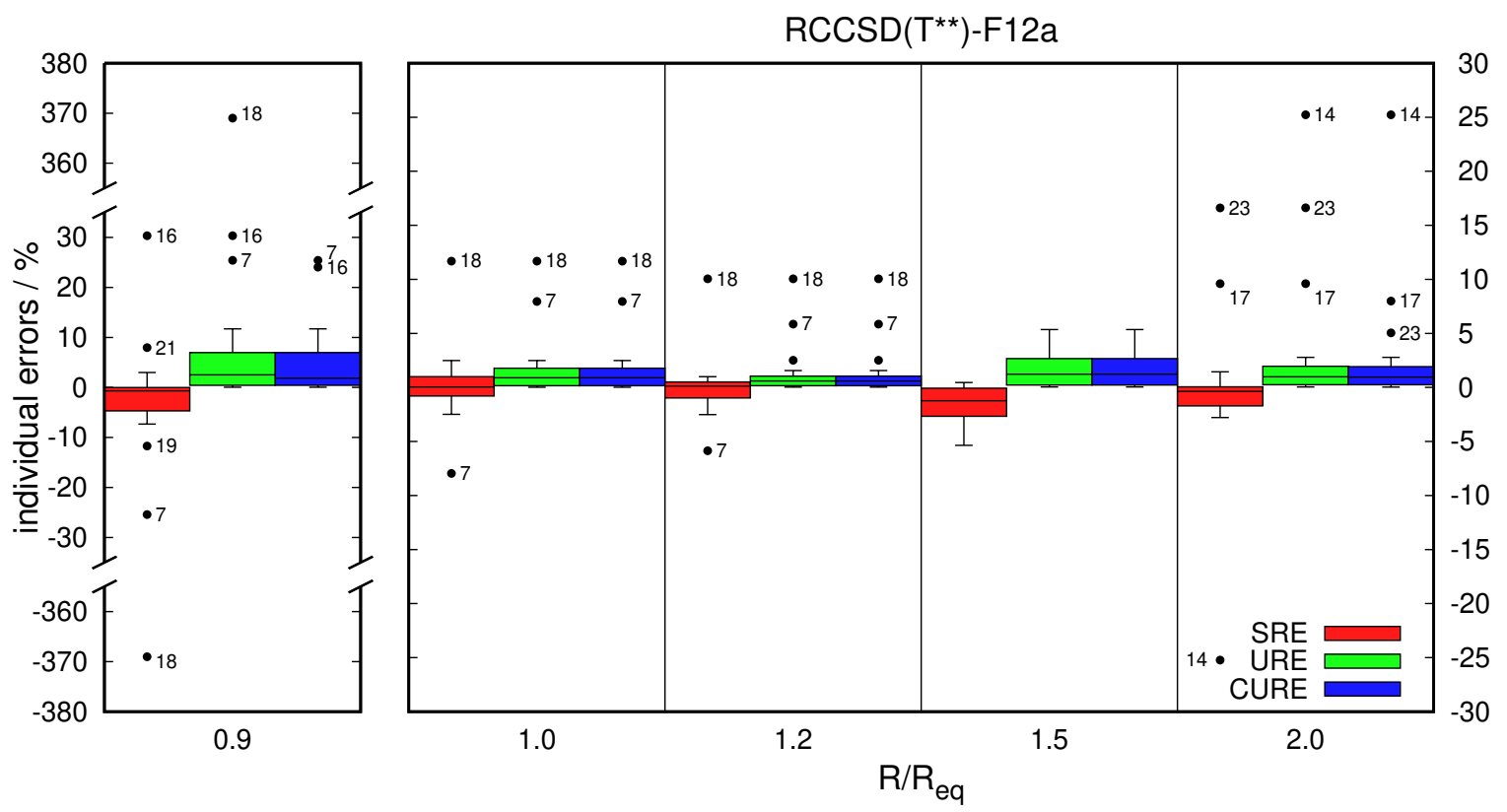

Figure S12: Box plot of signed relative error (SRE), unsigned relative error (URE) and capped unsigned relative error (CURE) (in percent) in the interaction energies at the $\operatorname{RCCSD}\left(\mathrm{T}^{* *}\right)$-F12a level of theory for the $\mathrm{O} 24 \times 5$ data set. The box and outer fences encompass $50 \%$ and $95 \%$ of the distribution, respectively. Outliers are labeled according to the order of systems given in Tables S3-S12. 

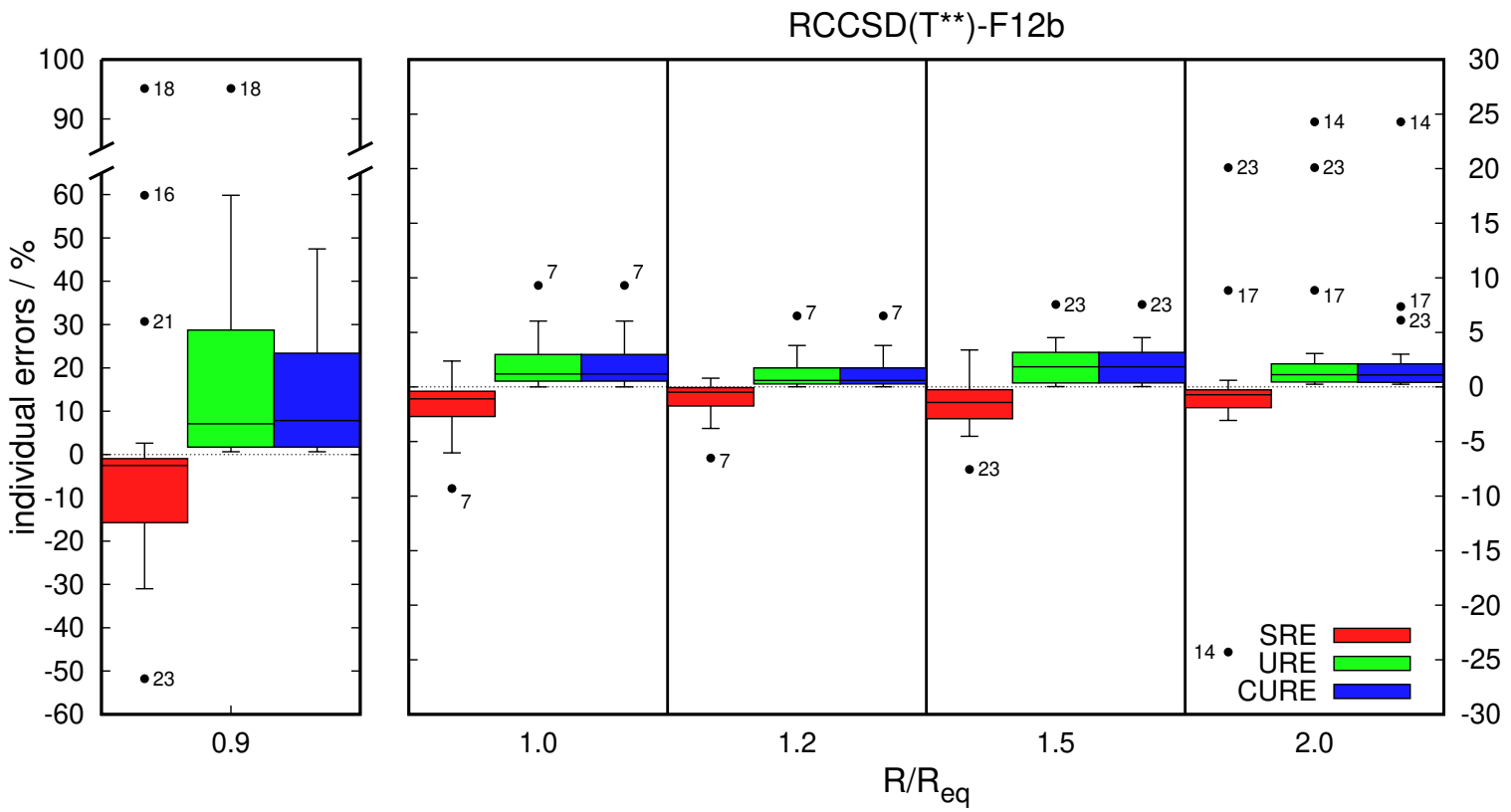

Figure S13: Box plot of signed relative error (SRE), unsigned relative error (URE) and capped unsigned relative error (CURE) (in percent) in the interaction energies at the $\operatorname{RCCSD}\left(\mathrm{T}^{* *}\right)$-F12b level of theory for the $\mathrm{O} 24 \mathrm{x} 5$ data set. The box and outer fences encompass $50 \%$ and $95 \%$ of the distribution, respectively. Outliers are labeled according to the order of systems given in Tables S3-S12.
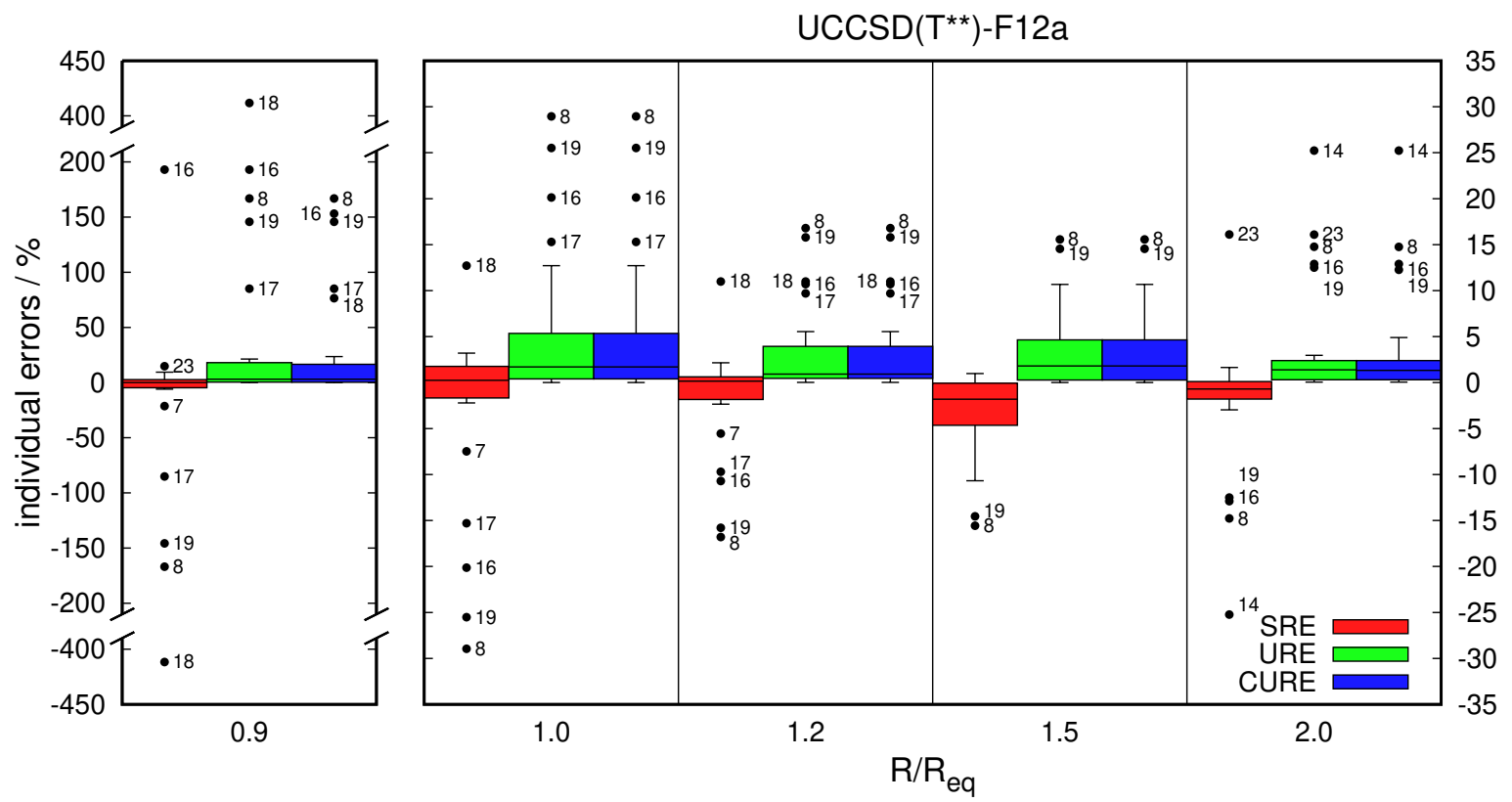

Figure S14: Box plot of signed relative error (SRE), unsigned relative error (URE) and capped unsigned relative error (CURE) (in percent) in the interaction energies at the $\mathrm{UCCSD}\left(\mathrm{T}^{* *}\right)-\mathrm{F} 12 \mathrm{a}$ level of theory for the $\mathrm{O} 24 \mathrm{x} 5$ data set. The box and outer fences encompass $50 \%$ and $95 \%$ of the distribution, respectively. Outliers are labeled according to the order of systems given in Tables S3-S12. 

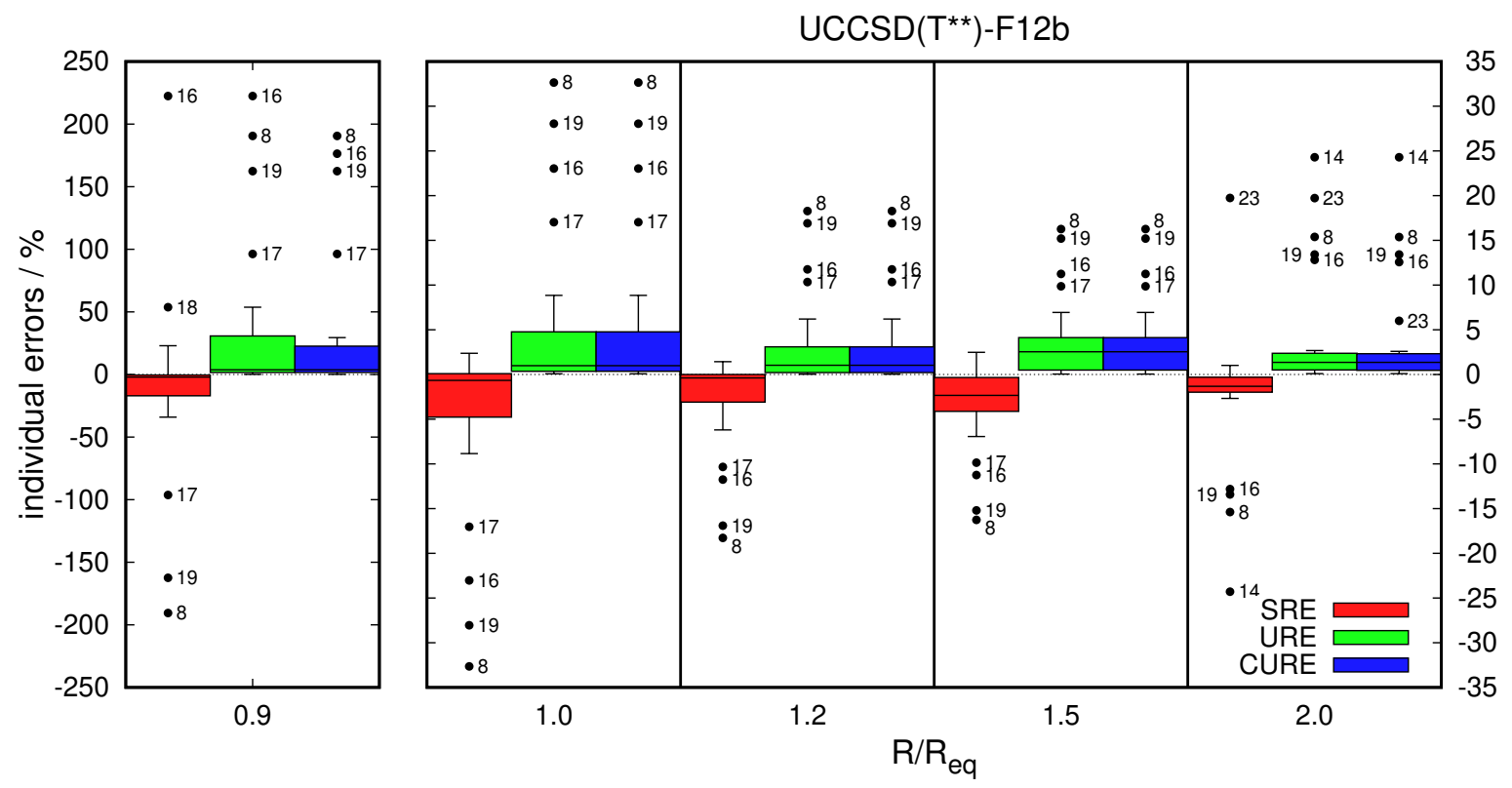

Figure S15: Box plot of signed relative error (SRE), unsigned relative error (URE) and capped unsigned relative error (CURE) (in percent) in the interaction energies at the $\mathrm{UCCSD}\left(\mathrm{T}^{* *}\right)$-F12b level of theory for the O24x5 data set. The box and outer fences encompass $50 \%$ and $95 \%$ of the distribution, respectively. Outliers are labeled according to the order of systems given in Tables S3-S12.

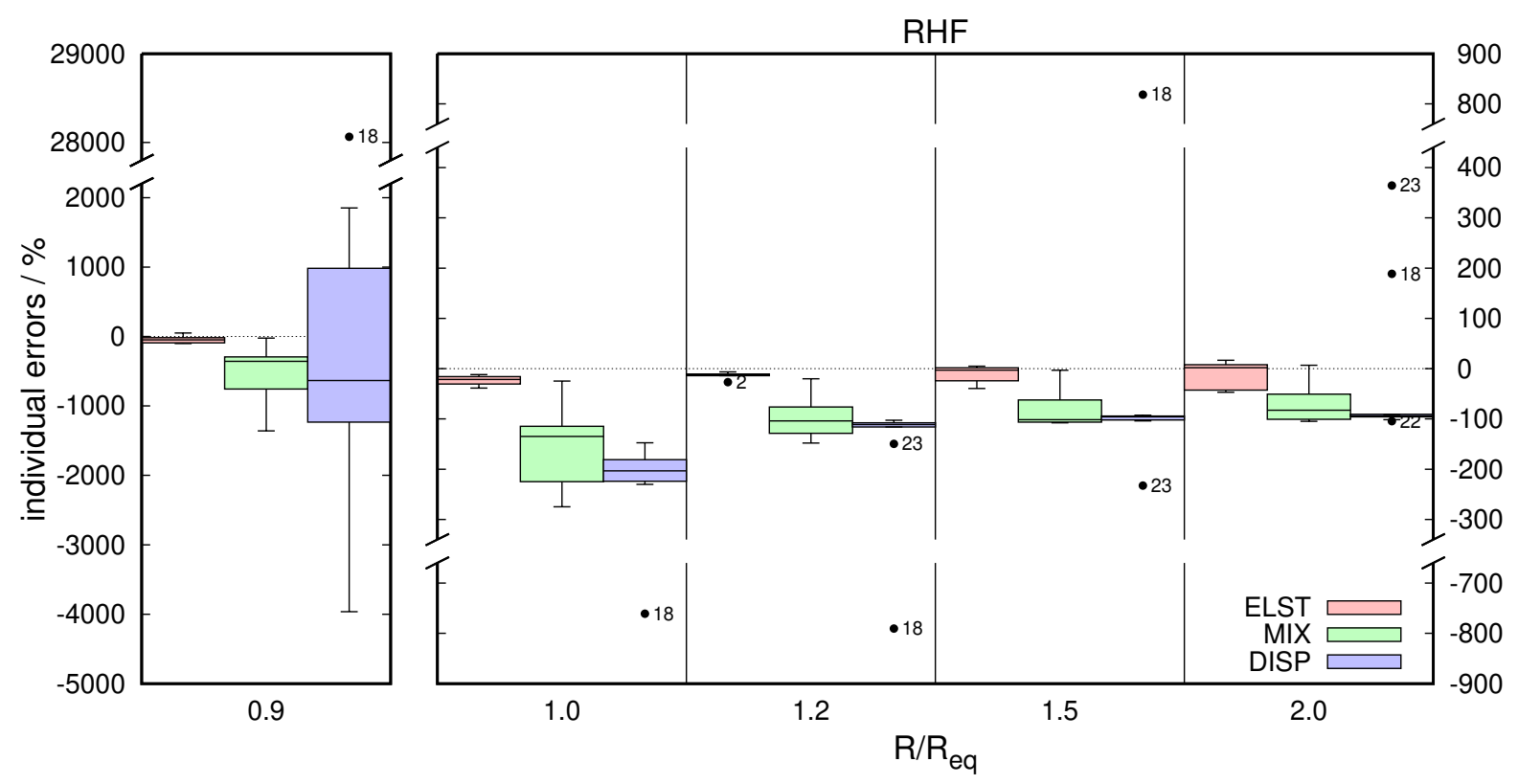

Figure S16: Box plot of signed relative errors (SRE, in percent) in the interaction energies at the ROHF level of theory for the O24x5 data set divided in the electrostatic (ELST), mixed (MIX) and dispersion (DISP) subsets. The box and outer fences encompass 50\% and 95\% of the distribution, respectively. Outliers are labeled according to the order of systems given in Tables S3-S12. 

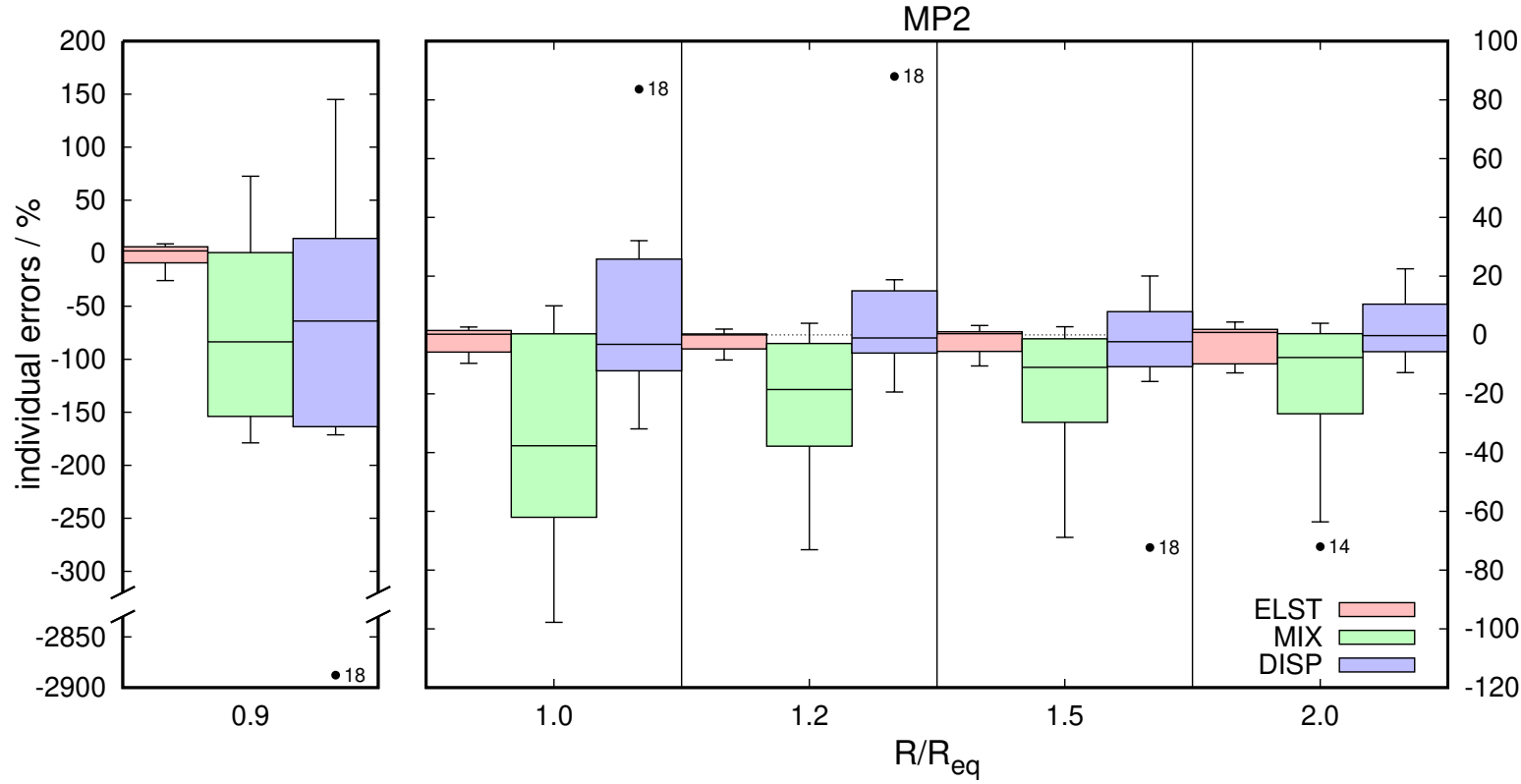

Figure S17: Box plot of signed relative errors (SRE, in percent) in the interaction energies at the MP2 level of theory for the O24x5 data set divided in the electrostatic (ELST), mixed (MIX) and dispersion (DISP) subsets. The box and outer fences encompass 50\% and $95 \%$ of the distribution, respectively. Outliers are labeled according to the order of systems given in Tables S3-S12.
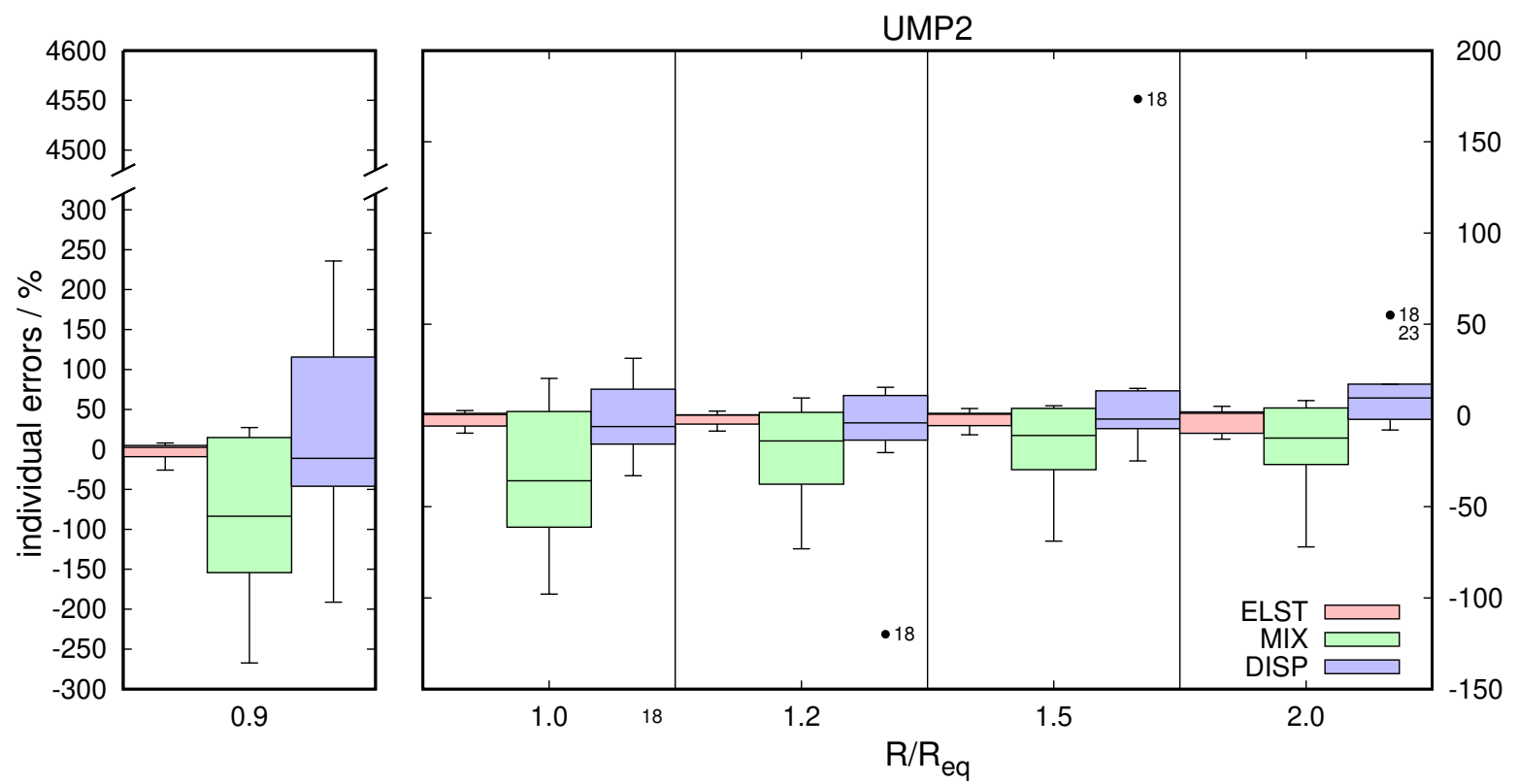

Figure S18: Box plot of signed relative errors (SRE, in percent) in the interaction energies at the UMP2 level of theory for the O24x5 data set divided in the electrostatic (ELST), mixed (MIX) and dispersion (DISP) subsets. The box and outer fences encompass 50\% and $95 \%$ of the distribution, respectively. Outliers are labeled according to the order of systems given in Tables S3-S12. 


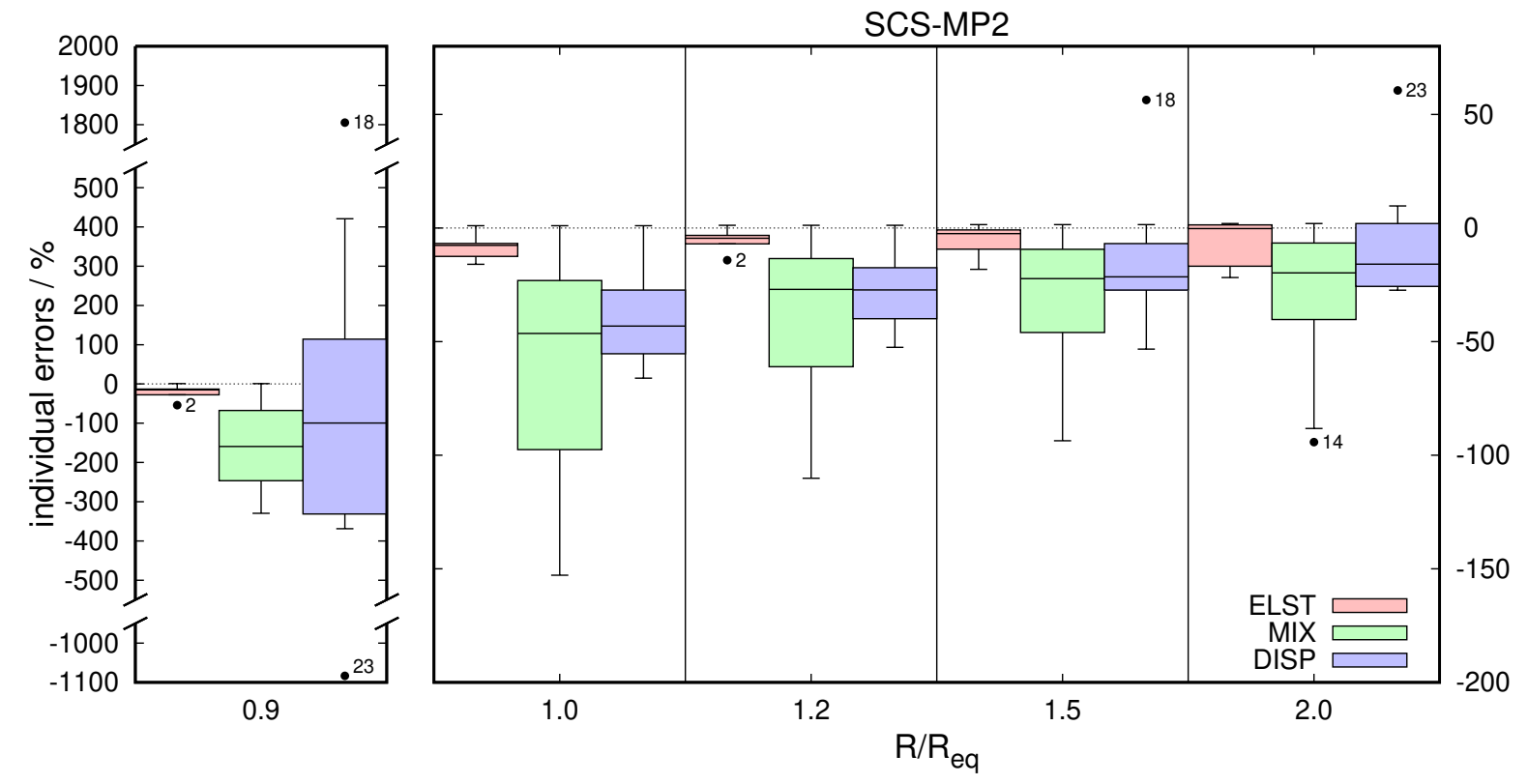

Figure S19: Box plot of signed relative errors (SRE, in percent) in the interaction energies at the SCS-MP2 level of theory for the O24x5 data set divided in the electrostatic (ELST), mixed (MIX) and dispersion (DISP) subsets. The box and outer fences encompass $50 \%$ and $95 \%$ of the distribution, respectively. Outliers are labeled according to the order of systems given in Tables S3-S12.
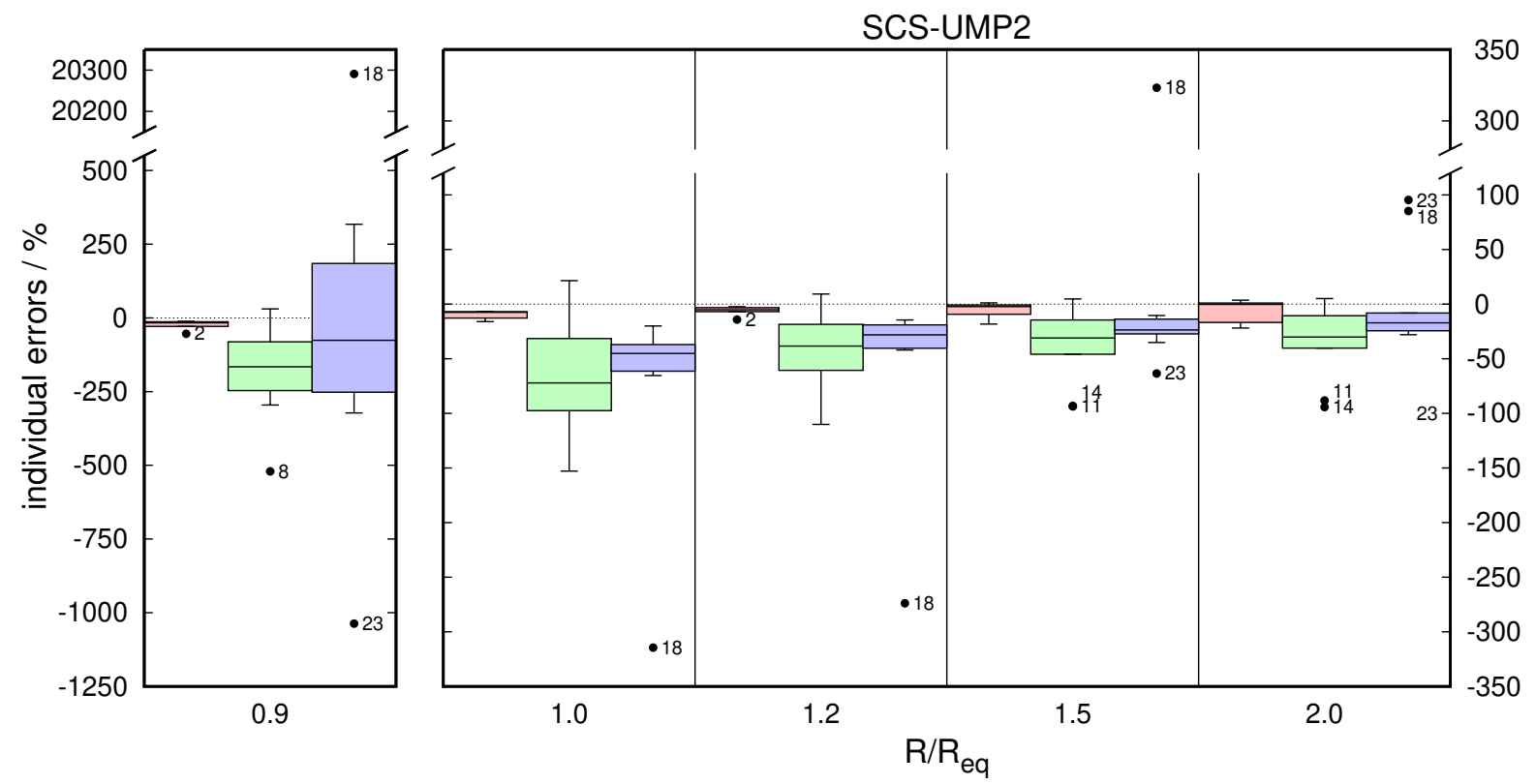

Figure S20: Box plot of signed relative errors (SRE, in percent) in the interaction energies at the SCS-UMP2 level of theory for the O24x5 data set divided in the electrostatic (ELST), mixed (MIX) and dispersion (DISP) subsets. The box and outer fences encompass $50 \%$ and $95 \%$ of the distribution, respectively. Outliers are labeled according to the order of systems given in Tables S3-S12. 

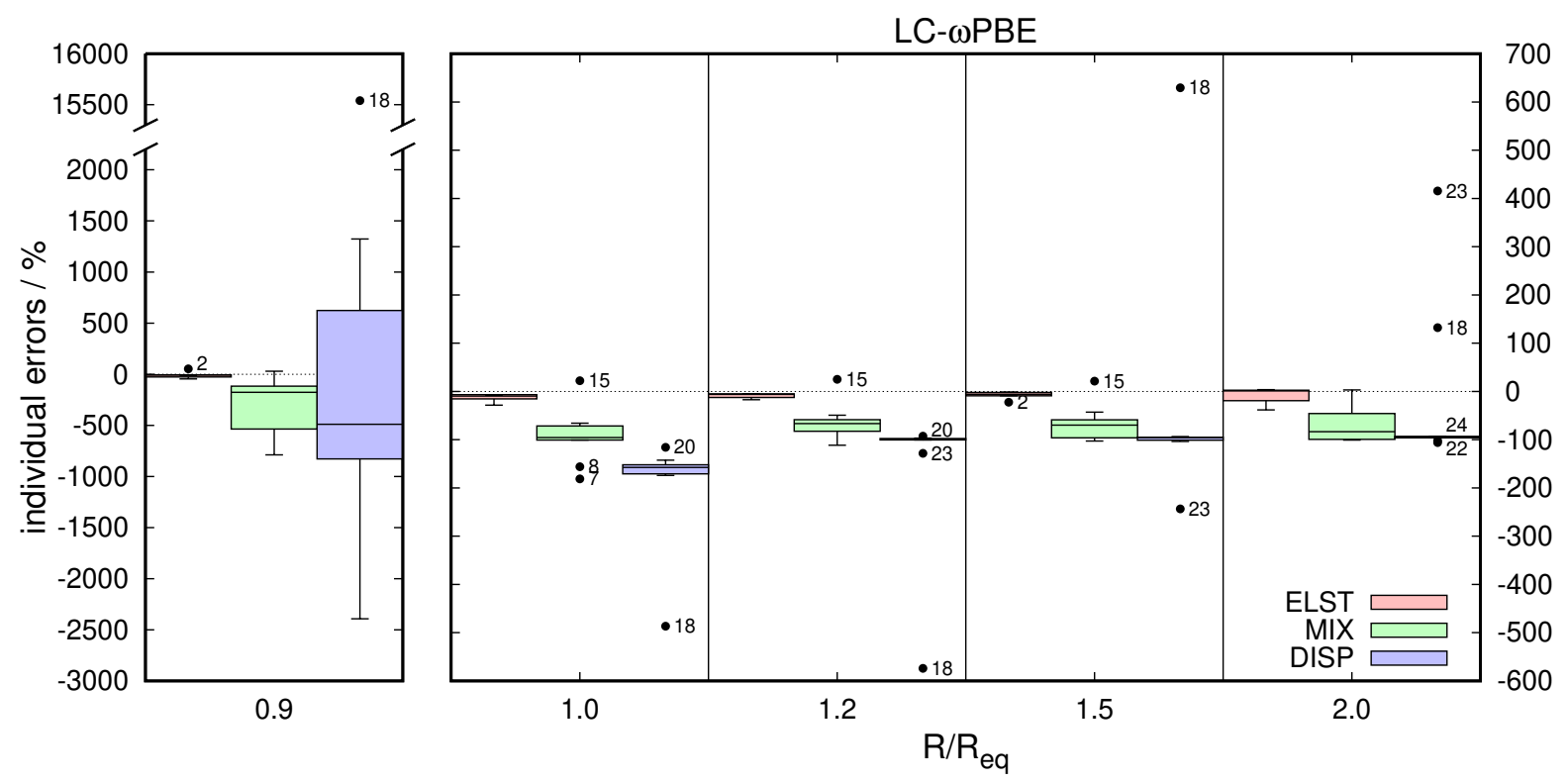

Figure S21: Box plot of signed relative errors (SRE, in percent) in the interaction energies at the LC- $\omega \mathrm{PBE}$ level of theory for the $\mathrm{O} 24 \mathrm{x} 5$ data set divided in the electrostatic (ELST), mixed (MIX) and dispersion (DISP) subsets. The box and outer fences encompass $50 \%$ and $95 \%$ of the distribution, respectively. Outliers are labeled according to the order of systems given in Tables S3-S12.

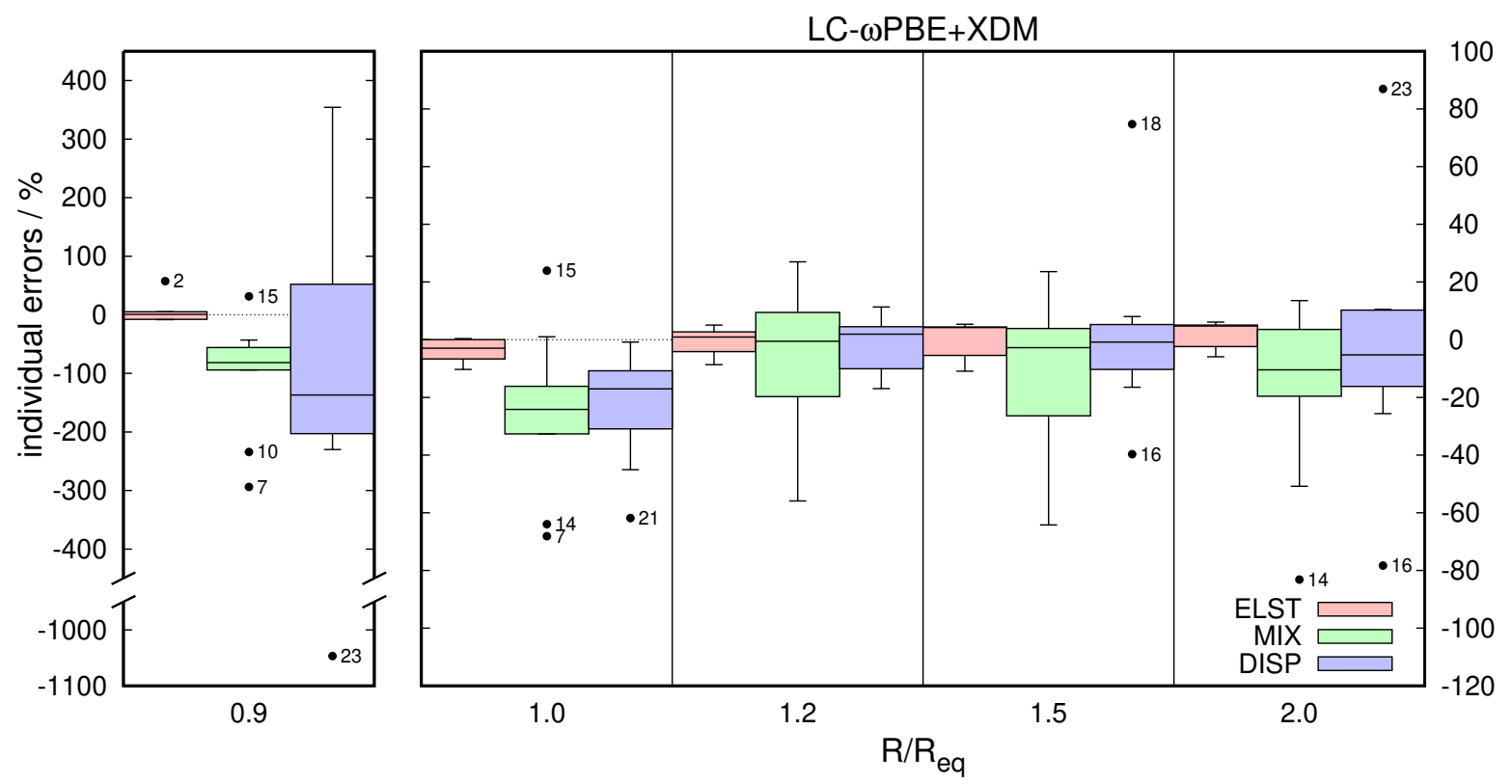

Figure S22: Box plot of signed relative errors (SRE, in percent) in the interaction energies at the $\mathrm{LC}-\omega \mathrm{PBE}+\mathrm{XDM}$ level of theory for the $\mathrm{O} 24 \times 5$ data set divided in the electrostatic (ELST), mixed (MIX) and dispersion (DISP) subsets. The box and outer fences encompass $50 \%$ and $95 \%$ of the distribution, respectively. Outliers are labeled according to the order of systems given in Tables S3-S12. 


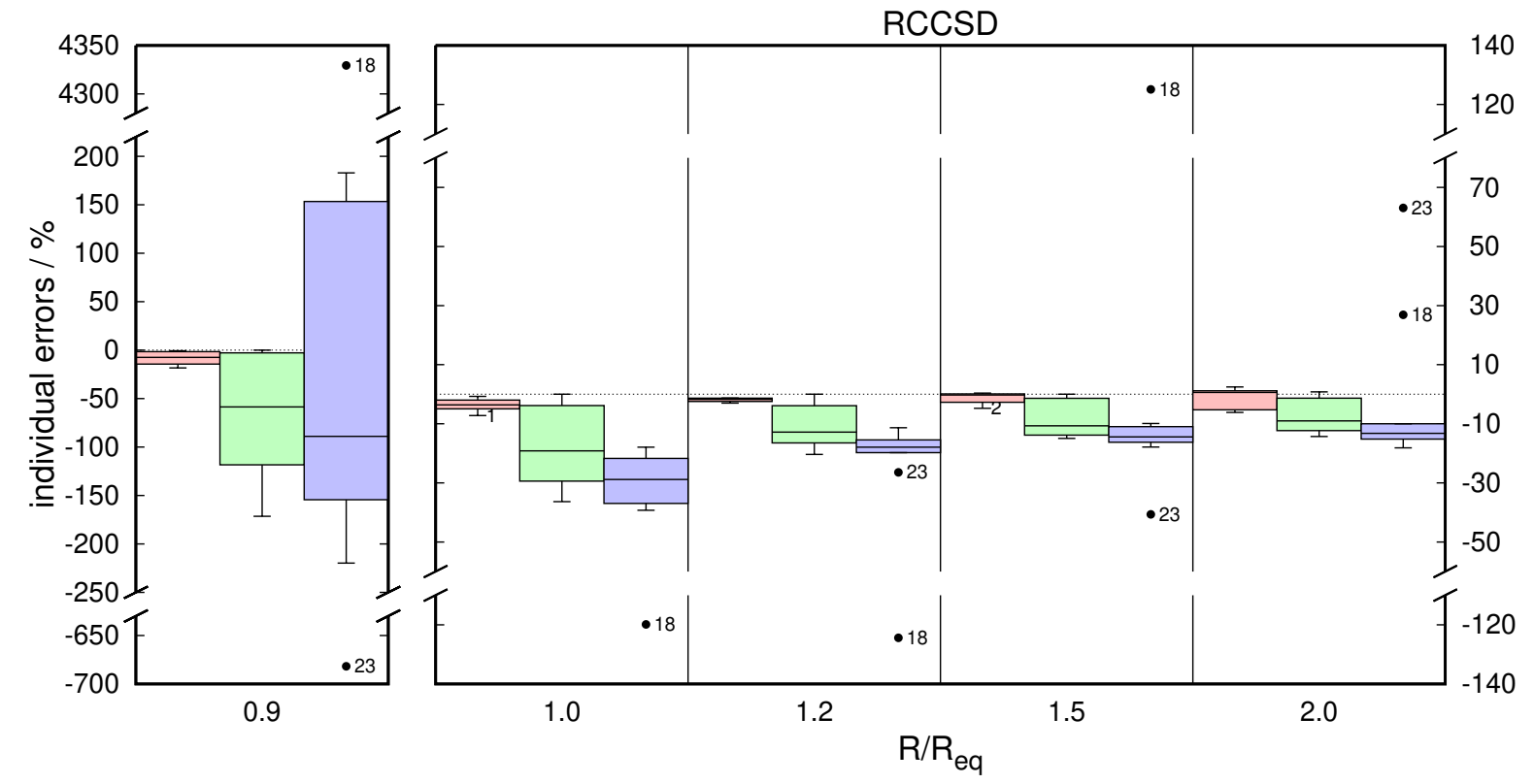

Figure S23: Box plot of signed relative errors (SRE, in percent) in the interaction energies at the RCCSD level of theory for the O24x5 data set divided in the electrostatic (ELST), mixed (MIX) and dispersion (DISP) subsets. The box and outer fences encompass $50 \%$ and $95 \%$ of the distribution, respectively. Outliers are labeled according to the order of systems given in Tables S3-S12.
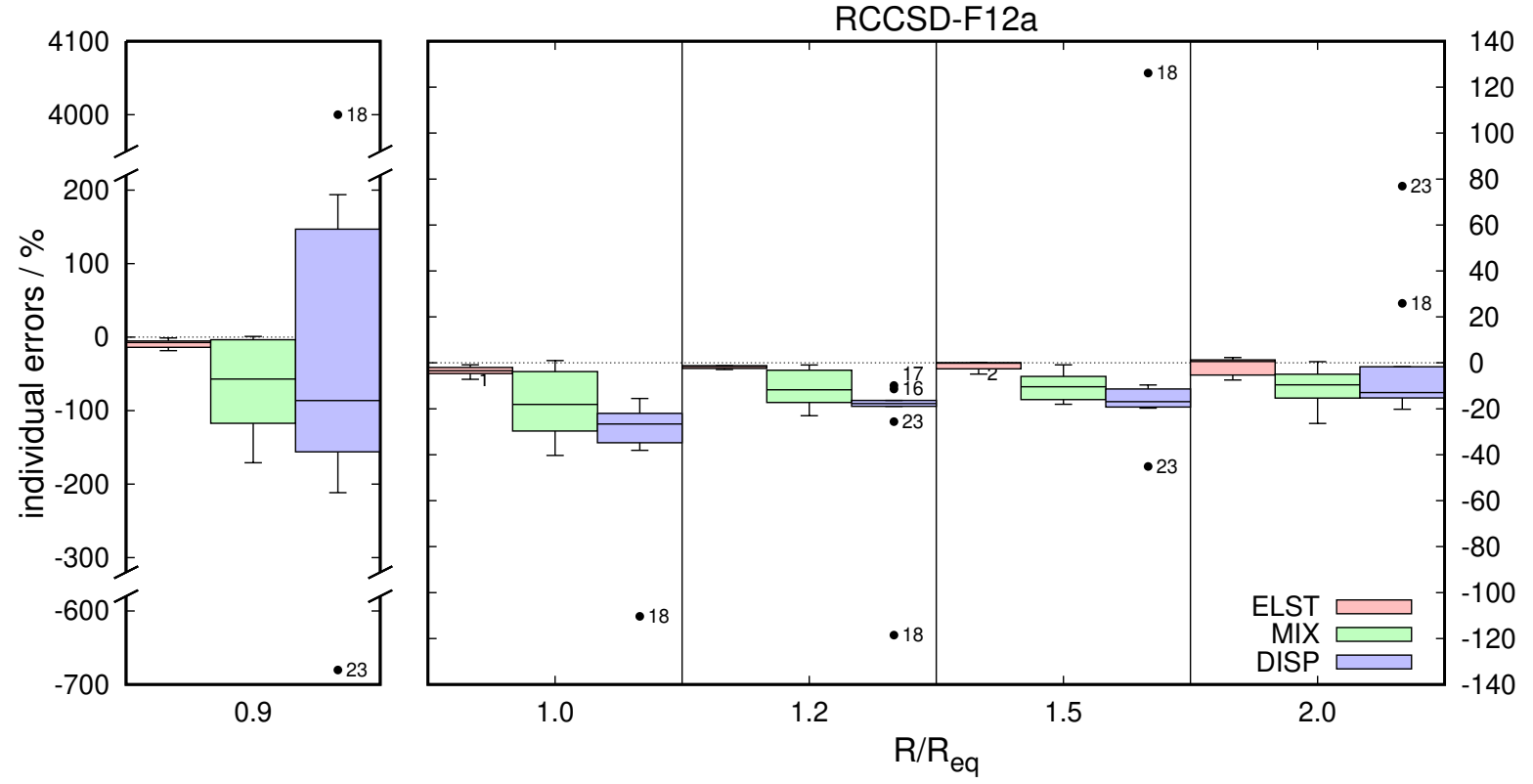

Figure S24: Box plot of signed relative errors (SRE, in percent) in the interaction energies at the RCCSD-F12a level of theory for the O24x5 data set divided in the electrostatic (ELST), mixed (MIX) and dispersion (DISP) subsets. The box and outer fences encompass $50 \%$ and $95 \%$ of the distribution, respectively. Outliers are labeled according to the order of systems given in Tables S3-S12. 

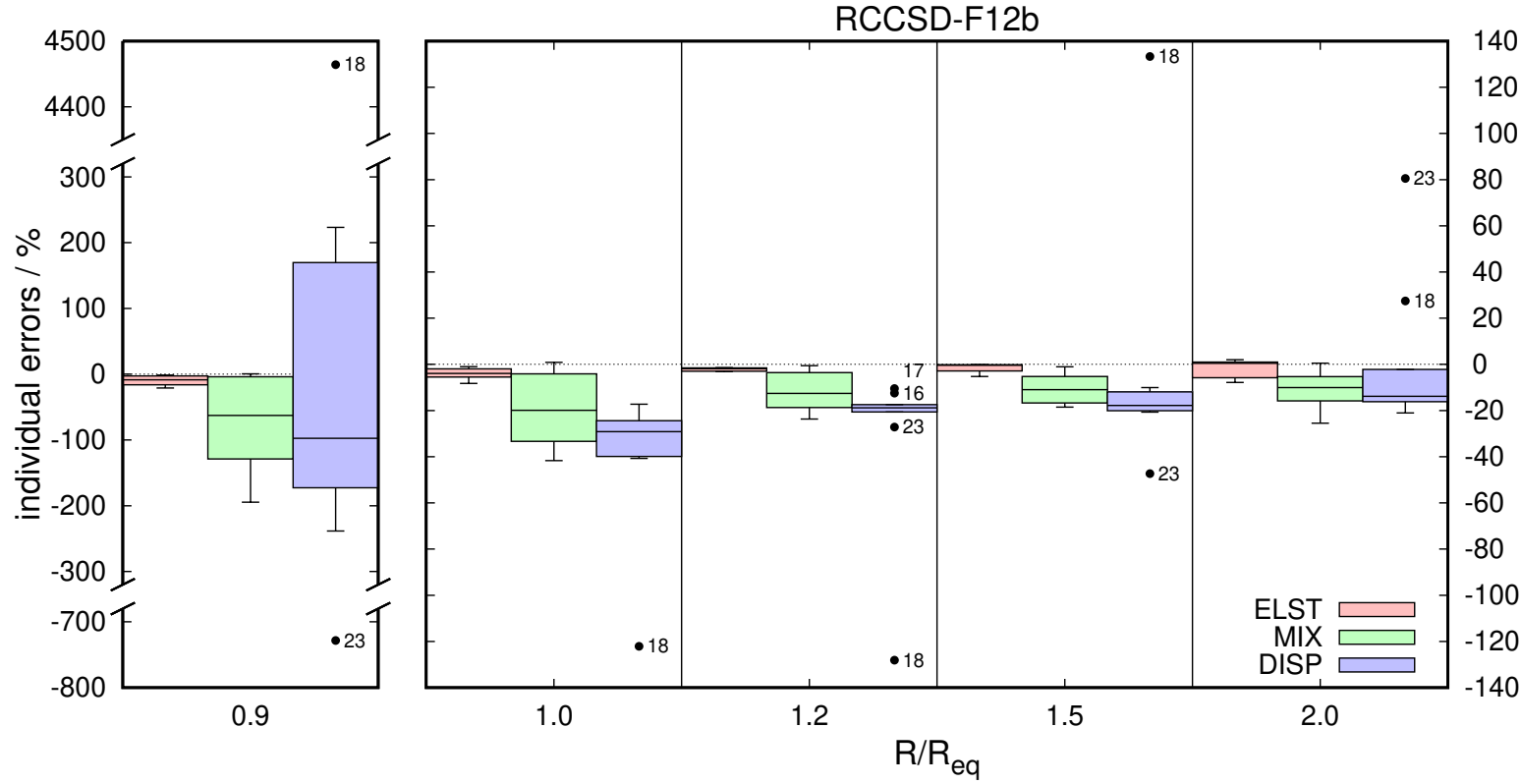

Figure S25: Box plot of signed relative errors (SRE, in percent) in the interaction energies at the RCCSD-F12b level of theory for the O24x5 data set divided in the electrostatic (ELST), mixed (MIX) and dispersion (DISP) subsets. The box and outer fences encompass $50 \%$ and $95 \%$ of the distribution, respectively. Outliers are labeled according to the order of systems given in Tables S3-S12.
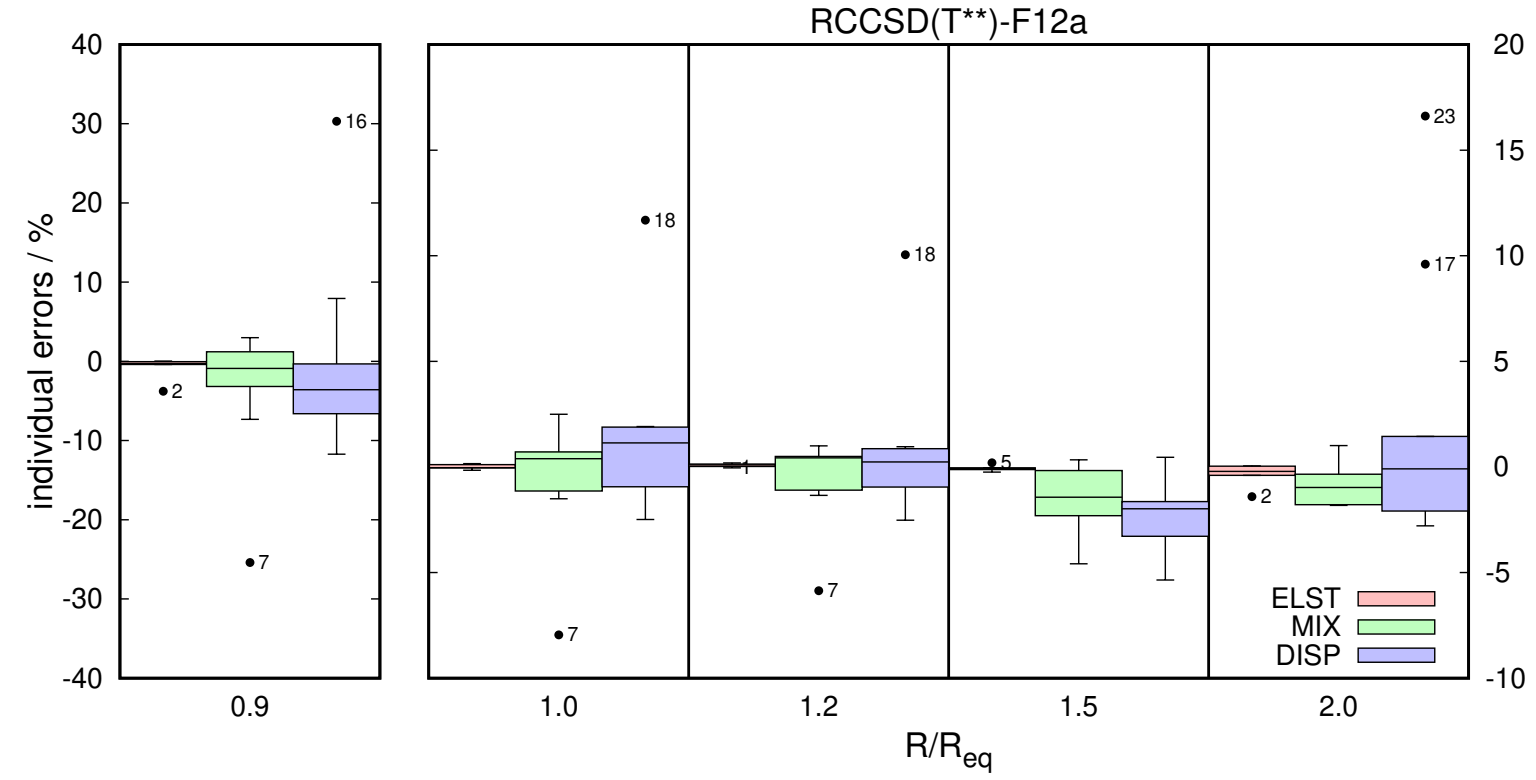

Figure S26: Box plot of signed relative errors (SRE, in percent) in the interaction energies at the $\mathrm{RCCSD}\left(\mathrm{T}^{* *}\right)$-F12a level of theory for the $\mathrm{O} 24 \mathrm{x} 5$ data set divided in the electrostatic (ELST), mixed (MIX) and dispersion (DISP) subsets. The box and outer fences encompass $50 \%$ and $95 \%$ of the distribution, respectively. Outliers are labeled according to the order of systems given in Tables S3-S12. 

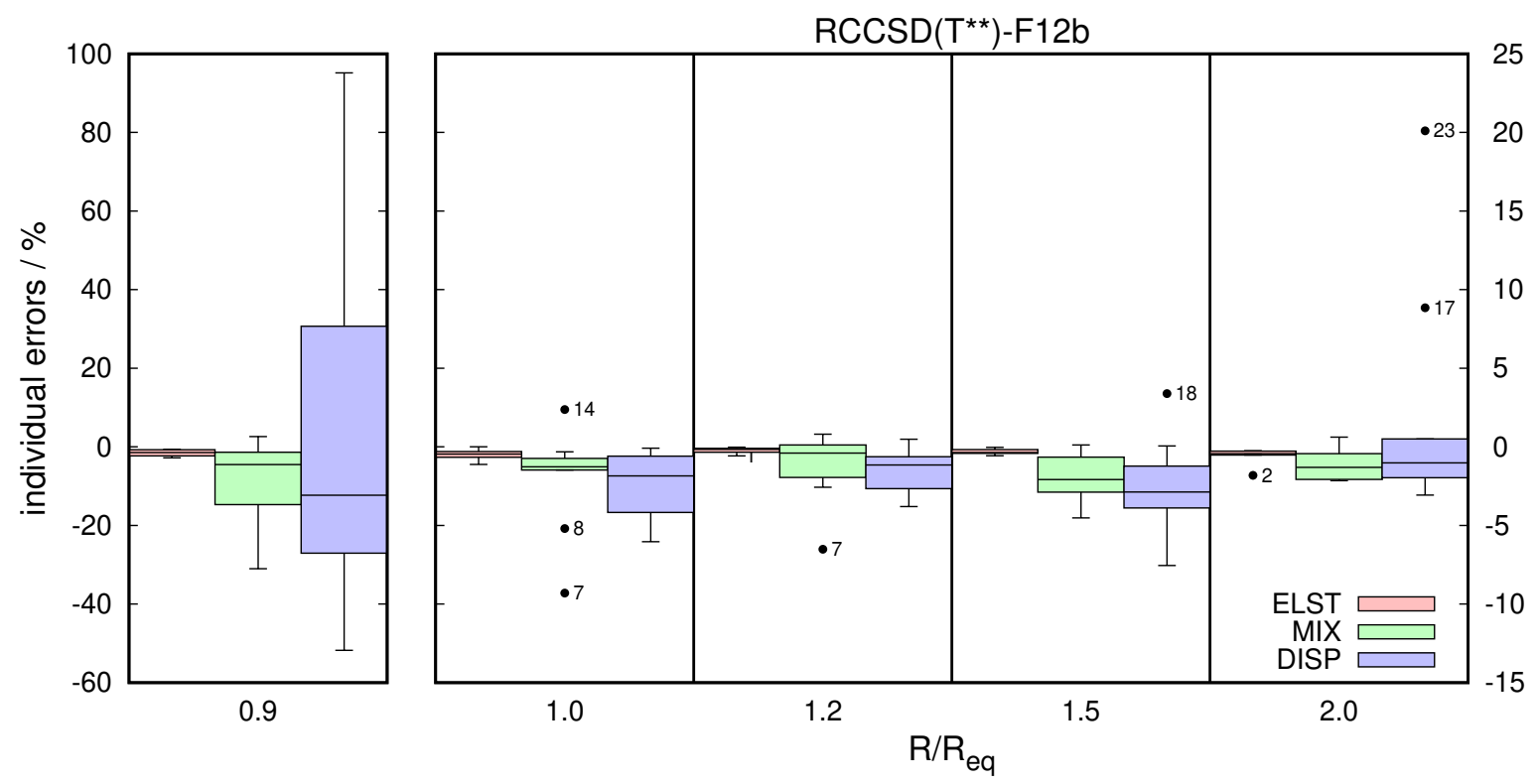

Figure S27: Box plot of signed relative errors (SRE, in percent) in the interaction energies at the $\mathrm{RCCSD}\left(\mathrm{T}^{* *}\right)$-F $12 \mathrm{~b}$ level of theory for the $\mathrm{O} 24 \mathrm{x} 5$ data set divided in the electrostatic (ELST), mixed (MIX) and dispersion (DISP) subsets. The box and outer fences encompass $50 \%$ and $95 \%$ of the distribution, respectively. Outliers are labeled according to the order of systems given in Tables S3-S12.

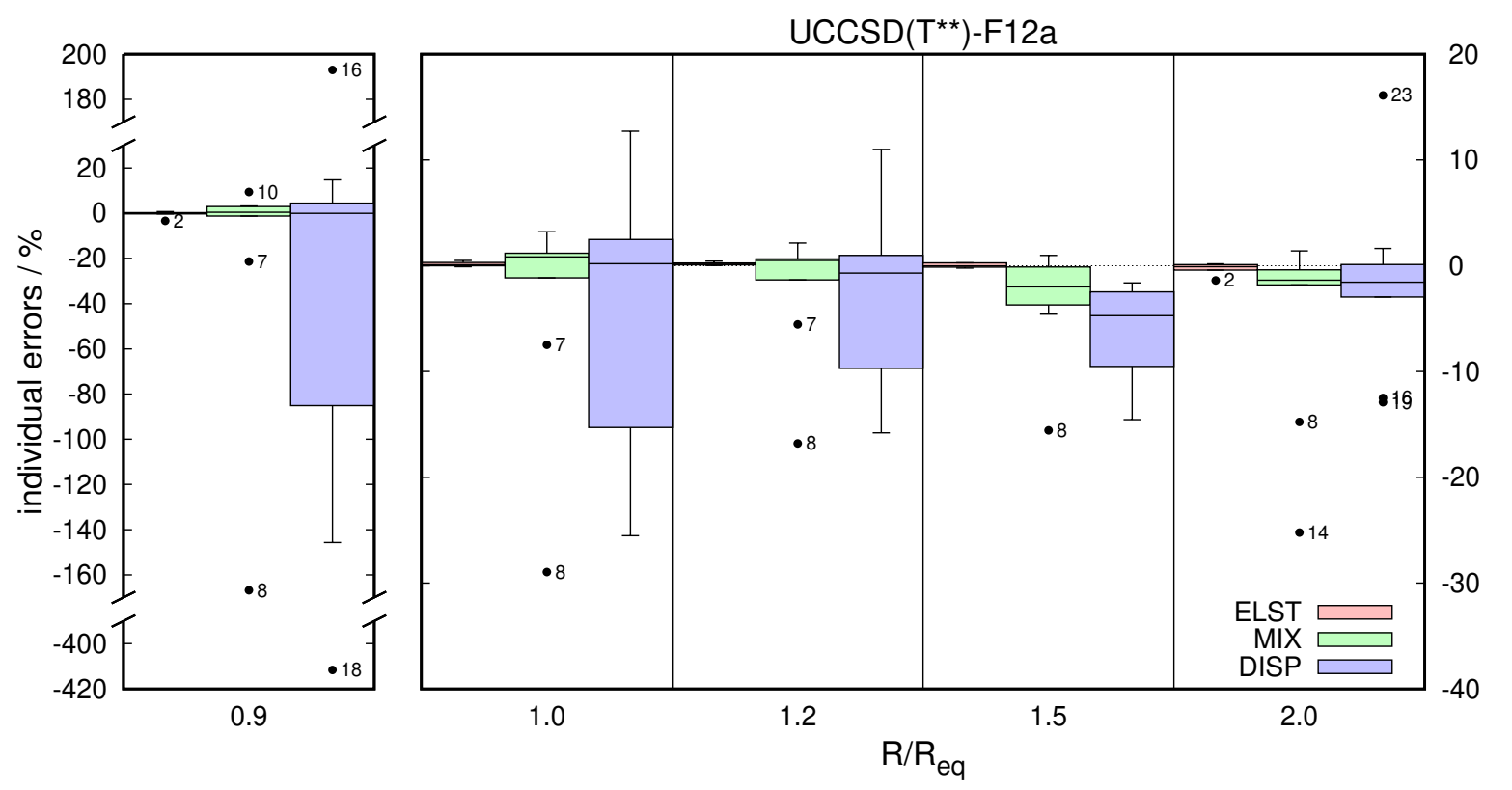

Figure S28: Box plot of signed relative errors (SRE, in percent) in the interaction energies at the $\mathrm{UCCSD}\left(\mathrm{T}^{* *}\right)$-F12a level of theory for the $\mathrm{O} 24 \mathrm{x} 5$ data set divided in the electrostatic (ELST), mixed (MIX) and dispersion (DISP) subsets. The box and outer fences encompass $50 \%$ and $95 \%$ of the distribution, respectively. Outliers are labeled according to the order of systems given in Tables S3-S12. 


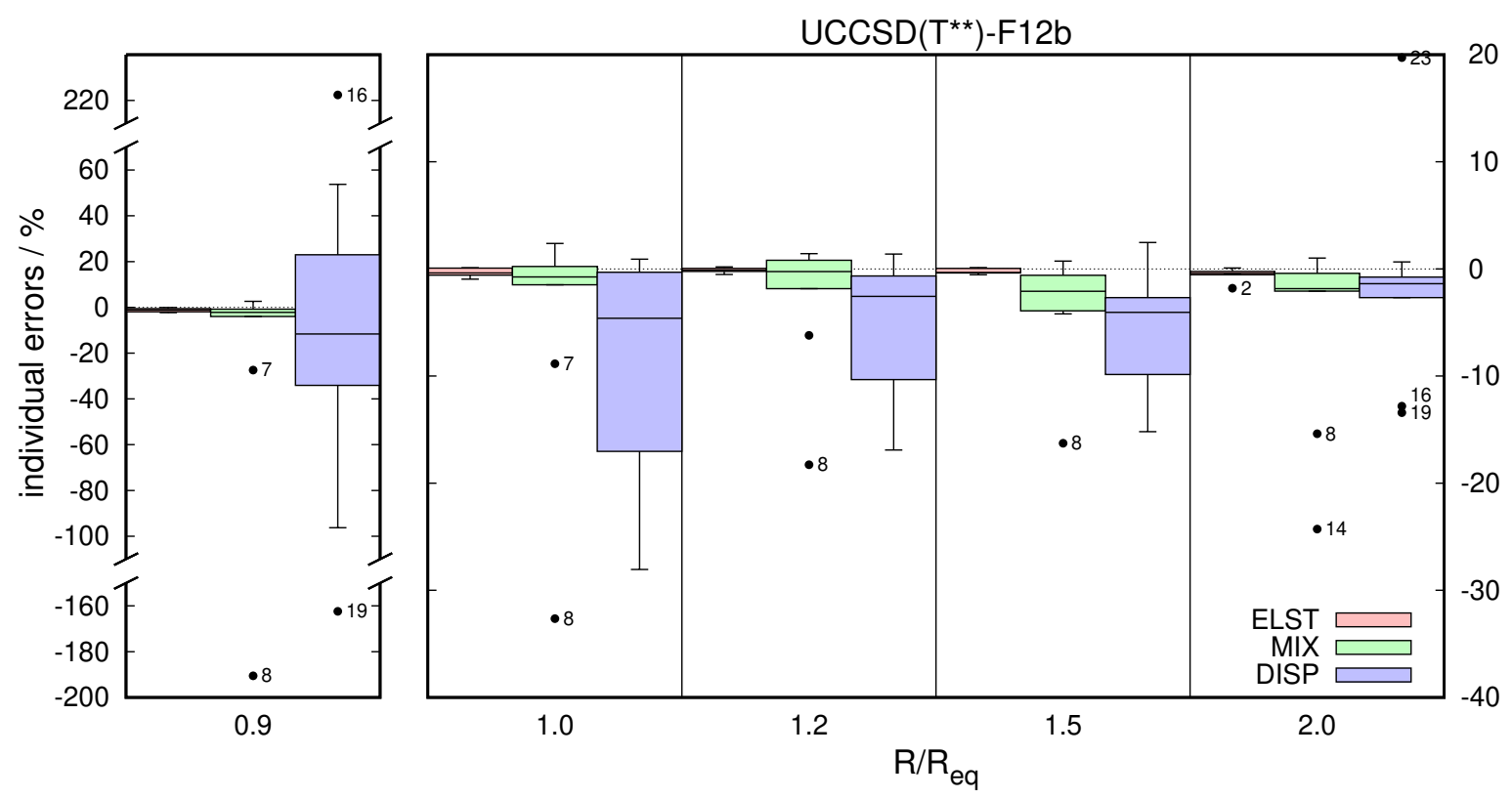

Figure S29: Box plot of signed relative errors (SRE, in percent) in the interaction energies at the $\mathrm{UCCSD}\left(\mathrm{T}^{* *}\right)-\mathrm{F} 12 \mathrm{~b}$ level of theory for the $\mathrm{O} 24 \mathrm{x} 5$ data set divided in the electrostatic (ELST), mixed (MIX) and dispersion (DISP) subsets. The box and outer fences encompass $50 \%$ and $95 \%$ of the distribution, respectively. Outliers are labeled according to the order of systems given in Tables S3-S12. 